
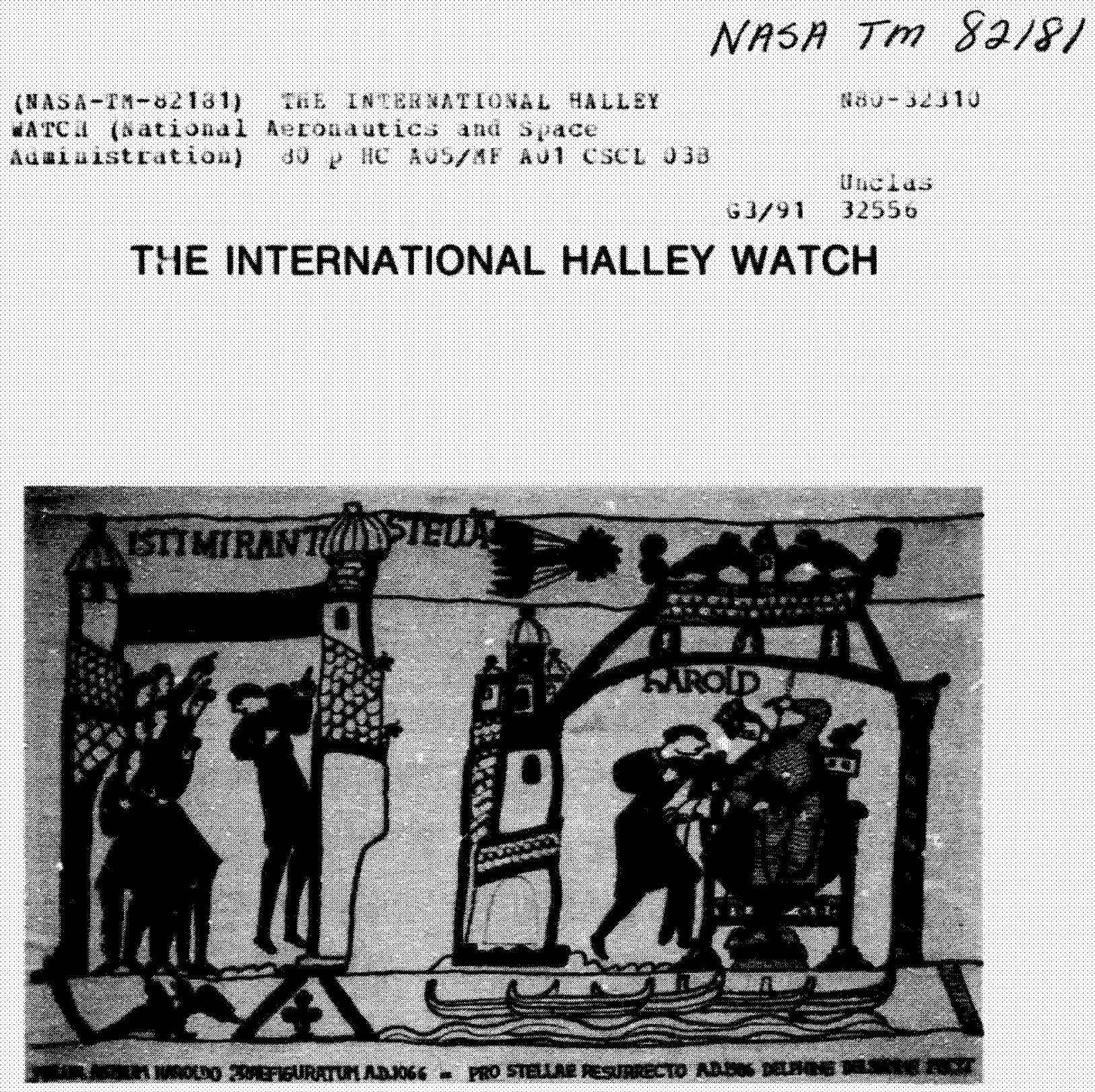

Report of the Science Working Group

July 1980

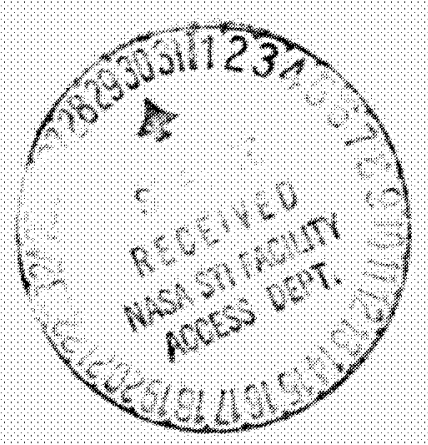




\title{
THE INTERNATIONAL HALLEY WATCH
}

\author{
Report of the Science Working Group
}




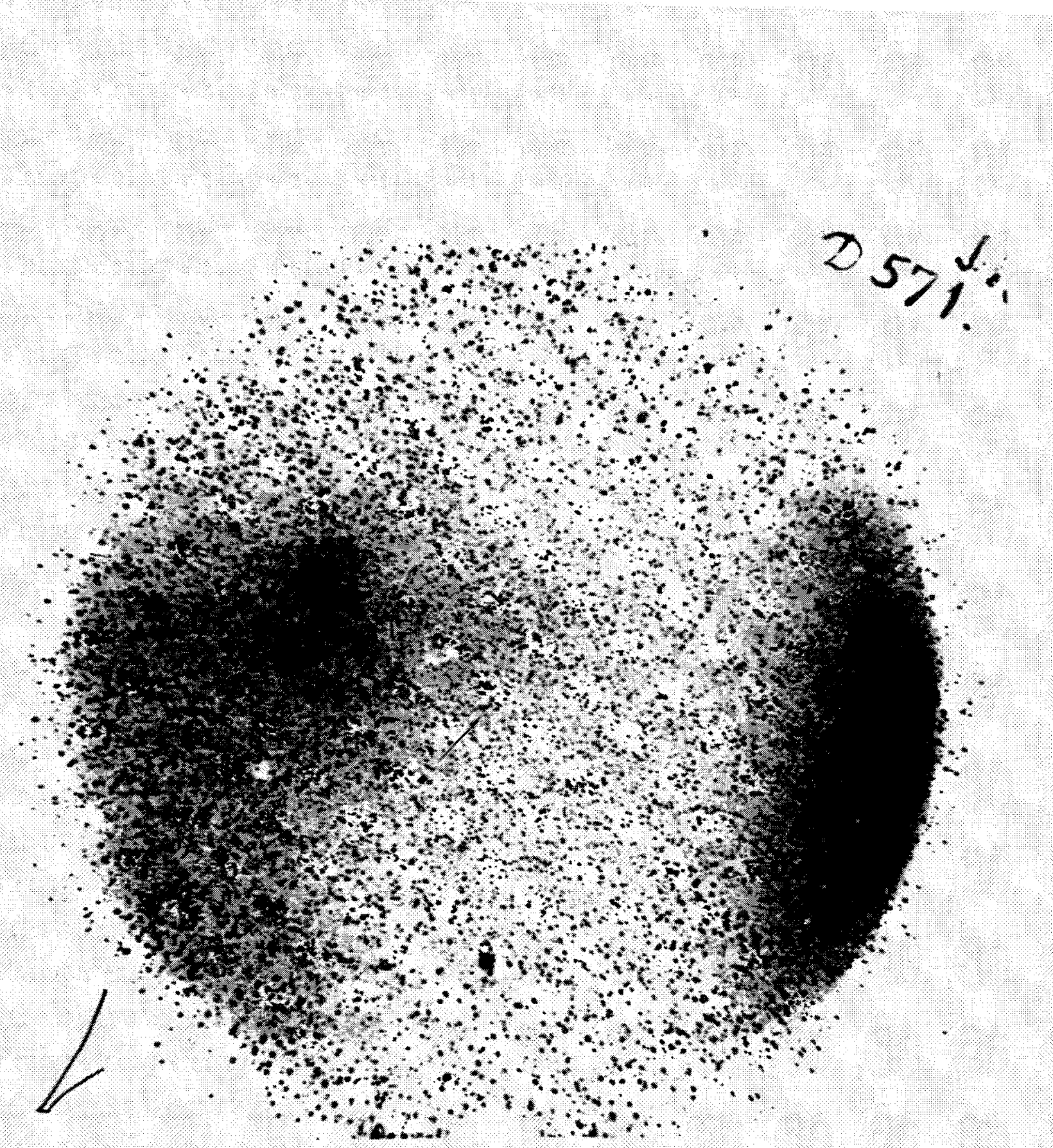

Conet Halley was discovered by Max Wolt at Heldel ber on Serternber II, 1909, near

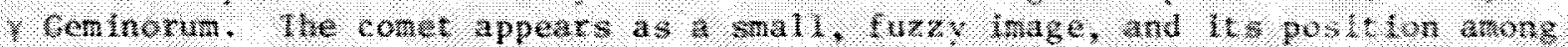
the star 19 marked. lt was stebsequently al so found on plates caken on lugust 24

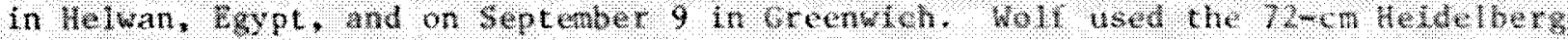

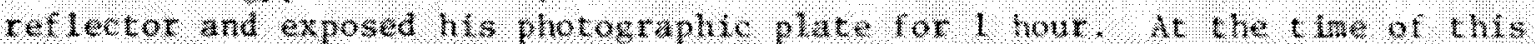

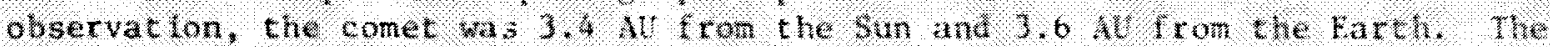

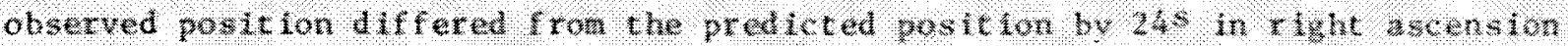
and $4^{*}$ in deslination. Halley's Cunet was deslighted $1909 \mathrm{c}$ and reached perluetion on aprel 20,1910 . 
1986 Apparition. Perihelion passage will occur on February 9, 1986, and attempts to recover the comet have already begun. Recovery is expected with some certainty no later than late 1983 and could be as early as late 1980. As of this writing, searches at the celestial position of Halley's Comet have established that it has not yet reached a brightness of $24.5^{\mathrm{m}}$.

\author{
No more I feel the potent spell \\ of Jupiter or Mars, \\ Or know the magic peace that fell \\ Upon me from the Stars, \\ A fiercer flame - a Comet Love \\ Consumes my spirit now; \\ I cry to you still heavens above, \\ "Oh! Halley's, where are thou?"
}

Punch May 11, 1910 


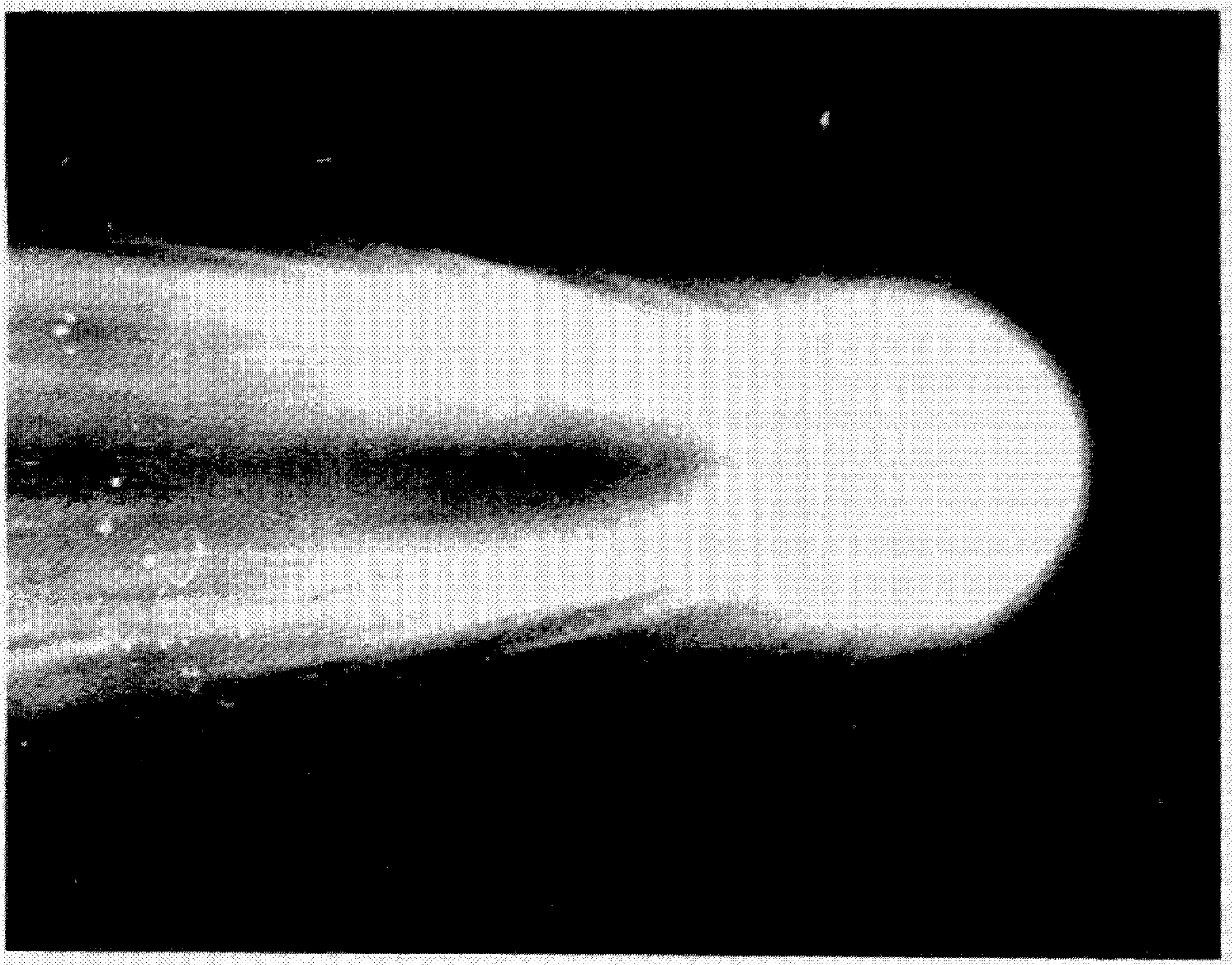

Head of HaMlley's Comet as seen on May 8,1910 (Wt. Wilson observatory photograph).

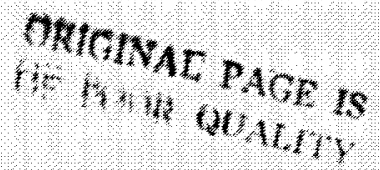




\section{CONTENTS}

The International Halley Watch Science Working Group ........ viii Sumnary and Conclusions .................. ix

I. Introduction ....................... I

II. Background ...................... 4

III. Synopsis of Comet Properties and Physics . . . . . . . . . 14

IV. Scientific Rationale for Studying Comets ........... 17

v. Why Study Comet Halley? ................... 18

vI. Scientific Objectives of Cometary Research in the 1980s . . . . 27

VII. International Halley Watch Purpose and Organization . . . . . 28

VIII. Interaction of the IHW with Observing Groups ........... 31

A. Deep Space Observations ................. 31

B. Earth Orbital observations ................ 31

C. Nonorbital Flight Techniques .............. 35

D. Earth-Based observations . . . . . . . . . . 37

IX. Ground-Based Observing Nets ............... 39

A. Large-Scale Phenomena .............. 39

B. Near-Nuc leus studies .................. 42

c. Spectroscopy ... . . . . . . . . . . . . 43

D. Photometry, Spectrophotometry and Polarimetry . . . . . 4 46

E. Radio Science ... . . . . . . . . . . . 49

F. Radiometry ................... 51

G. Astrometry .................. 53

References ..................... 55

Appendices

A Ephemeris for Comet Halley . . . . . . . . . . . . 56

B Observing Conditions for Comet Halley . . . . . . . . . 58

C Circular Respecting Observation of Halley's Comet - 1910 ..... 59 
THE INTERATIONAL BALEY WATCH

SCIENCE WORKING GROUP

Mchael F. A'Hearn

University of Maryland

Michael J. S. Belton

Kitt Peak National Observatory

John C. Brandt (Chairman)*

MASA-Goddard Space Flight Center

Geoffrey A. Briggs

NASA Headquarters

Donald E. Brownlee

University of Washington

William E. Brunk

MASA Headquarters

Mark R. Chartrand

American Museum, Hayden

Planetarium, N.Y.

Araand H. Delseme

University of Toledo

Donald L. DeVincenzi

NASA Headquarters

Bertram Donn

NASA-Goddard Space Flight Center

Chairman, IAU Commission 15

Louis D. Friedman*

(Manager, International Halley

Watch Study)

Jet Propulsion Laboratory

\author{
J. Michael Hollis \\ MASA-Goddard Space Flight Center \\ Ray L. Newburn (Vice-Chairman)* \\ Jet Propulsion Laboratory \\ Edward P. Ney \\ University of Minnesota \\ Jurgen Rahe \\ NASA-Goddard Space Flight \\ Cencer and University of \\ Erlangen-Nurnberg \\ Lewis E. Snyder \\ University of Illinois \\ Kurt Weiler \\ National Science Foundation \\ Edward J. Weiler \\ NASA Headquarters \\ Fred L. Whipple \\ Harvard University \\ Smithsonian Astrophysical \\ Observatory \\ Donald K. Yeomans* \\ (Executive Secretary) \\ Jet Propulsion Laboratory
}

JPL HALLEY WATCH STUDY TEAM

Jay T. Bergstralh

Louis D. Friedman
Ray. L. Newburn

Donald K. Yeomans

MEETINGS

March 24, 25, 1980

May $20,21,1980$

(Both meetings were held at the Goddard Space Flight Center)

*Editors for this report 
SUMARY AND CONCLUSIONS

The next decade will include the 1985-86 apparition of Halley's Comet, which for many reasons is a unique object. Scientifically, it is the only periodic comet that exhibits the full range of cometary phenomena, i.e., large coma, plasma and dust tails. Historically, it has been observed for over 2,000 years and has often been the source of great public interest as well as an object of curiosity, awe and fear. In addition, the orbit of Halley's Comet is well determined and hence its return can be predicted with confidence.

Preparaiion for such a major event (which occurs only every 76 years) will greatly enhance the scientific return and public appreciation of it. Therefore, a comprehensive progran to prepare for the next apparition has been initiated and it is called the International Halley Watch (IHW). The goals of the IHW are:

(1) To stimulate, encourage and coordinate scientific observations throughout the entire apparition.

(2) To help insure that observing techniques and instrumentation are standardized whenever possible.

(3) To help insure that the data and results are properly documented and archived.

(4) To receive and distribute data to participating scientists and to provide information to the public and media.

(5) To stimulate relevant instrument development where necessary.

The IHW will assume an advocacy role for the study of Halley's Comet and will provide liaison with facilities (missions, experiments, and observatories) outside the IHW organization itself for an active program of scientific measurements during the Halley apparition. For example, it will actively encourage observations of the comet from deep space and earth orbit. In situ measurements and near-comet observations from ballistic intercept missions will make an important contribution to the success of the IHW.

In addition, a major thrust will be the coordination of the ground-based observation effort. Possible observing nets include observers dedicated to the following subject areas and techniques: (1) large scale phenomena, (2) nearnucleus studies, (3) spectroscopy, (4) photometry, spectrophotonetry and polarimetry, (5) radio science, (6) radiometry, and (7) astrometry. The IHW plans to build on the experience gained at the 1909-1911 apparition of Halley's Comet by allocating suitable resources to the reduction, archiving, and publication of the observations. Finally, the 1985-86 return provides an opportunity to compare the properties of this bright, active and historic comet at two successive apparitions.

The Science Working Group for the IHW has met in the first half of 1930 and the Group strongly urges official formation and funding of the International Ha1ley Watch. 


\section{INTRODUCTION}

In early 1985, Comet Halley will cross the orbit of Jupiter and by the end of the same year the accelerating comet will have passed the asteroid belt, the orbit of Mars, and arrived again for its once-in-a-lifetime visit to the neighborhood of Earth. It is already obvious that this celestial event will be of enormous public interest. Ever since the ancient Chinese astronomers recorded their observations of Halley in $240 \mathrm{BC}$, it has been a source of curiosity, awe and fear. Swinging past the Sun only once every 76 years, the youthful comet has made the best of its brief moments near the Sun by showing off a bit - throwing out enormous amounts of gases and dust before returning to the frigid depths of space.

Unlike the last apparition in 1910 when the comet was easily seen, the coming 1985-1936 apparition will be unimpressive to the casual observer. The orbit projected on the plane of the ecliptic is shown in Figs. ! and 2, and a

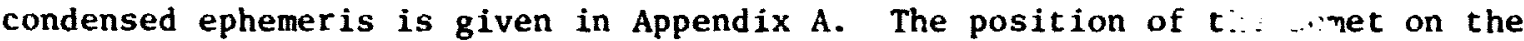
celestial sphere is shown in Fig. 3. The comet will be brightest during the first three weeks of February 1986, when it is nearest the Sun (closest approach occurs February 9). Unfortunately, the comet cannot be observed from the ground then because the Earth will be on the other side of the Sun. However, to observers who find a location away from city lighting and who are equipped with binoculars, Halley will be a rewarding sight in November and December of 1985 and at its best in March and early April of 1986. Those equipped with a telescope will witness an even more impressive display. Viewing conditions are summarized in Appendix B.

Although the appearance of Halley's comet in 1985-1986 will disappoint some of the waiting public, the scientific returns from this apparition will be enormous. Halley's comet is the oniy large comet displaying the full range of cometary activity that is predicted to return this century. Since it will not return again until 2061, the International Halley Watch should be organized to make the most of the coming 1986 opportunity in terms of science return and pub1ic information. 


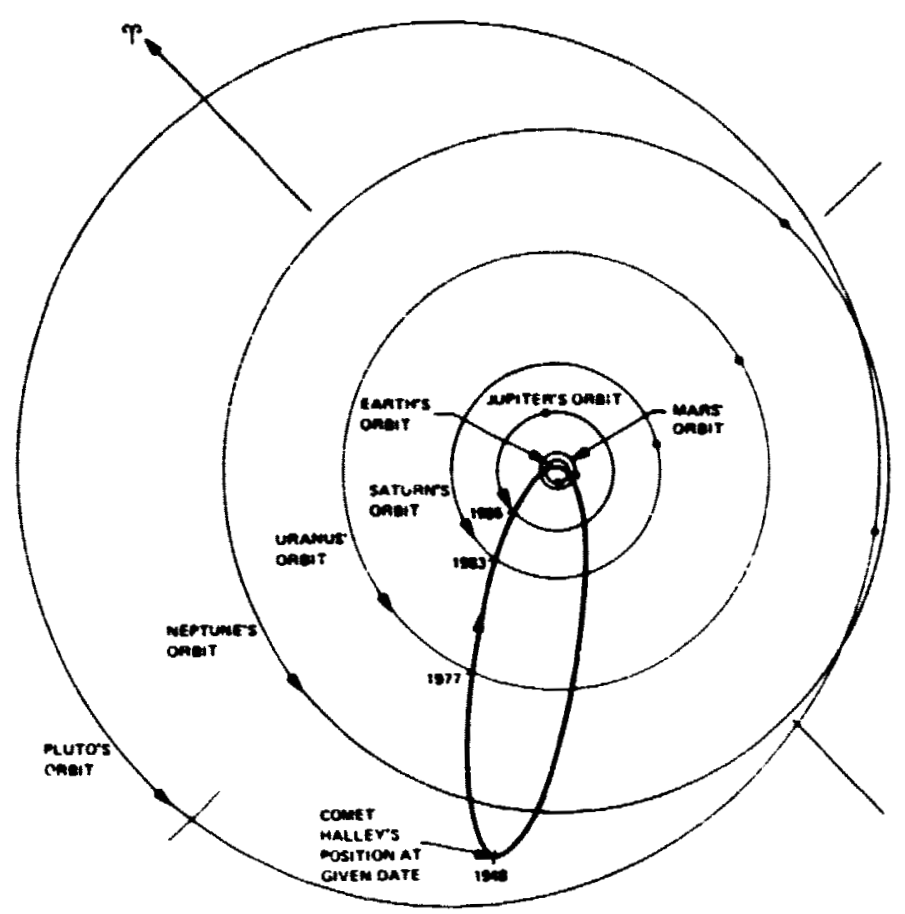

Fig. 1. Schematic drawing of Halley's orbit 1910-1986.

RELATIVE POSITIONS OF COMET HALLFY AND EARTH 1955-86

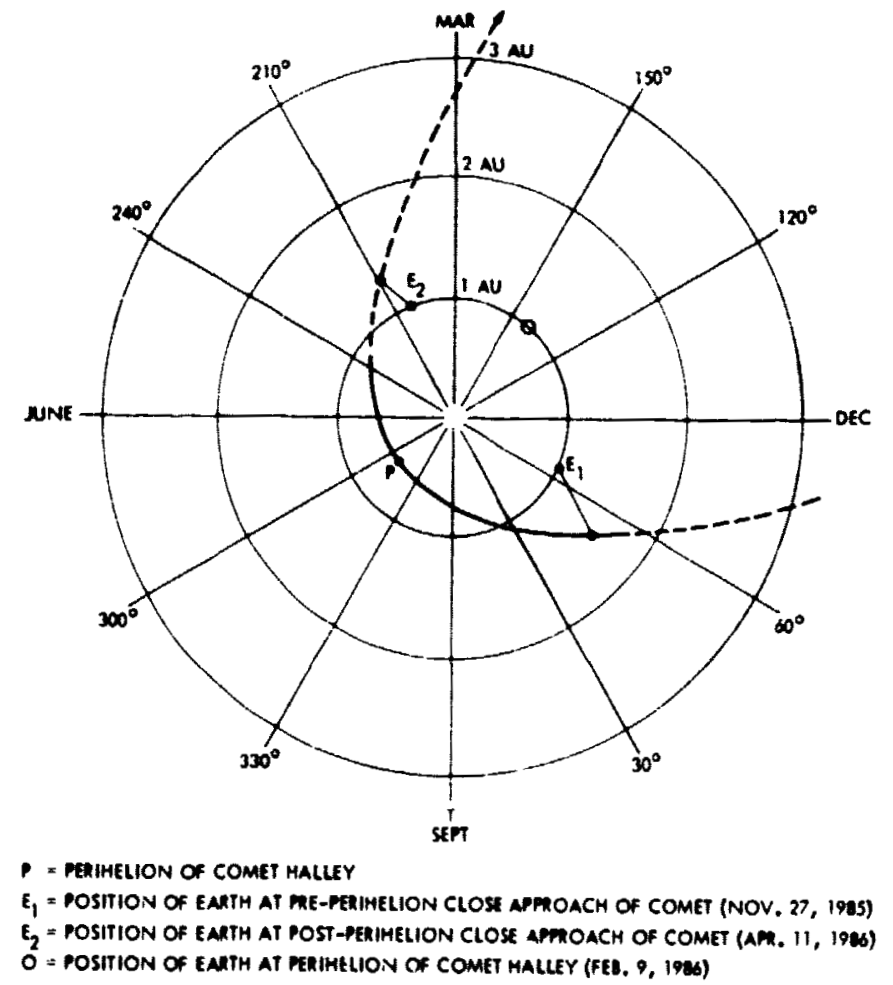

Fig. 2. Schematic drawing of Earth and Halley's orbit 1985-86. 


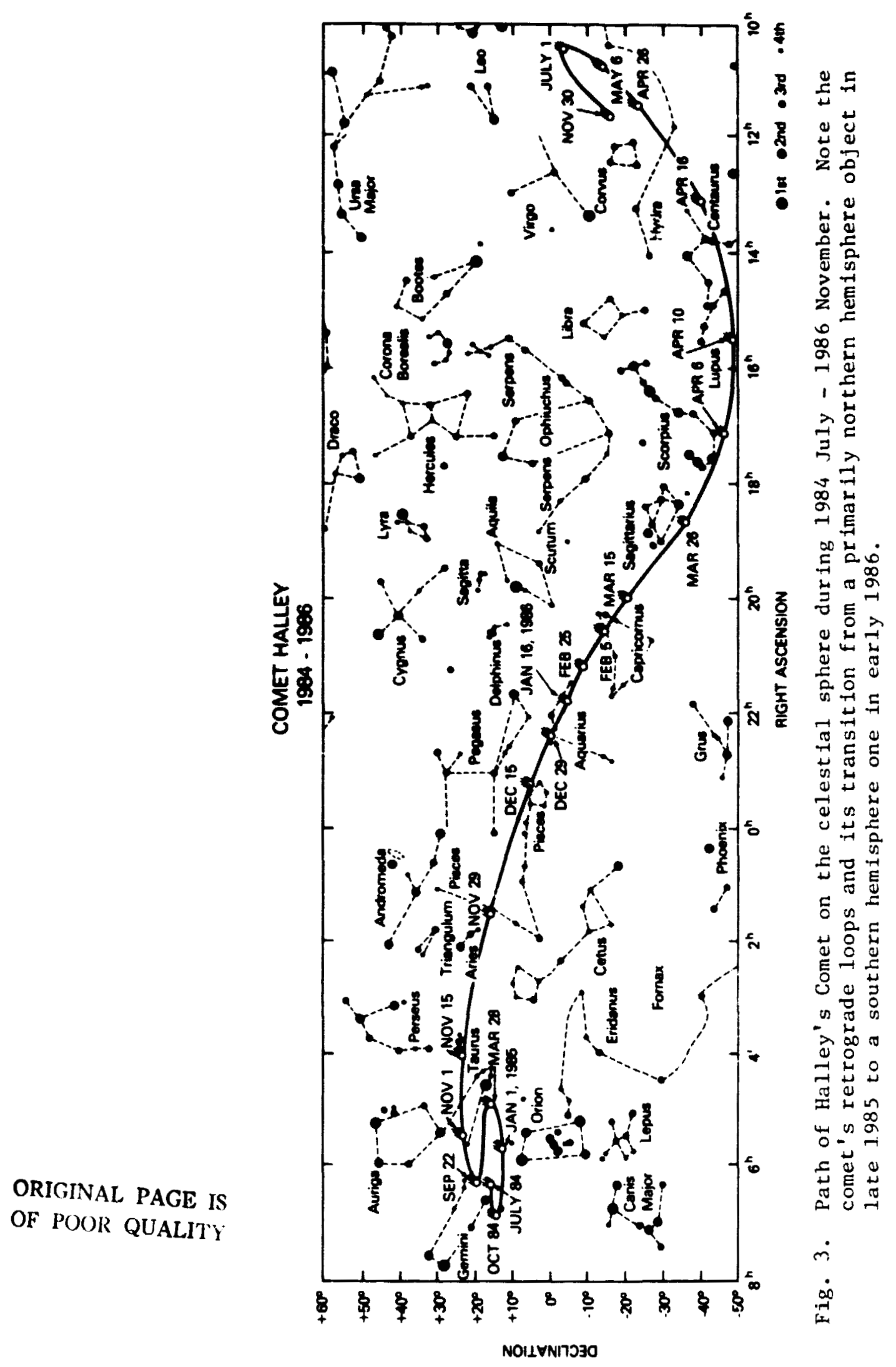




\section{BACKGROUND}

Scientific observations of Halley's Comet have been carried out for centuries (Fig. 4). In 1695 the English astronomer, Edmond Halley (Fig. 5) used positional data obtained during the apparitions observed in 1531, 1607, and 1682 to deduce that these sightings referred to the same comet. He published the work in 1705, predicting its return in 1758-1759. His prediction proved accurate.

In 1835, the comet was extensively observed by F. W. Bessel (Fig. 6). At this apparition the physical study of the comet was begun, but it became obvious that single observations provided only a snapsiot of the comet and that sequences were needed.

The scientific need for an organized network of coordinated observations in the study of a comet was suggested by E. E. Barnard as early as the 1890's, and just such a network was organized during the 1909-1910 apparition of Comet Halley (see Appendix C). The justification of that project was essentially identical to what it is today - to provide a continuous record of the comet from which to build physical models of observed phenomena. Although the 1909-1910 project had well defined goals and had established a central committee, the effort fell far short of its intended goals because many observatories did not cooperate with the central committee and because adequate funding and manpower were not available to use the enormous amount of data that was generated. Thus, in 1915 the Committee on Comets would report:

Subsequent developments have made it seem inexpedient to carry out the program above outlined. The photographs obtained at the Lick Observatory and at Cordoba are so numerous and excellent that they must have constituted a large part of the material reproduced and, since these observatories have indicated a purpose to reproduce their own photographs and a similar policy seems to be contemplated elsewhere, the Committee deems it unwise to undertake a duplicate publication, and equally unwise to make one from which this material is omitted.

It is important, as well as encouraging, to realize that the 1909-1910 project suffered from an inundation of useful data and not from a lack of it. An example of a photographic sequence obtained as a result of the network organization by Barnard is shown in Fig. 7. The plans for the collection of data were successfully carried out, but the data analysis and necessary publications were deficient. The only comprehensive study dealing with the last apparition appeared in 1931 - 21 years after Comet Halley's perihelion passage.

Whereas the 1909-10 observations of Comet Halley consisted only of groundbased visual and photographic measurements, the 1985-86 observations will include observations from deep space, Earth orbit, and ground observatories, covering the wavelength regions from the radio through the infrared and the visual to the ultraviolet. Planning, careful organization, and well-coordinated observations will be required to optimize the scientific return from comet Halley's brief visit. 


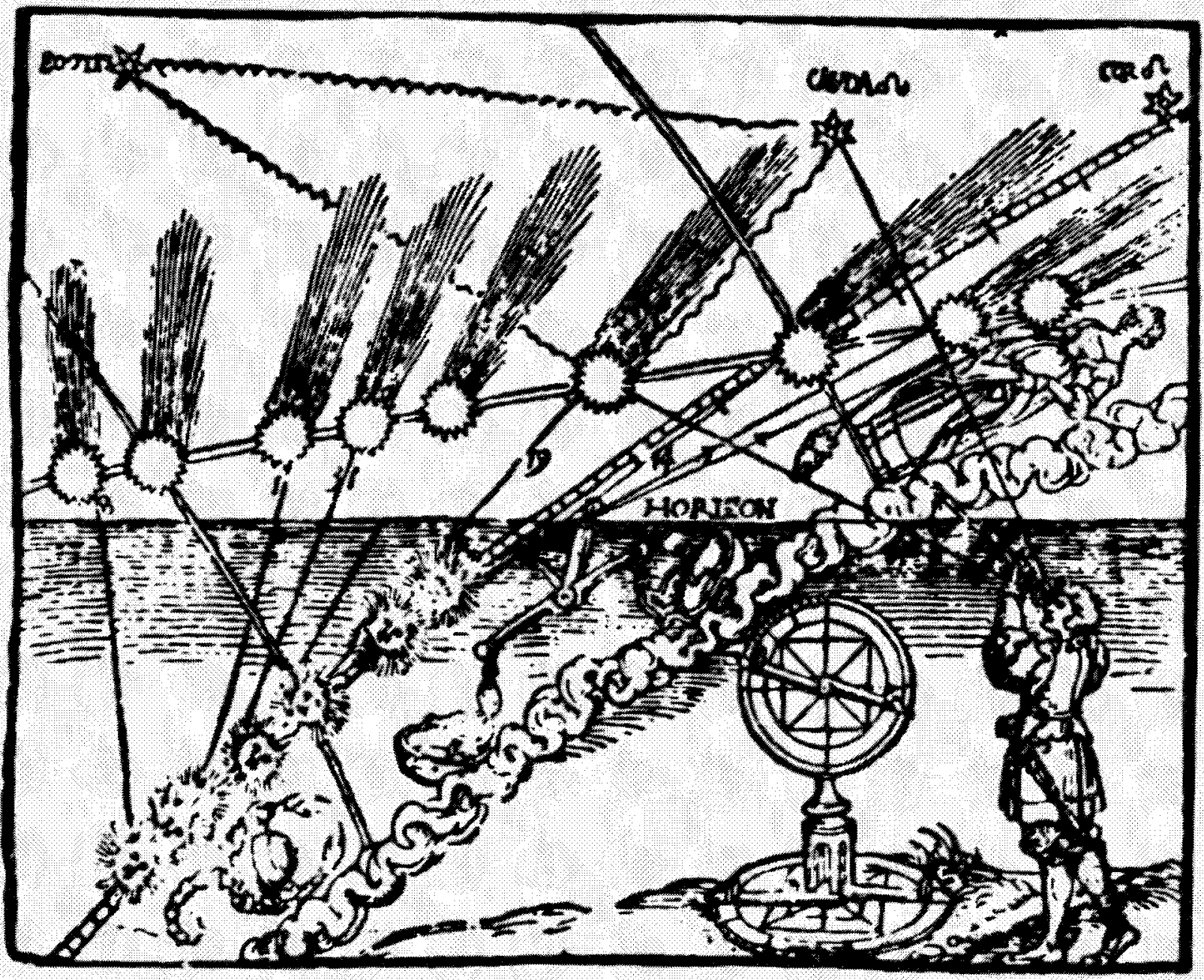

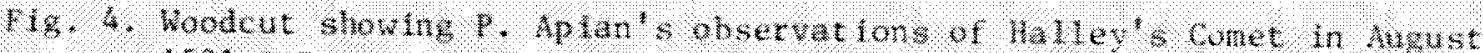
$1531,1.0$.

ORLWVW PWh is

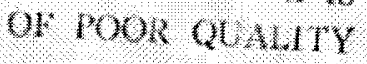




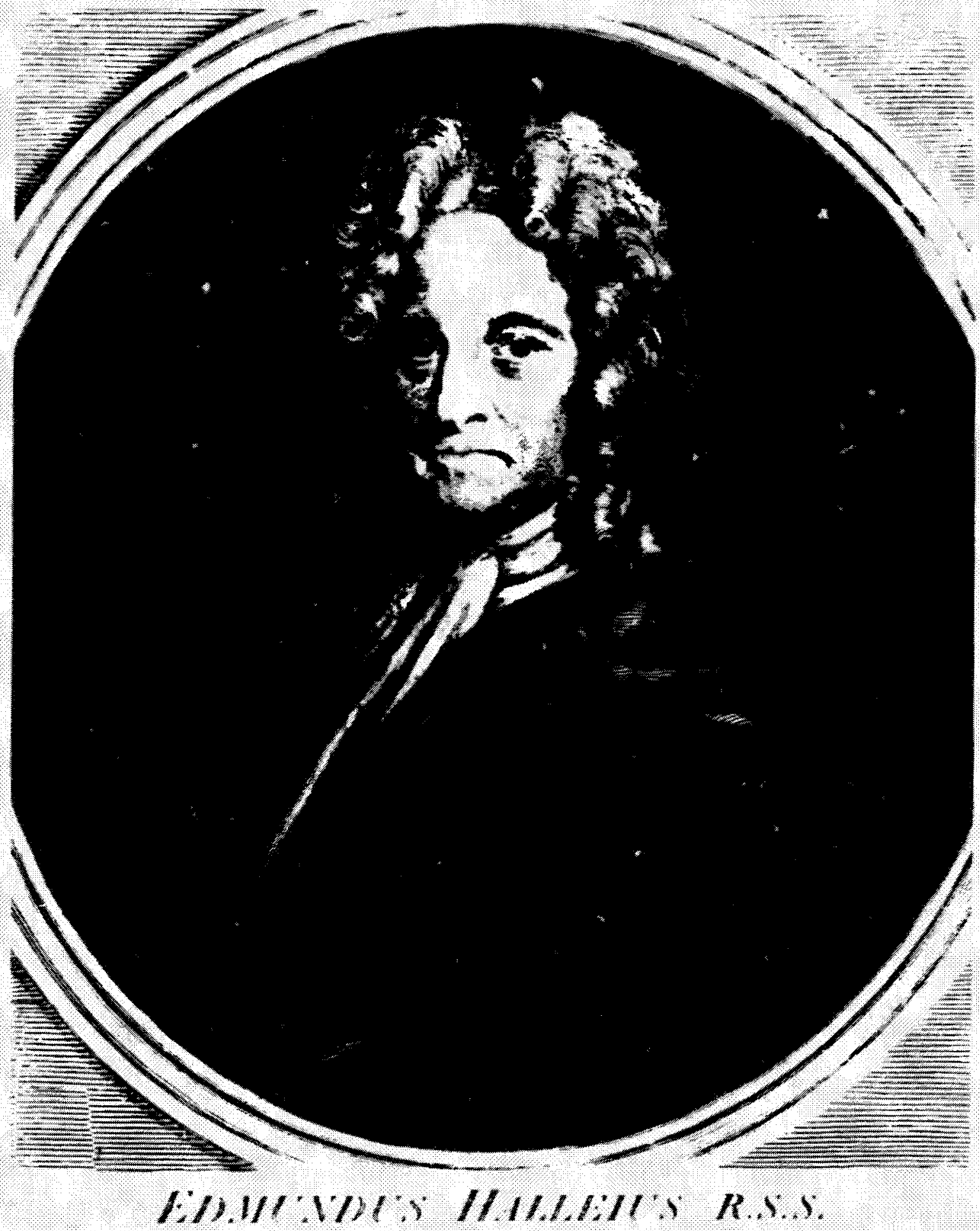

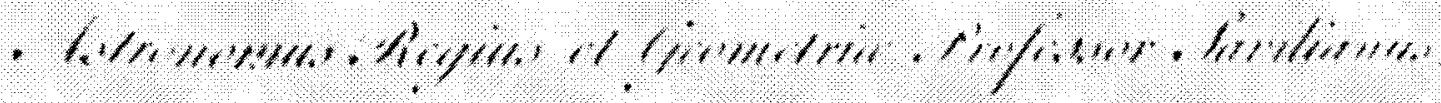

Fig. 5. Portrat of Edmond Halley $(1636-1742)$. 
ORICINM PACF is

wi pook QunimY

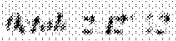

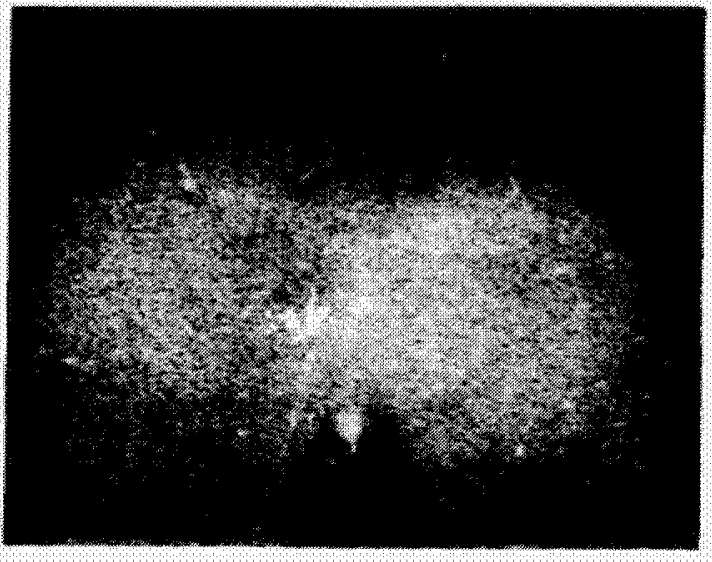

$0,1,1,6,5$

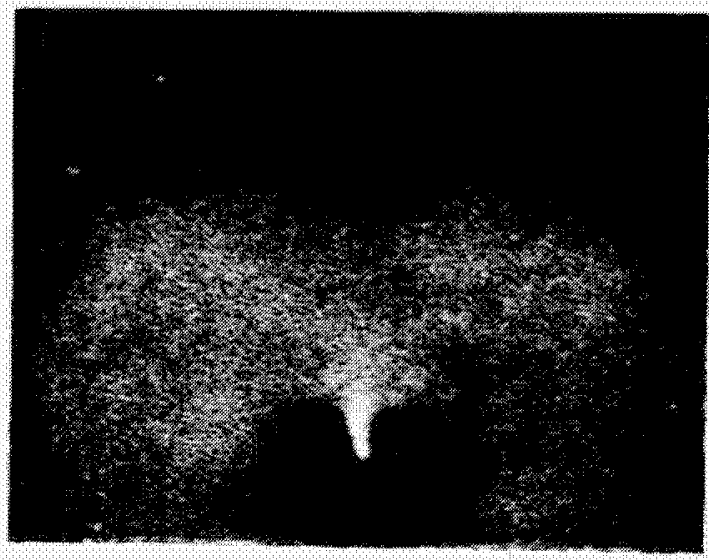

$0,01,0,0$

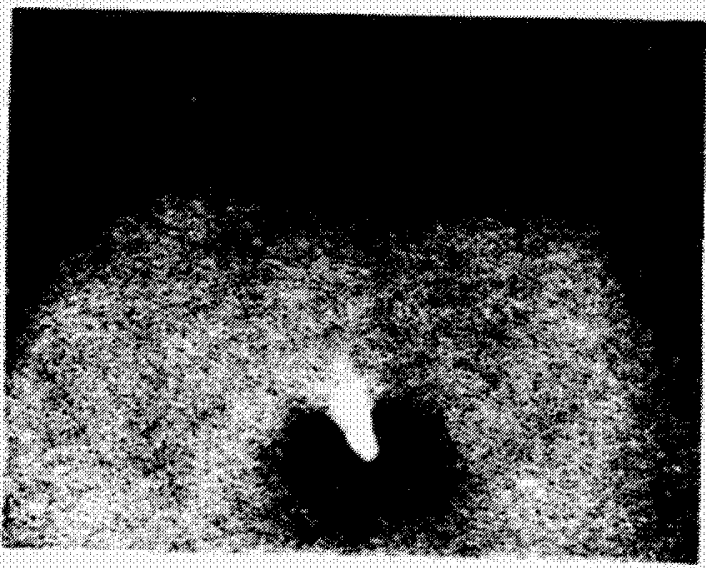

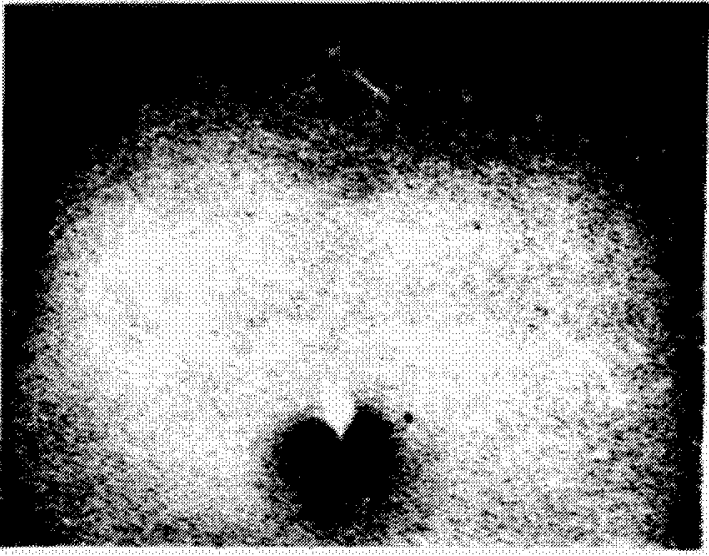

$a n+\infty$ ar $x$

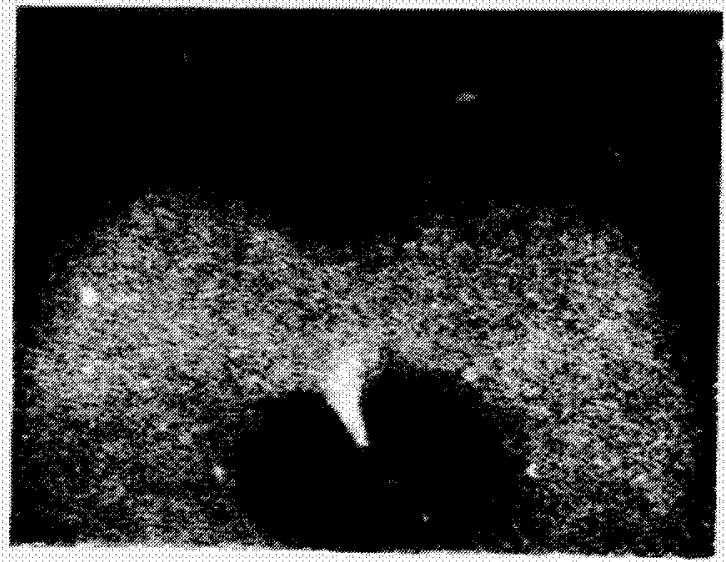

artan $2, \alpha$

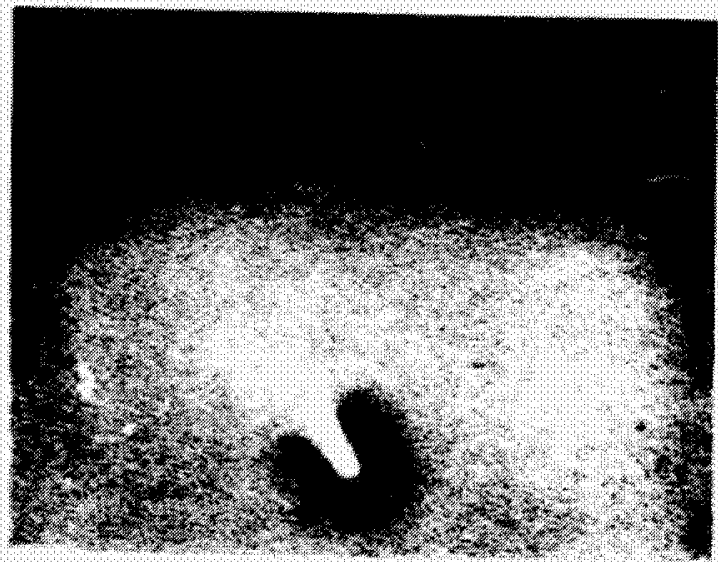

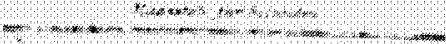

Fig. 6. F. W. Bessel's drawings of lialley's Comet at the 1835 appar ietion (after Astronomische Nachirichten, 13). 


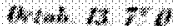

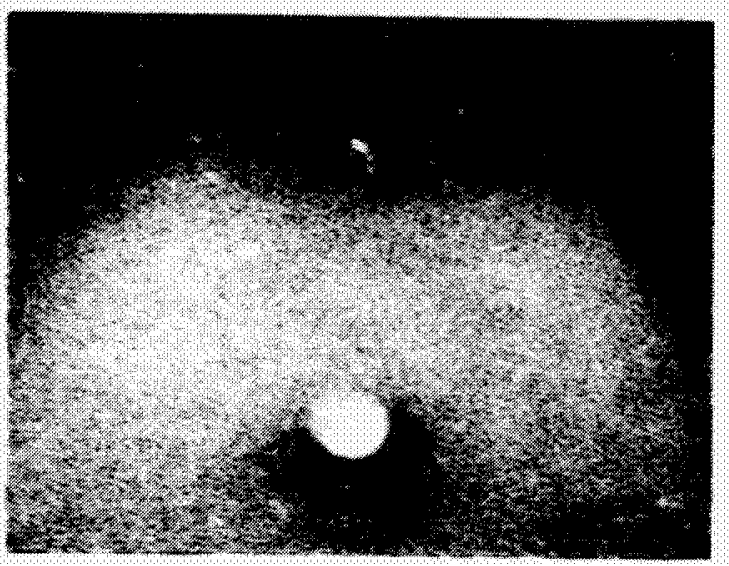

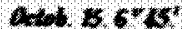

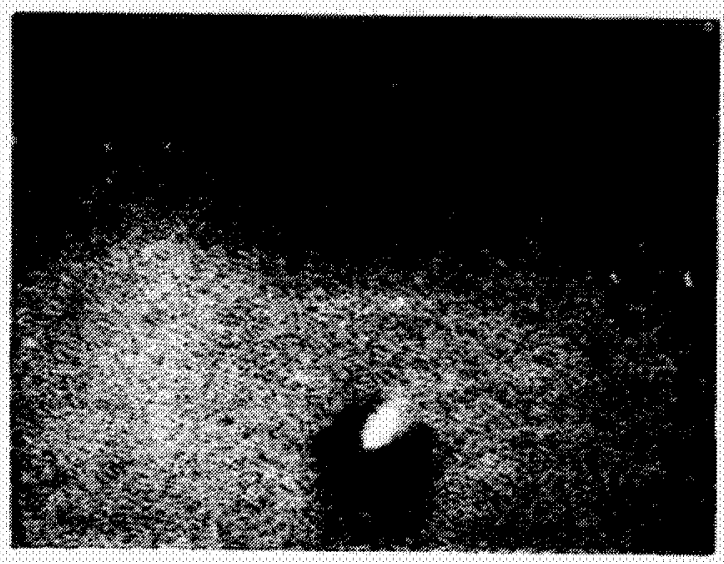

$0,0,24,6,3$

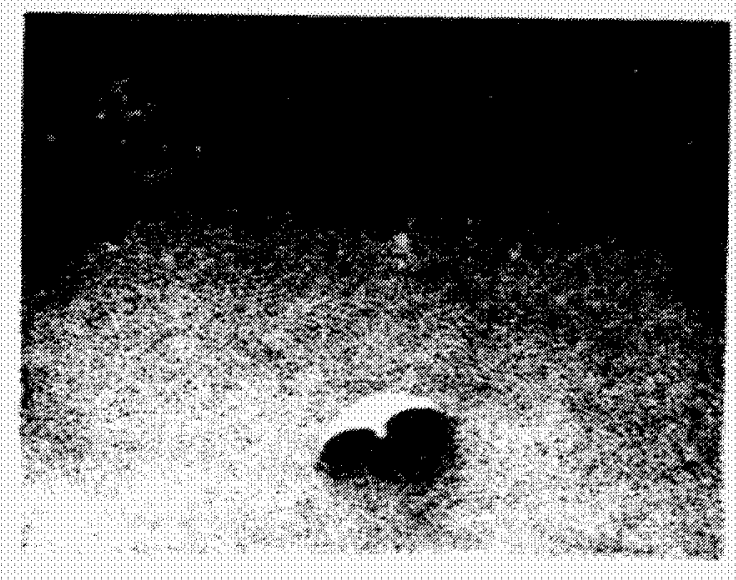

$n, w, w$

wn

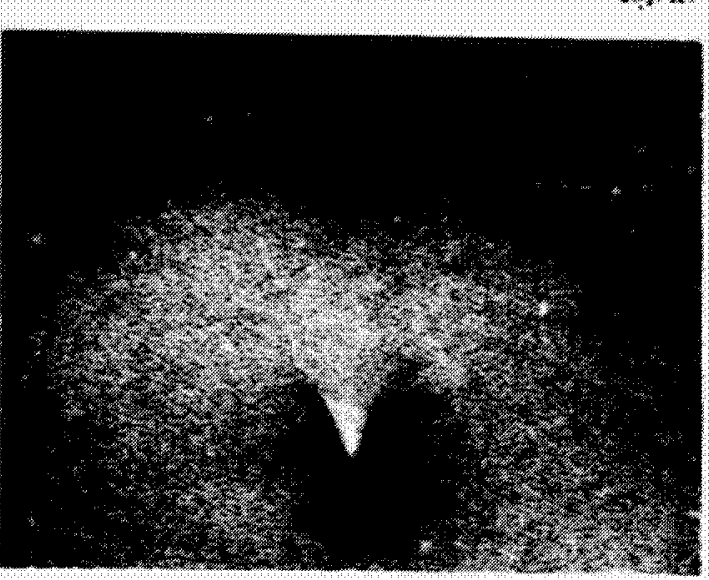

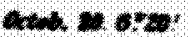

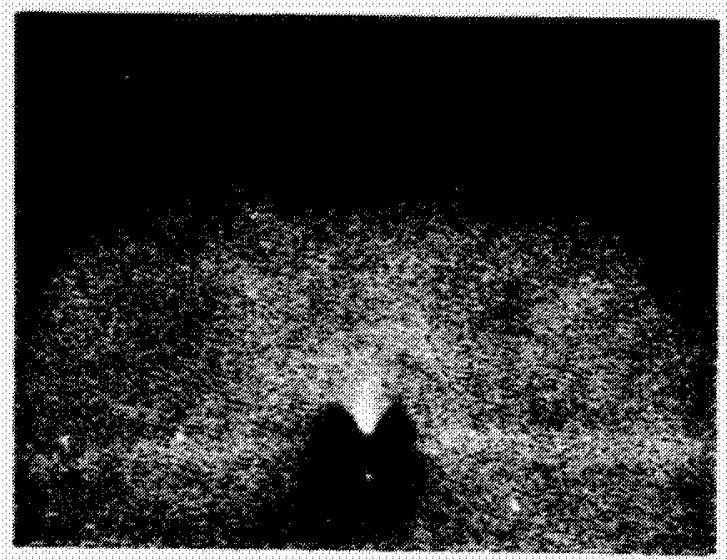

6, $, 0,6 \%$

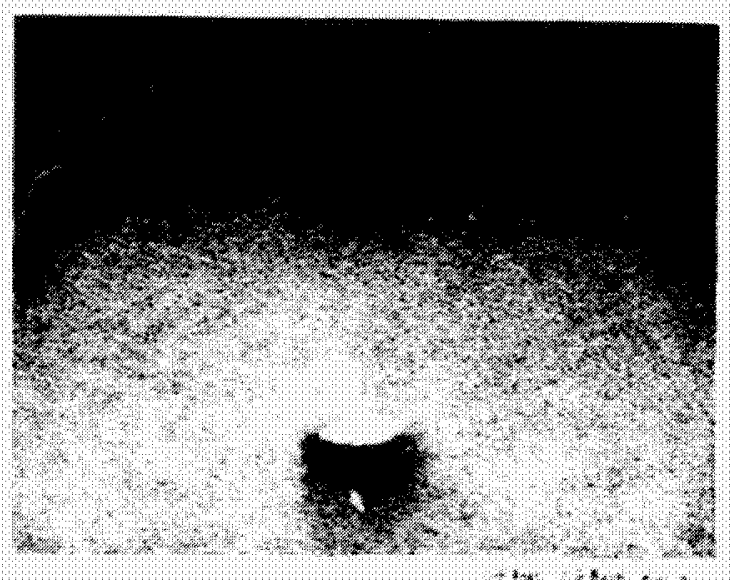

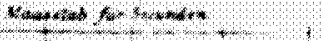

Hig. of (cont'd.) 

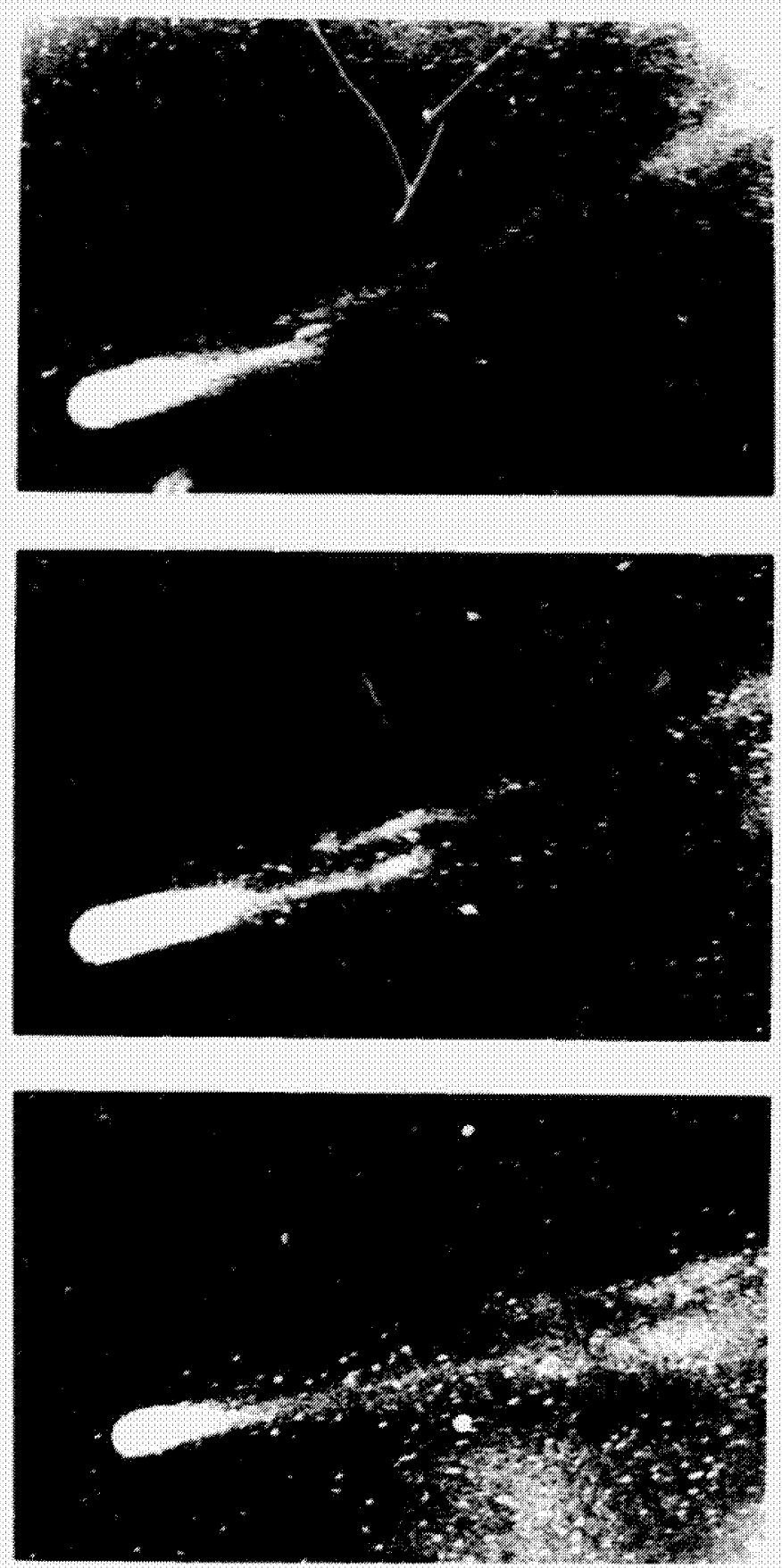

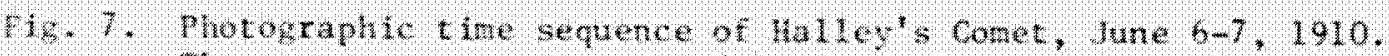
The sequence tas constructed by E. E. Barnard frota plates taken

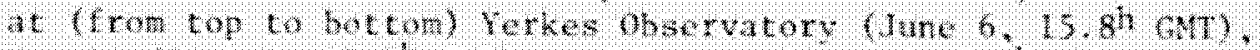

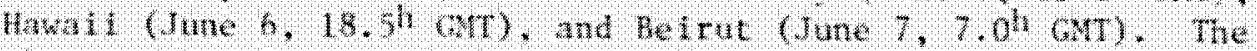
photographs ghow rapid changes in the form of a receding plasma Lall whell disconnected late on June 5 and whose thean recesslon

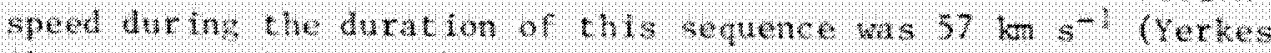
Observatory whotorathe. 
Besides the scientific history of Halley's Comet, there is a much longer record in human history of popular interest and association. A pictorial representation of Comet Halley in $684 \mathrm{AD}$ appears in the 1493 book entitled The Nuremberg Chronicles (Fig. 8), and the 1066 AD apparition is pictured on the Bayeux Tapestry (cover illustration).

During May of 1910, the tail of the comet was well developed and the view from Earth was spectacular (Fig. 9). On May 16, 1910, the Earth had an "encounter" with the tail; we either just missed it or passed through its outer fringes. To give an idea of the public excitement at that time, a collage of facsimile newspaper headlines is given in Fig. 10 .

It is abundantly clear that a complete program for the upcoming apparition of Halley's Comet must address the interests of the public and the mass media. This marvelous opportunity for scientific education of the general public must be included in the planning. 


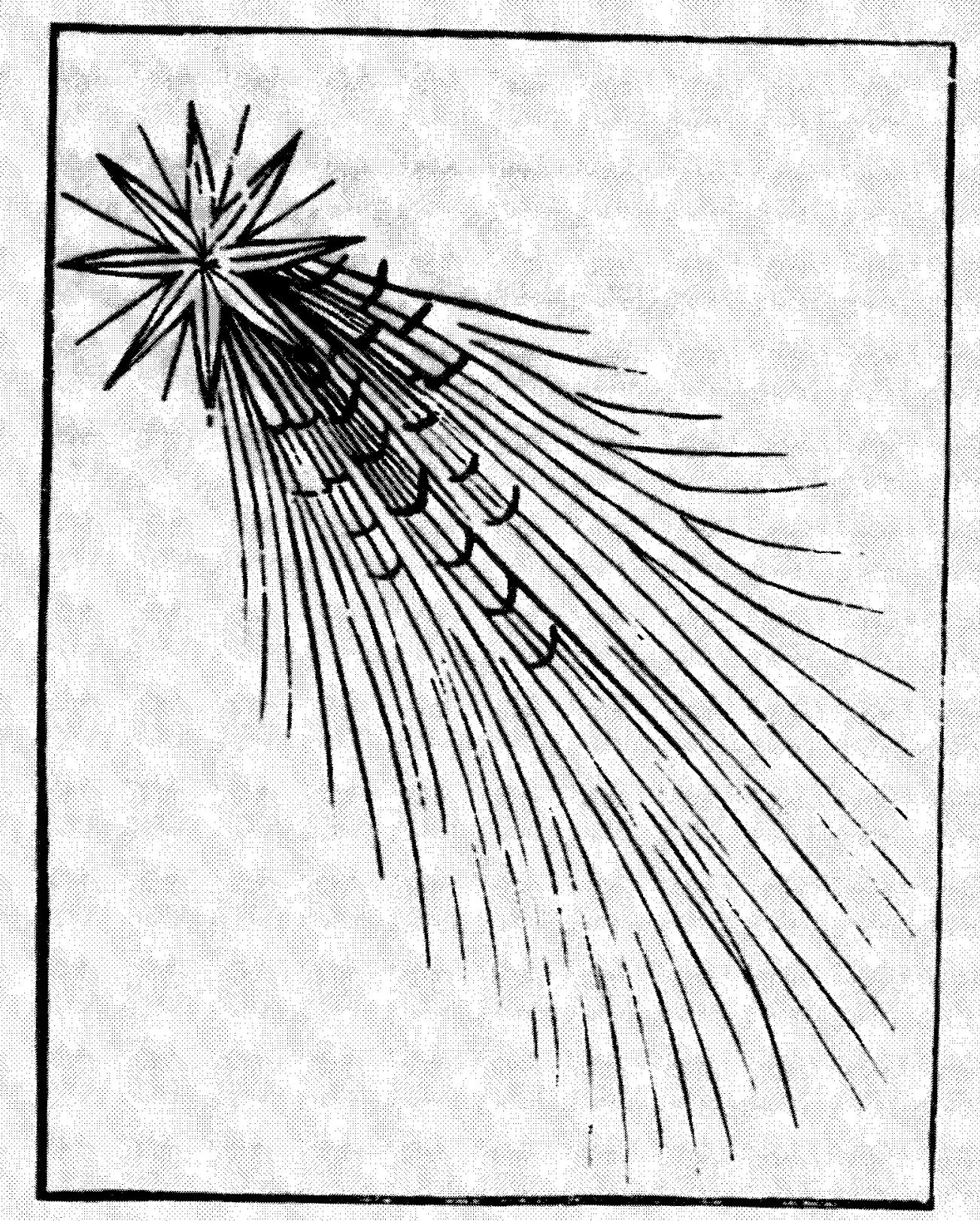

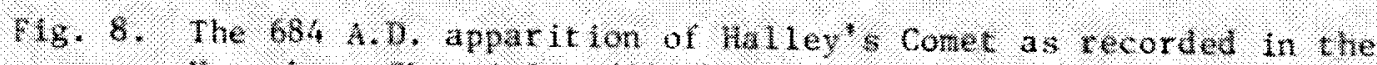
virevberg (wronicles (1493).

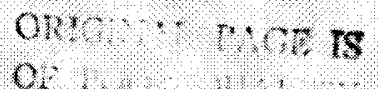

11 


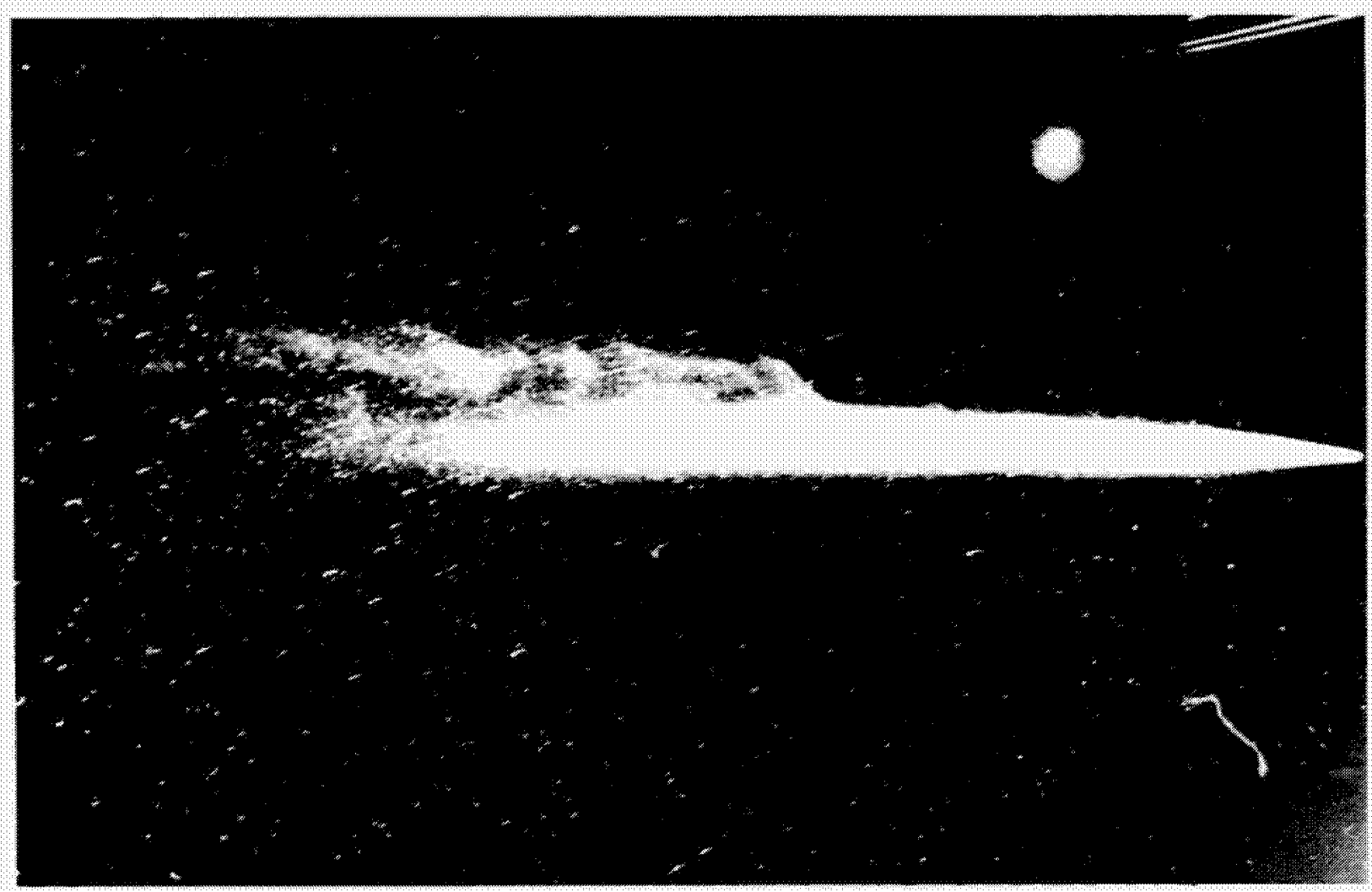

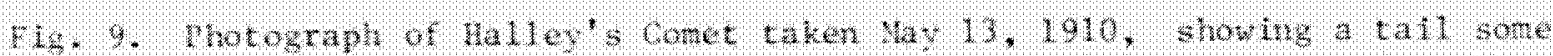
$45^{\circ}$ lims. The plasma tail is discommected and does not rum into the head reston; the angular geparation between the heat and the near end

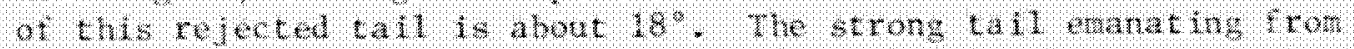
the cometars head is the dust $t$ all, and the bright object near che top of the (1. Id is Vents (Lowel) observatory photogranh). 


\section{BARNARD PICTURES OF HALLEY'S COMET}

Taken at Yerkes Observatory May 4, They Tally with Observation from Times Tower May 5.

VIEWED BY MISS PROCTOR

Negatives Show the Tail Extending

20 Degrees, Equivalent to $24,000,000$ Miles in Length.

\section{SIX HOURS TO-NIGHT IN THE COMET'S TAIL}

Few New Yorkers Likely to Know It by Ocular Demonstration, for It May Be Cloudy.

OUR MILLION-MILE JOURNEY

Takes Us Through 48 Trillion Cubic Miles of the Tail, Weighing All Told Half an Ounce!

\section{BALLOON TRIP TO VIEW COMET.}

\section{Aeronaut Harmon Invites College}

Deans to Join Him in Ascension.

\section{IN COMET'S TAIL ON WEDNESDAY}

\section{Eurepenen and Anmeriean Astremomers Agree the Earth Will Not Soffier in the Poranes.}

\section{TELL THE TIMES ABOUT IT}

And of Proposed ObservationsYerkes Observatory to Use Bal. loons if the Weather's Cloudy.

\section{TAIL 46,000,000 MILES LONG?}

Scarfed in a Filmy Bit of It, We'll Whirl On In Our Dance Through Space, Unharmed, and, Most of Us, Unheeding.

\section{MAY SEE COMET TO-DAY.}

Harvard Observers Think It May Be Visible in Afternoon.

Pror. Hall Doubts This, Though, but

There's No Danzer. Anyway.

\section{YERKES OBSERVATORY READY.}

$$
\begin{aligned}
& \text { Fxperts and a Battery of Cameras and } \\
& \text { Telescopes Already Prepared. } \\
& \text { CHICAGO IS TERRIFIED. } \\
& \text { Women Are Stopping Up Doors and } \\
& \text { Windows to Keep Out Cyanogen. }
\end{aligned}
$$

\section{CHICAGO IS TERRIFIED.}

\section{MAY BE METEORIC SHOWERS.}

Fig. 10. Facsimile newspaper headlines from the New York Times coverage of Halley's Comet in May 1910. 


\section{SYNOPSIS OF COMET PROPERTIES AND PHYSICS}

Studies of comets through the years, conducted both from ground-based observatories and Earth-orbiting spacecraft, have established the constituent parts of comets - nucleus, coma, hydrogen cloud, and tails. The cometary structure is indicated schematically in Figures $11 \mathrm{a}$ and $1 \mathrm{lb}$.

Modern theory offers a reasonably good explanation of the cometary features and their variation with time. The starting poinc is Whipple's icy conglomerate or "dirty iceball" model of the nucleus, thought to be approximately $5 \mathrm{~km}$ in diameter for Halley. Alternate ideas have been discarded by most scientists, as only the Whipple paradigm seems capable of explaining such diverse cometary features as their departures from strict Newtonian motion, their vast gas production, and their asteroidal appearance and behavior at large heliocentric distances. As a comet in its inactive state approaches the Sun in its orbit, sunlight supplies radiant energy to the nucleus, and the surface is heated. As the approach continues, the temperature of the surface layers increases to a value at which sublimation occurs. In the latter situation, most of the incident energy goes into the sublimation of ices. Usually the activity of comets begins at 2.5 to $3 \mathrm{AU}$ from the Sun. This fact together with many others indicates that the dominant constituent of most comets is water ice. Delsemme has proposed that this type of ice is probably the clathrate hydrate variety, in which minor constituents can be trapped in the cavities of a crystal lattice. The minor constituents are released when the water ice sublimates, as observed. The sublimating ice also releases the dust particles in the "dirty iceball."

The sublimated gases are mostly neutral molecules which flow away from the nucleus, dragging some dust particles with them to form the coma. Photochemical processes can affect these parent molecules anywhere. Densities near the nucleus are high enough that chemical reactions also can occur. The result is a change in the molecular species present as the gas moves away from the nucleus.

The solid particles liberated from the nucleus are of two types. The smaller particles - dust - are blown in the antisolar direction by solar radiation pressure to form the dust tail. The larger particles can orbit the Sun and scatter sunlight to produce the zodiacal light. This material is also dispersed along the comet's orbit to produce meteor showers upon entering the Earth's atmosphere. Two annual meteor showers are associated with Halley's Comet, the Orionids and the Eta Aquarids.

If sublimation of ices from a comet has been carried out for an extensive period of time, the ice supply is exhausted and the "comet" may consist of dust particles and perhaps a residual nucleus of rocky material.

The gas flowing away from the comet interacts strongly with the solar wind. For a typical comet closer than 2 AU to the Sun, an important fraction of the coma molecules are ionized and this triggers a reaction with the solar wind's magnetic field. : ssence, the ionized cometary molecules capture the interplanetary magnetic tield and cause the captured field lines to wrap around the nucleus like a folding umbrella to form the plasma tail. This process is observed because trapped ions such as $\mathrm{CO}^{+}$serve as tracers of the field 1 ines. Figure 12 .

A fairly detailed summary of the processes occurring in comets is shown in 

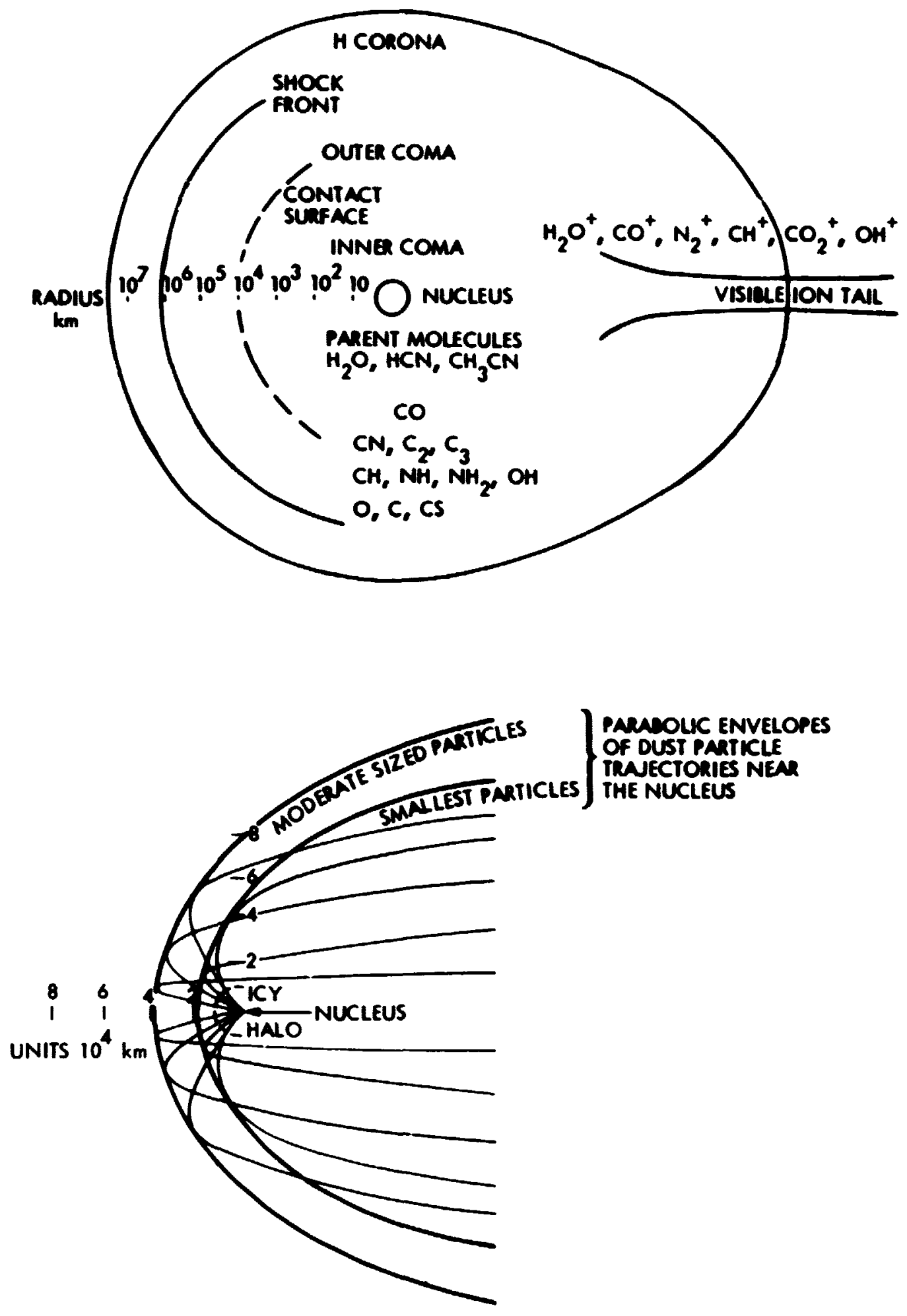

Fig. 11. Schematic drawings of the princlpal gaseous and particulate features of a typical comet on logarithmic and linear scales, respectively. 


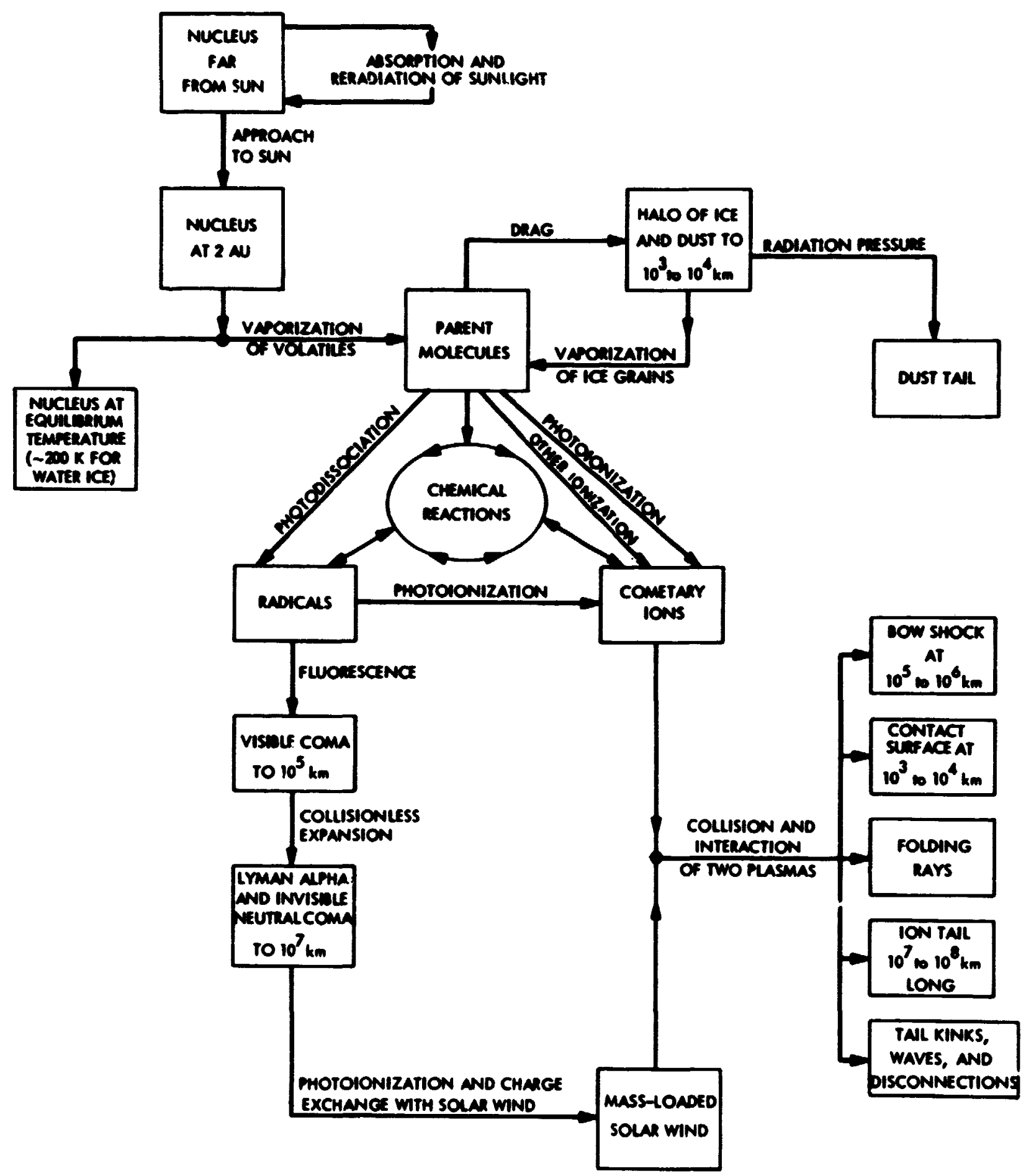

Fig. 12. Flow diagram showing features and processes involved in the interaction of a comet with sunlight and the solar wind. 


\section{SCIENTIFIC RATIONALE FOR STUDYING COMETS}

In addition to their remaikable displays and the attention that they have attracted, comets present challenging problems. Understanding their basic properties is a valuable undertaking in itself. Moreover, a deeper knowledge of physical and chemical processes occurring in these fascinating objects may well produce significant progress in other related fields.

A detailed study of a comet should provide important keys to an understanding of the origin of our solar system, and perhaps, life itself. Comets and planets probably formed from the same gas and dust reservoir. However, planets and their satellites retain at hest a blurred record of their births, obscured by billions of years of evolution during which external and internal processes have reshaped their interiors and surfaces. By contrast, comets are among the most primitive objects remaining in our solar system. Spending most of their lives on the edge of the solar system, comets have escaped many of the external processes, such as solar irradiation, that have altered the planets near the Sun. Because comets are small, they may have escaped the various internal processes that have drastically modified the structure and composition of the larger planetary bodies since their formation. In addition, the outer skin of a comet is removed during each close passage by the Sun thus exposing fresh material. Hence comets are expected to preserve the chemical and physical characteristics with which they (and the rest of our solar system) were formed.

Conets may have been a major source of organic materials in the atmospheres of the terrestrial planets. Thus a study of their composition could provide clues to the nature of the prebiological environment of Earth. Some cometary molecules may be the precursors of terrestrial life - although this is only speculation and requires additional data.

Sirce the composition of comets appears to be similar to that of interstellar clouds, the study of comets may help solve major problems concerning molecule formation and the nature of interstellar dust, clouds, and dark nebulae.

Observations of comets have already led directly to the discovery and subsequent study of the solar wind. Studies of comet/solar wind interactions could provide deeper understanding and improved physical models that would allow each returning comet to be a "free" probe of the interplanetary medium through which it travels, as well as a probe of basic processes in plasma astrophysics. 


\section{WHY STUDY COMET HALLEY?}

With a period of approximately 76 years, Comet Halley has apparently made elatively few returns to the solar neighborhood since being perturbed in from the cloud of conets surrounding our solar system. It is one of the "freshest" comets with a period less than 200 years, and it is the only short-period comet known to display the full range of cometary phenomena (i.e., large, dense coma, both ion and dust tails, jets, and streamers). Other short-period comets do not show such a range of activity and appear to be much nearer the end of their physical evolution. Halley is the only really active comet that also has a we11determined orbit and has demonstrated fairlv reltable behavior over a period of at least two millennia. In short, Comet Halley is the only fully active comet that can be fredicted with confidence to return in our lifetime. Other shorter period comets such as Encke, Tempel 2, d'Arrest, Giacobini-Zinner, and TuttleGiacobini-kresak are excellent targets for detailed geochemical and morphological studies of evolved nuclet and inner come, but they cannot be expected to have highly active nuclei or well-developed onospheres and tails.

Substantial data concerning Halley was gathered at the 1909-1911 apparition, primarily direct photographs and spectra. The classic summary of the comet's properties at the 1910 apparition was given by Bobrovnikoff in 1931.1 Other conpilations are currently in preparation. A well-planned study of Comet Halley at the upcoming apparition will provide the unique opportunity to compare the properties of a bright and active comet at two successive appearances. 1910-1911 observations of Comet halley are shown in Figs. 13 through 19. 


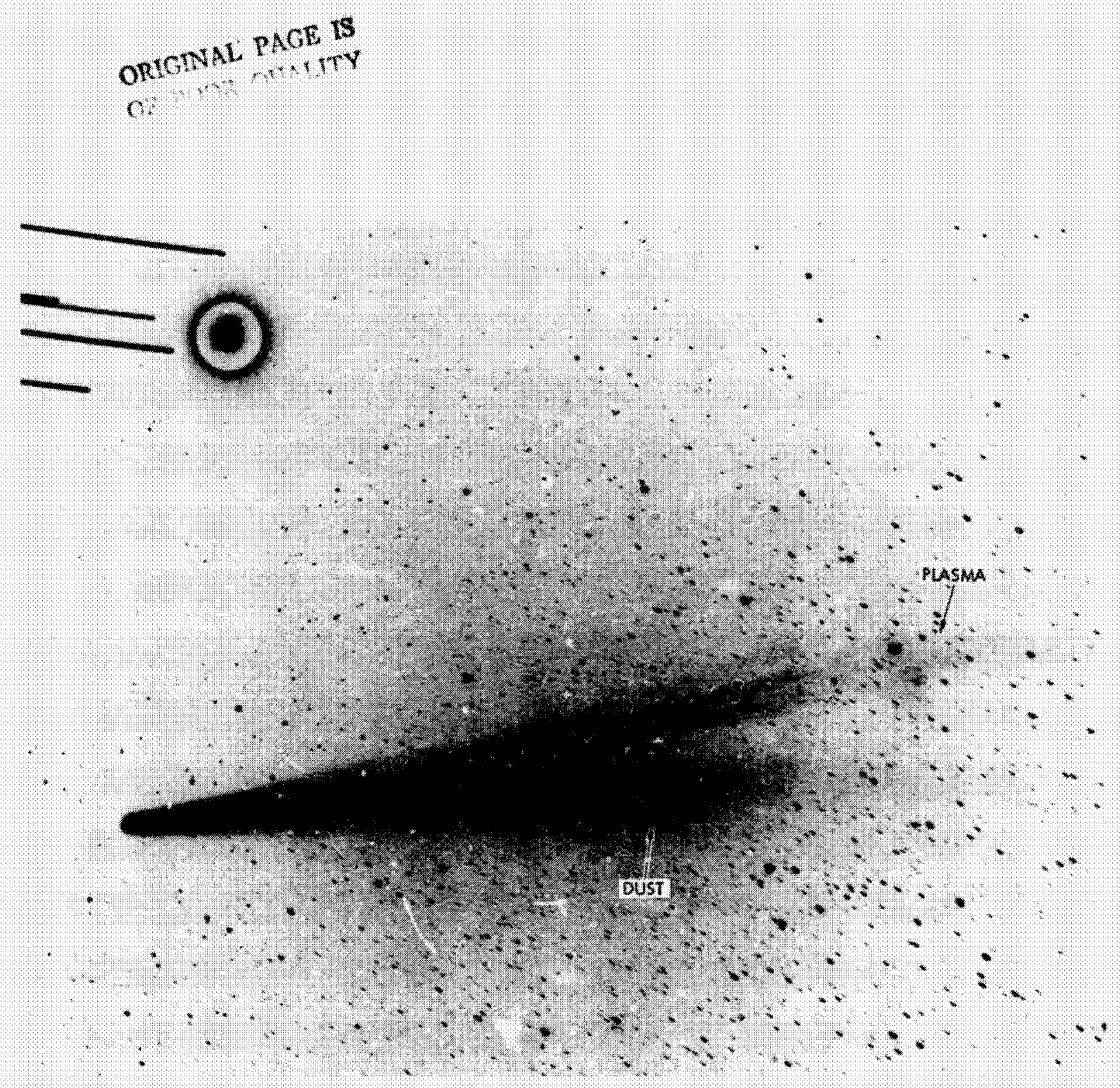

Fig* 13. The two tails of a comet - the fuzzy dust tall and the stralght plas 1 tail -- can be seen in this photograph of Comet Halley, which was obtalned on Nay 6, 1910, at the Lowell observatory in Flagstaff, Ar Lzona. The comet was $0,7 \mathrm{NU}$ from the Sun and $0.6 \mathrm{AL}$ from the Earth (Lowell obstervatory photograph). 


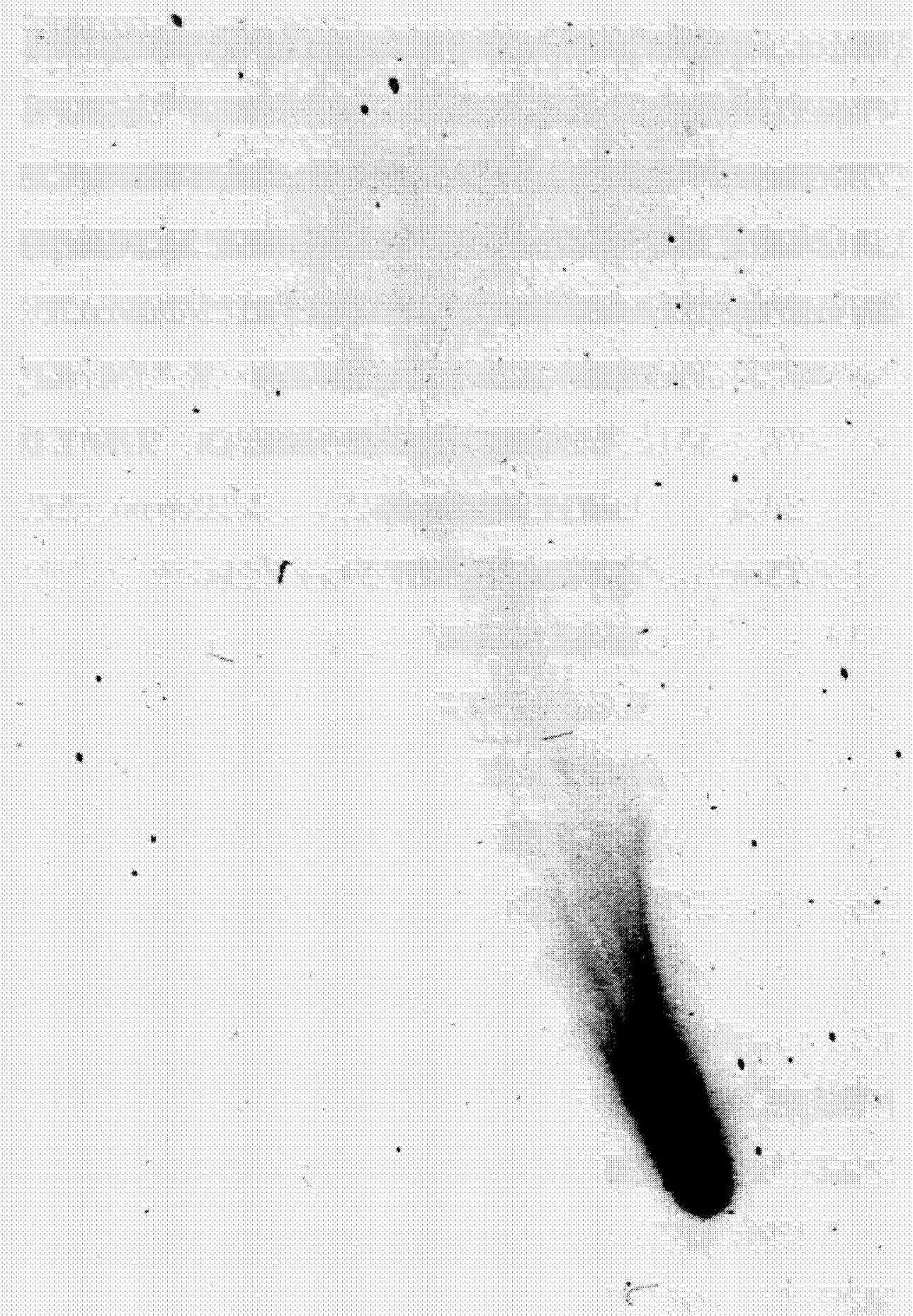

Fig. 14. A pronounced disturbance in the ion tall of Comet Halley appears on this photograph, obtained on April 22, 1910, at the Kodaikanal observatory in India. The neliocentric and gevcentric distances amounted to 0.6 and $1.1 \mathrm{AU}$, respect ively. 
ORIGWAL, PACr is

of Poon GuALTYY

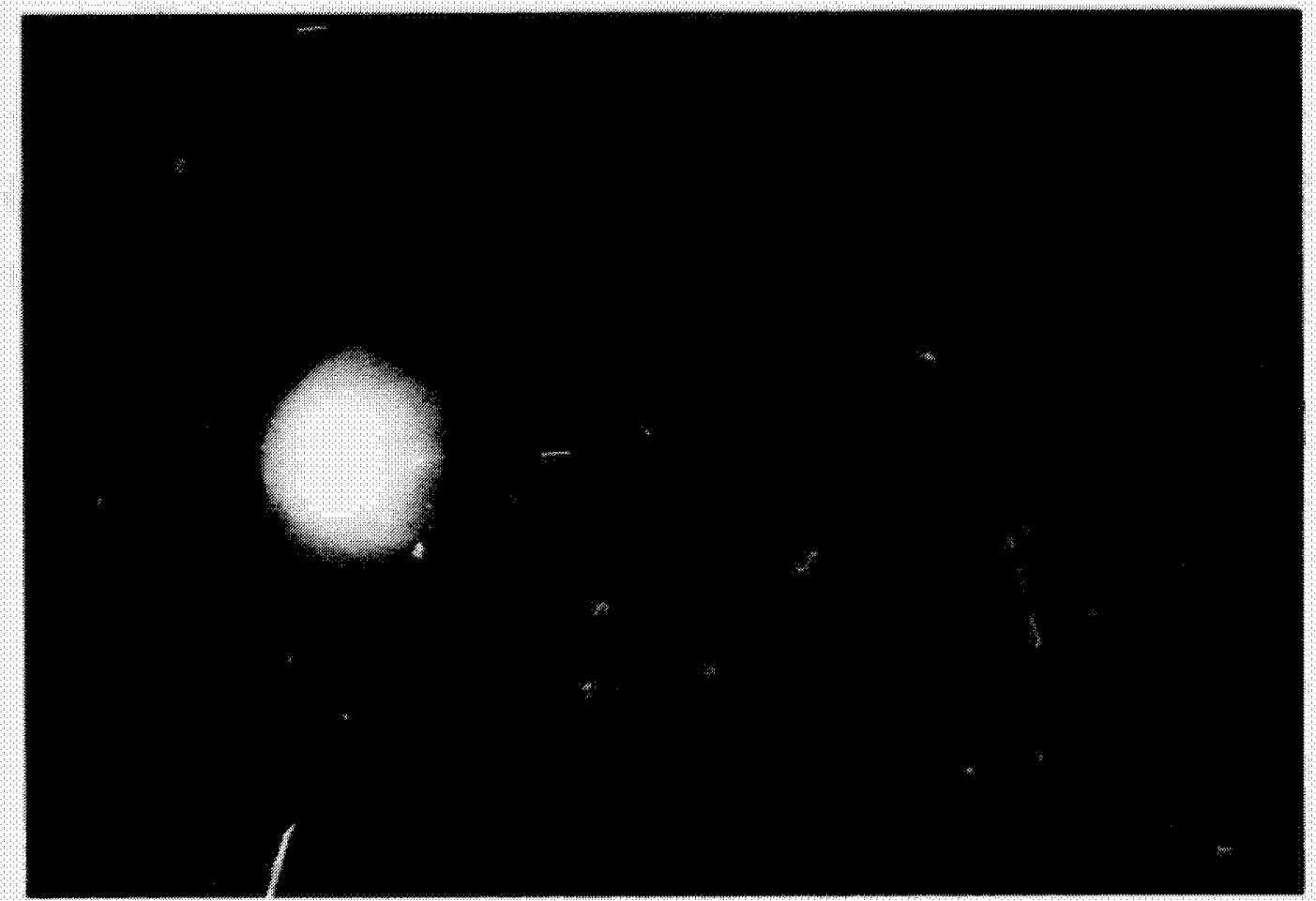

718. 15a. Detal'ed structures and fountainlike jets can be recognized in the head of Comet Halley on this photograph. It was obtained on Yay 25, 1910, at Helwan Observatory in Egype. when the conet was $0.9 \mathrm{AH}$ I roa the Sun and 0.2 At fron the Earth. 


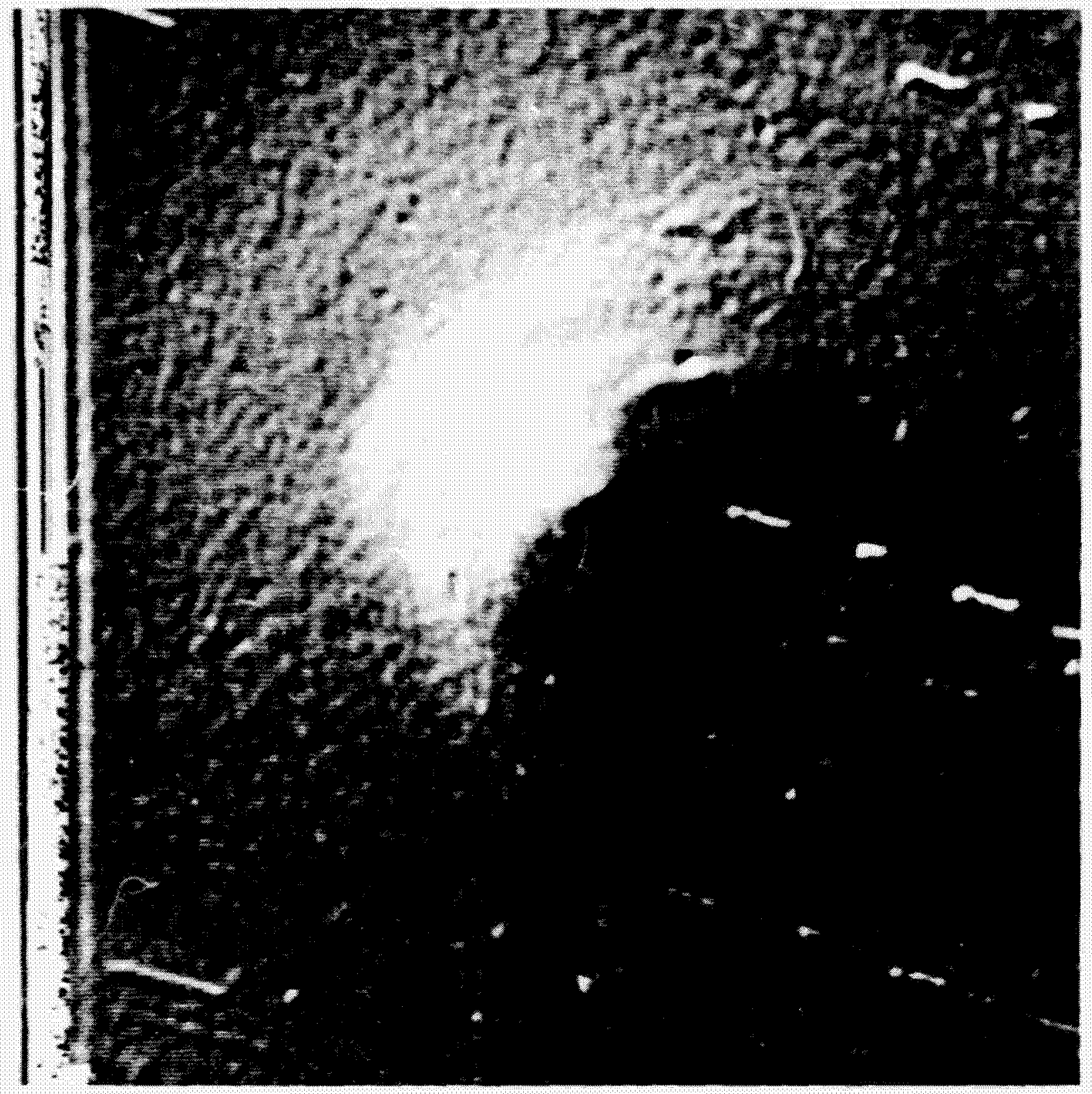

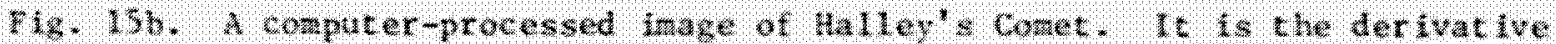

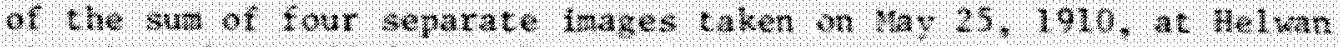

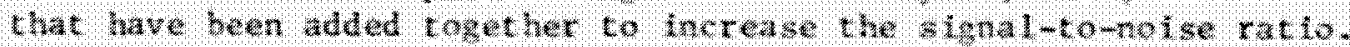

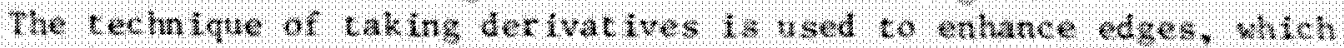
In this instwee are prowinent in the envelopes on the stumard side of the nut letrs. 

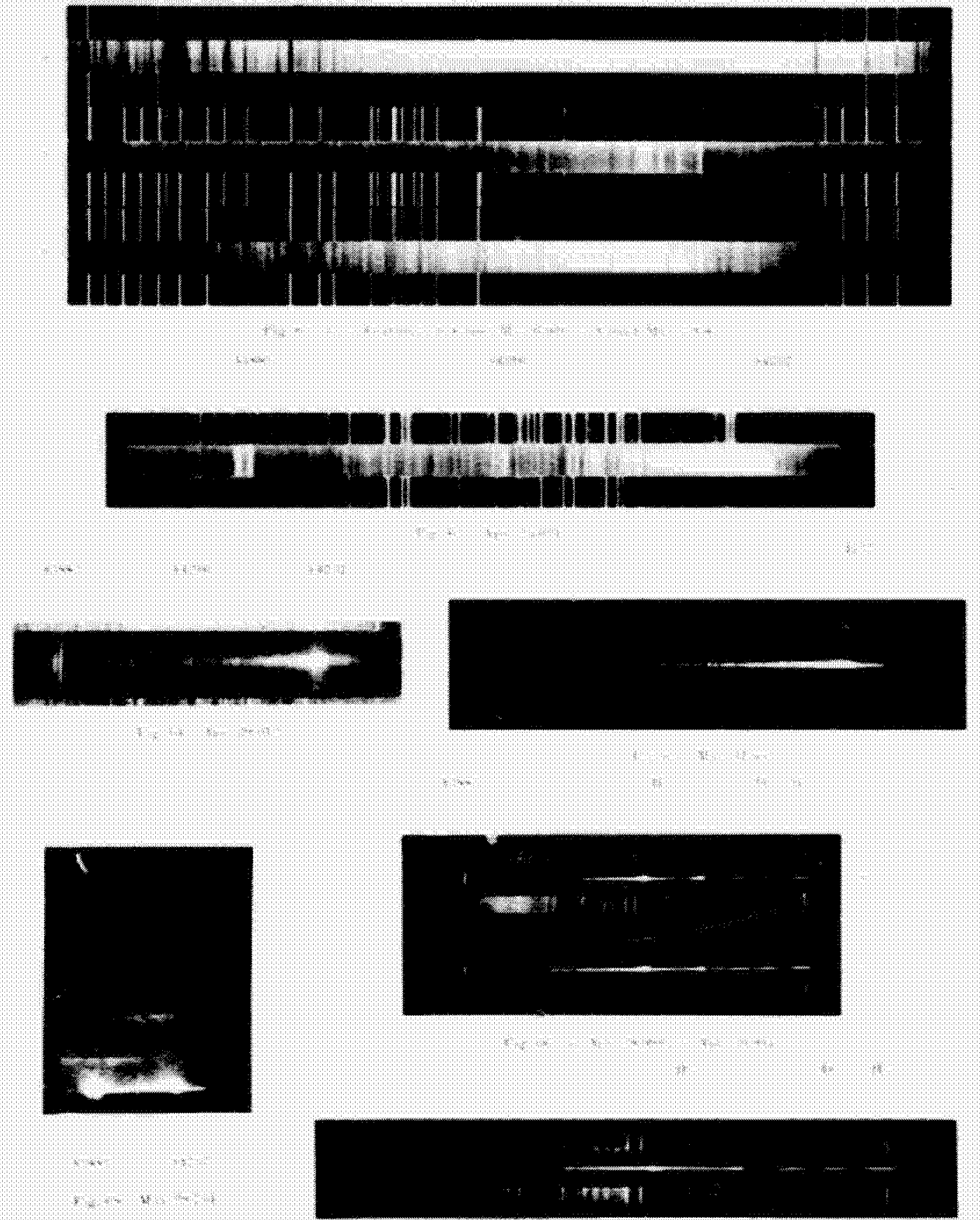

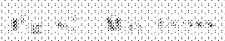

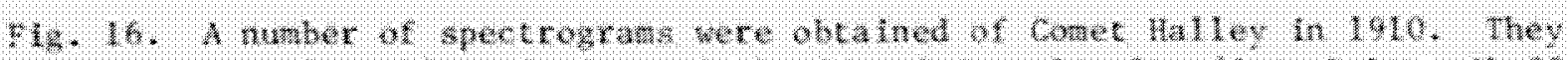

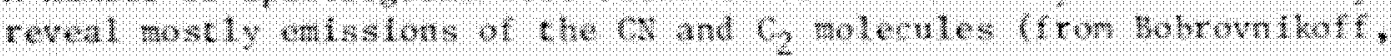
1411 . 


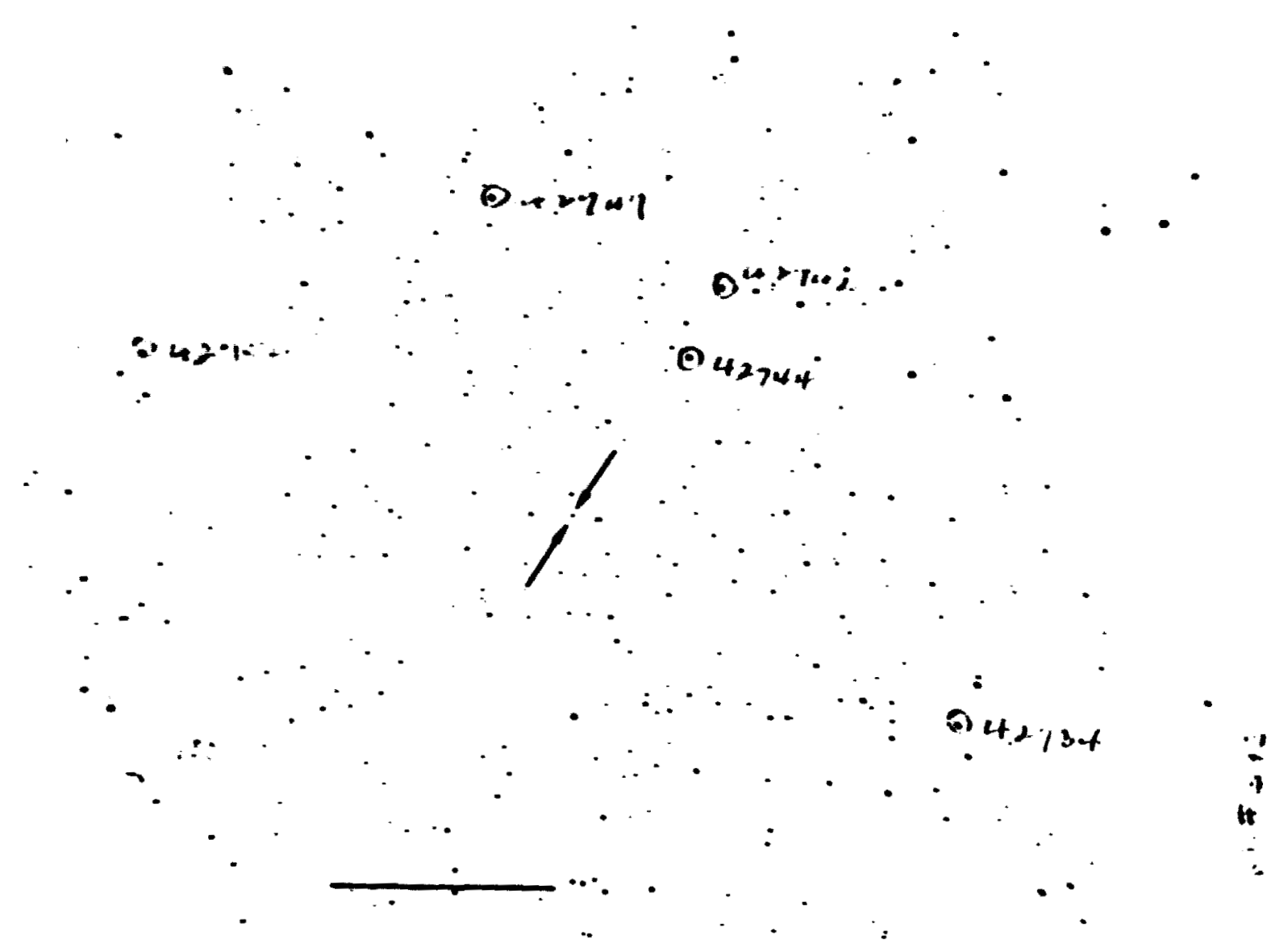

Fis. 17. Comet Halley on its way back into the deep-freeze of interstellar space. The figure shows one of the last photographs of this comet obtained at Lowell Observatory in Flagstaff, Arizona, on May 30, 1911, when the comet was at about Jupiter's distance from the Sun. The position of the comet is marked. 
ontcin: $\cdots \cdots:$ is

OE POulk Quntiry
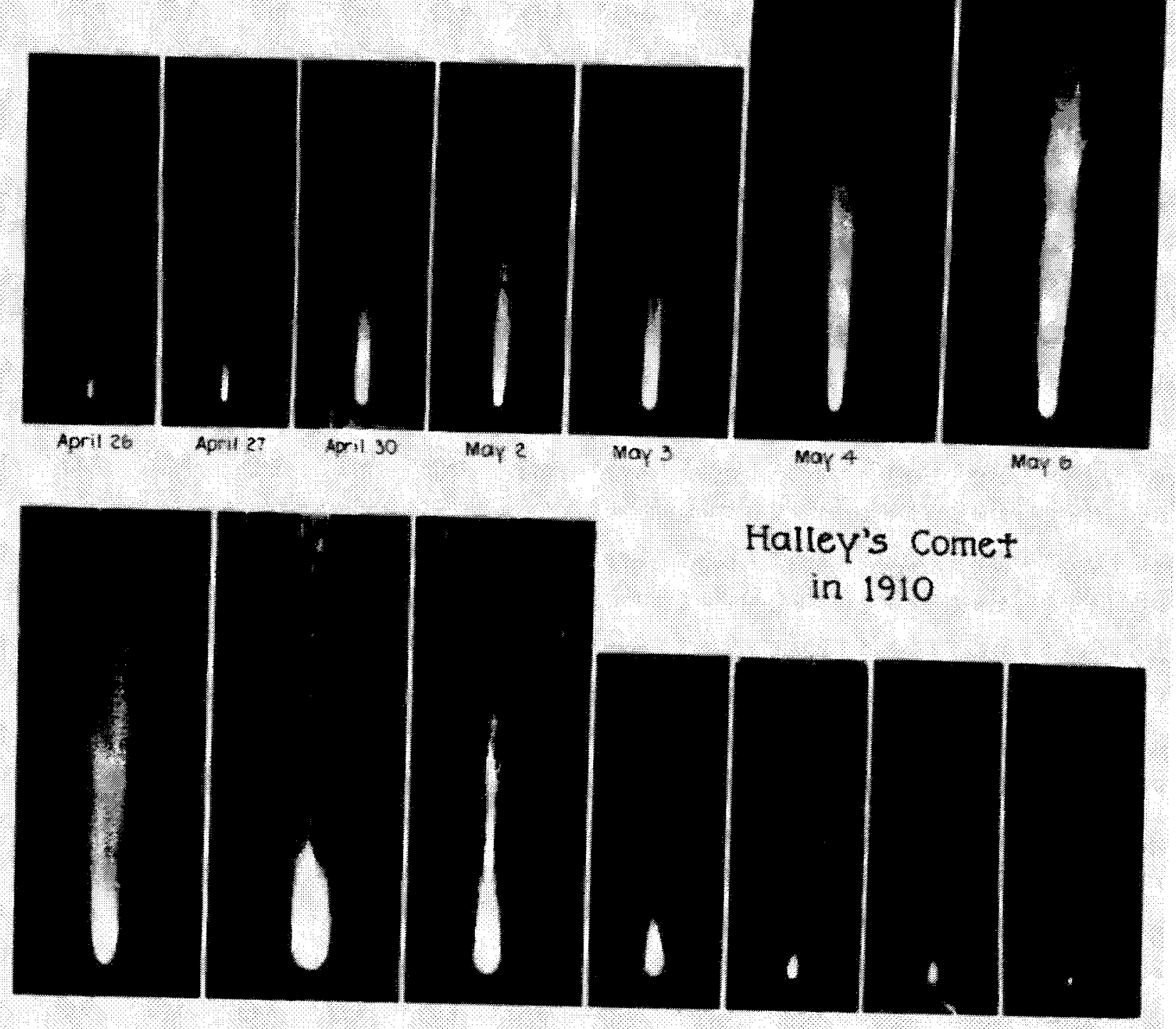

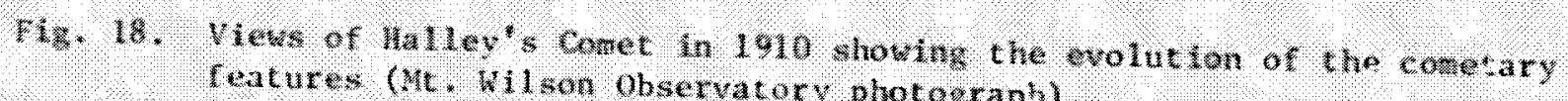
features (Mt, Whlson observatory photogerapl).

3 


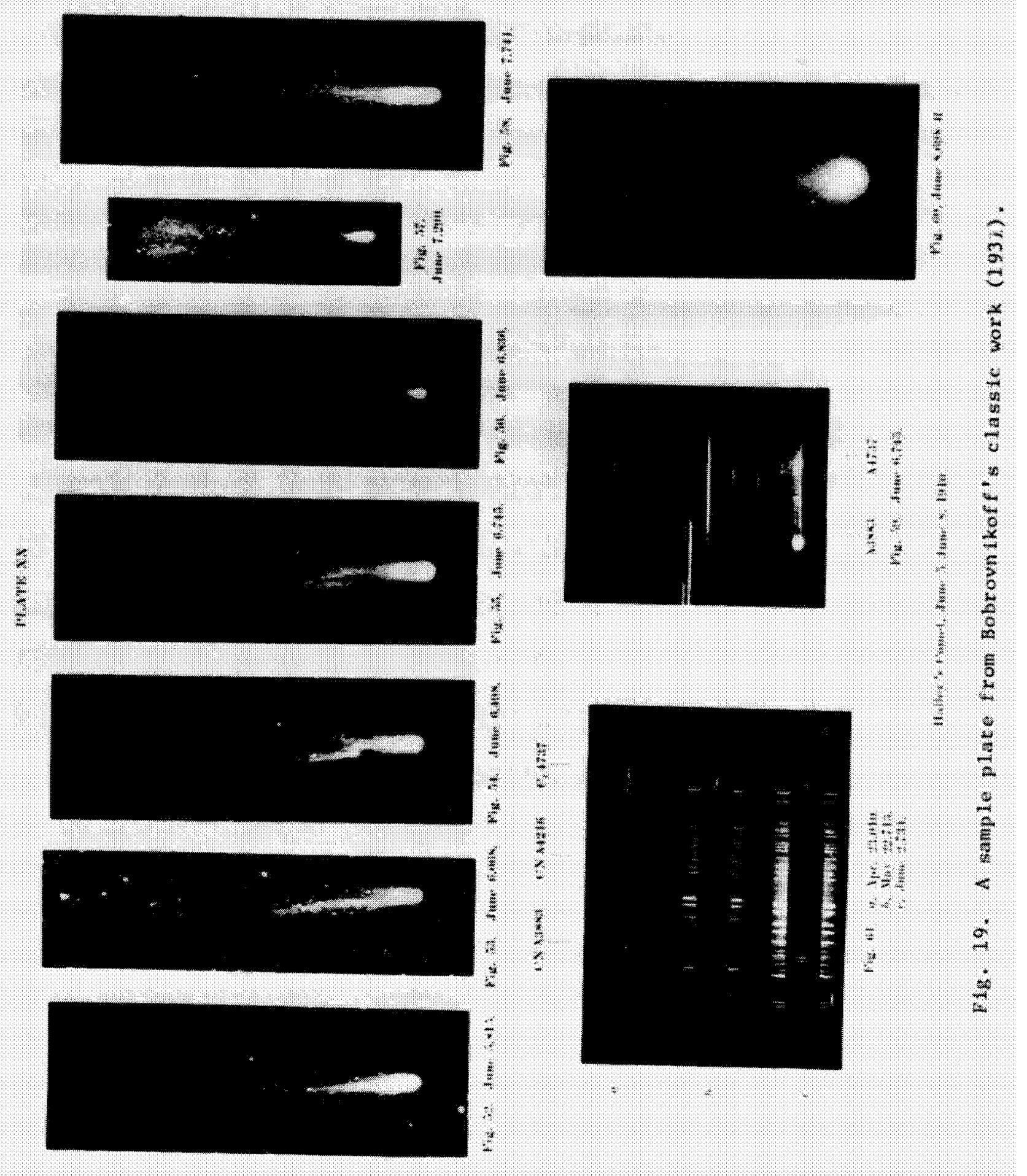




\title{
VI. SCIENTIFIC OBJECTIVES OF COMETARY RESEARCH IN THE 1980s
}

\begin{abstract}
Objectives for the upcoming decade are straightforward and constitute a consensus in the scientific community. They have been enunciaced by numerous study groups including the Comet Halley Science Working Group - 1977, the Comet Science Working Group - 1978, and the Comet Science Working Group - 1979.
\end{abstract}

The objectives are:

(1) To characterize the structure, basic physical processes, and chemical nature of cometary nuclei, and to determine the changes that occur as a function of time and orbital position.

(2) To characterize the basic properties of the cometary atmosphere and ionosphere and their development with time and orbital position, including production, structure, extent and changing chemical composition.

(3) To determine the nature of comet tails and the processes by which they are formed, and to characterize the interaction of comets with the solar wind.

Many of these objectives can only be met by a flight program, but study of time variations then requires a rendezvous space mission, which is no longer an option for Comet Halley. The best option remaining is the use of a flyby mission to calibrate synoptic observations made from earth-based and earth orbital observatories. 


\section{INTERNATIONAL HALLEY WATCH PURPOSE AND ORGANIZATION}

The purpose of the International Halley Watch is to promote worldwide cooperation, communication, and standardization (where useful) in studies of Halley's Comet. Coordination and communication extend from the present planning and preparation phase through the observation period to include archival publication of all scientific Halley observations. The goals of the IHW are:

(1) To stimulate, encourage, and coordinate scientific observations of Comet Halley throughout its entire apparition.

(2) To insure that the observing techniques and instrumentation are standardized whenever appropriate.

(3) To insure that the data and results are properly documented and archived.

(4) To receive and distribute data to participating scientists and provide information to the public and media.

(5) To stimulate relevant instrument development where necessary. The IHW is intended to be international in scope and, in addition to supporting observations of the comet by ground-based observers, the IHW will actively encourage observations made from deep space and Earth orbit.

The organization proposed in Fig. 20 is offered as the simplest one adequate for these goals and for the accomplishment of the scientific objectives. Since the IHW is dedicated to maximizing the scientific return from the study of Halley it will be important that it coordinate closely with space project activities even though the IHW itself concentrates especially on traditional groundbased observation. This is reflected in the proposed organizational structure.

The IHW enables an important symbiosis between ground and space observations. Observations made during encounter missions are vital for deriving maximum scientific benefit during the Halley apparition, and the development of good dejicated missions and the use of spacelab opportunities are strongly recommended.

Each major ground-based discipline will be represented by a specialist who, in consultation with other experts in his field, will recommend specific objectives, standards, and priorities for observations made in that discipline. These discipline specialists will be the organizational counterparts of the project scientists for each major flight activity such as Spacelab, Space Telescope, and each space probe to Halley. Also on this organizational level between observers and the lead of fice will be individuals who act as IHW contacts with amateurs and with planetaria.

The office of the Halley Watch Leader will be responsible for communication among the various groups, for setting data formats, for the preparation of the ultimate archival publication of all results obtained, for preparation of information about Comet Halley appropriate for distribution at various levels of 


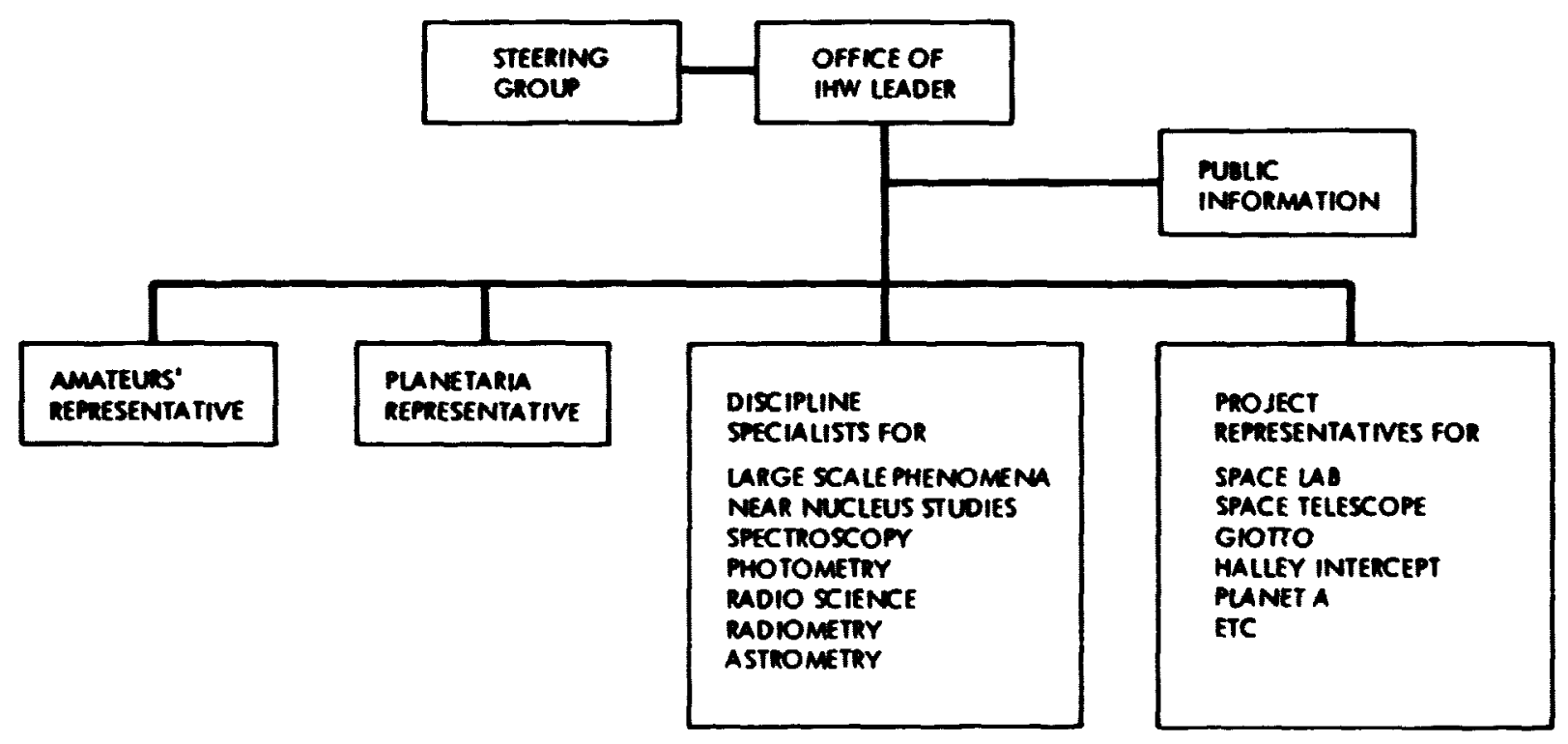

Fig. 20. International Halley Watch organization outline. 
scientific sophistication (professional, amateur, general public), and for solving unexpected problems that may require additional attention. In cooperation with discipline specialists, the Lead Office will establish Halley Watch Days when special emphasis will be given to obtaining simultaneous observations in all disciplines. Halley Watch Days would be particularly important before, during, and after spacecraft flybys of Comet Halley. In general, the office of the Halley Watch Leader will do everything possible to assure a successful halley Watch program.

The bywords of the IHW must be stimulation, cooperation, encouragement, .nd understanding. Although each discipline specialist ultimately will recommend certain standards and priorities and will create a net of observers who agree to follow those recommendations at least in part, net members will be encouraged to undertake any other work on Comet Halley that may seem reasonable to them. Some observers will make important and unique observations of the comet that do not fall within any of the ground based nets of the IHW. These observers and those astronomers who cannot or choose not to join a net will still be helped in any way possible and encouraged to contribute their data to the final Halley archive, which will be available in printed form to all scientists. A major task of the discipline specialists will be to stimulate and coordinate such an exciting program that most scientists will wish to participate. The greatest assets of the discipline scientists will be scientific rationality, flexibility, diplomacy, and enchusiasm.

A steering group should be set up to advise the Halley Watch Leader and to assure proper communication with appropriate scientific and political organizations. Among Steering Group members would be representatives of the IAU and COSPAR. NASA and the NSF would be represented as major funding sources. If governments of other countries decide to financially support participation of their astronomers in this activity, it may be appropriate to have them represented as well. On the other hand, every country with astronomers participating in a net cannot have a steering group nember or the group would become unmanageably large. The Halley Watch is intended solely as a cooperative effort among scientists to maximize knowledge of Halley's Comet. The participation of political entities on the Steering Group would be only for the purpose of aiding the scientific goals by communicating scientific needs to appropriate funding agencies.

A public information office will be located at the institution of the Halley Watch Leader. Its principal purpose will be to prepare material for distribution to the general public and media on the goals and progress of the Halley Watch and on the results obtained or expected from the comet observations. It also will work with the planetaria representative to prepare material suitable for planetarium use. The Public Information office will work with the Halley Watch Leader to prepare a regular newsletter for all net members, and it will supply any net member with material suitable for public lectures. In general, it will attempt to fulfill the nonprofessional communication needs of the Halley Watch at all levels. 


\section{INTERACTION OF THE IHW WITH OBSERVING GROUPS}

As indicated in Table 1 the IHW will be interacting with various groups of observers - deep space mission experimenters, investigators using near-Earth observations, and ground-based observers - both professional and amateur.

\section{A. DEEP SPACE OBSERVATIONS}

Several space agencies are studying possible flyby or fiythrough missions to Comet Halley in 1985-86. Until recently, U.S. plans nave centered around a solar electric propulsion stage carrying a rendezvous spacecraft designed for use primarily during a rendezvous with short periodic comet Tempel 2 in 1988 . This rendezvous spacecraft was to deploy a European Space Agency probe during an enroute flyby of Comet Halley in late 1985. However, present NASA planning does not include funding for the necessary solar electric propulsion stage. Accordingly, planning has begun for a ballistic mission to Comet Halley. A later comet rentezvous would be provided when the solar electric propulsion stage is readied. While many trajectories and mission plans are possible for a Halley flyby mission, the U.S, is currently considering a flyby with a 3-axis-stabilized spacecraft with Voyager class imaging and eight nonimaging instruments as the primary scientific instrumentation. The European Space Agency (ESA) has approved a rid-March 1986 flyby mission to Halley using a spinning Geodetic Earth-Orbiting Satel1ite to be launched by an Ariane rocket. Primary planning for this ESA mission (called "Giotto") calls for eight science instruments on the spacecraft.

The Japanese are planning to launch their first deep space probe (called Planet A) to take large-scale observations of comet Halley with at least tro instruments. The Soviets and French are studying a mission which would use the balloon delivery spacecraft of the 1984 Venera mission to continue on to flyby Halley in late February or March 1986. The flyby spacecraft will be 3-axisstabilized. The potential science instrumentation is not known by us at the time of this writing.

Although the IHW must proceed whether or not there are deep space Halley missions, it is recognized that such missions can provide the greatest science return during the coming apparition. No near-Earth instrument will ever see the nucleus of Halley as anything but a point of light, leaving to hypothesis all questions of nucleus structure and morphology. Only those inner coma components having a strong spectral signature can be detected from Earth, and then with some spatial ambiguity, leaving to hypothesis the nature of most parent molecules and much coma chemistry. The magnetohydrodynamics of a comet's interaction with the solar wind would remain a subject for speculation without an encounter. Once a spacecraft quantitatively measures the physical conditions and mechanisms occurring in Halley at even a single point in time, there is hope for far better understanding of all of the other observations taken throughout the apparition.

\section{B. EARTH ORBITAL OBSERVATIONS}

Two of the most promising platforms for observing Comet Halley are the Space Telescope and Spacelab. The IHW should actively encourage individual 


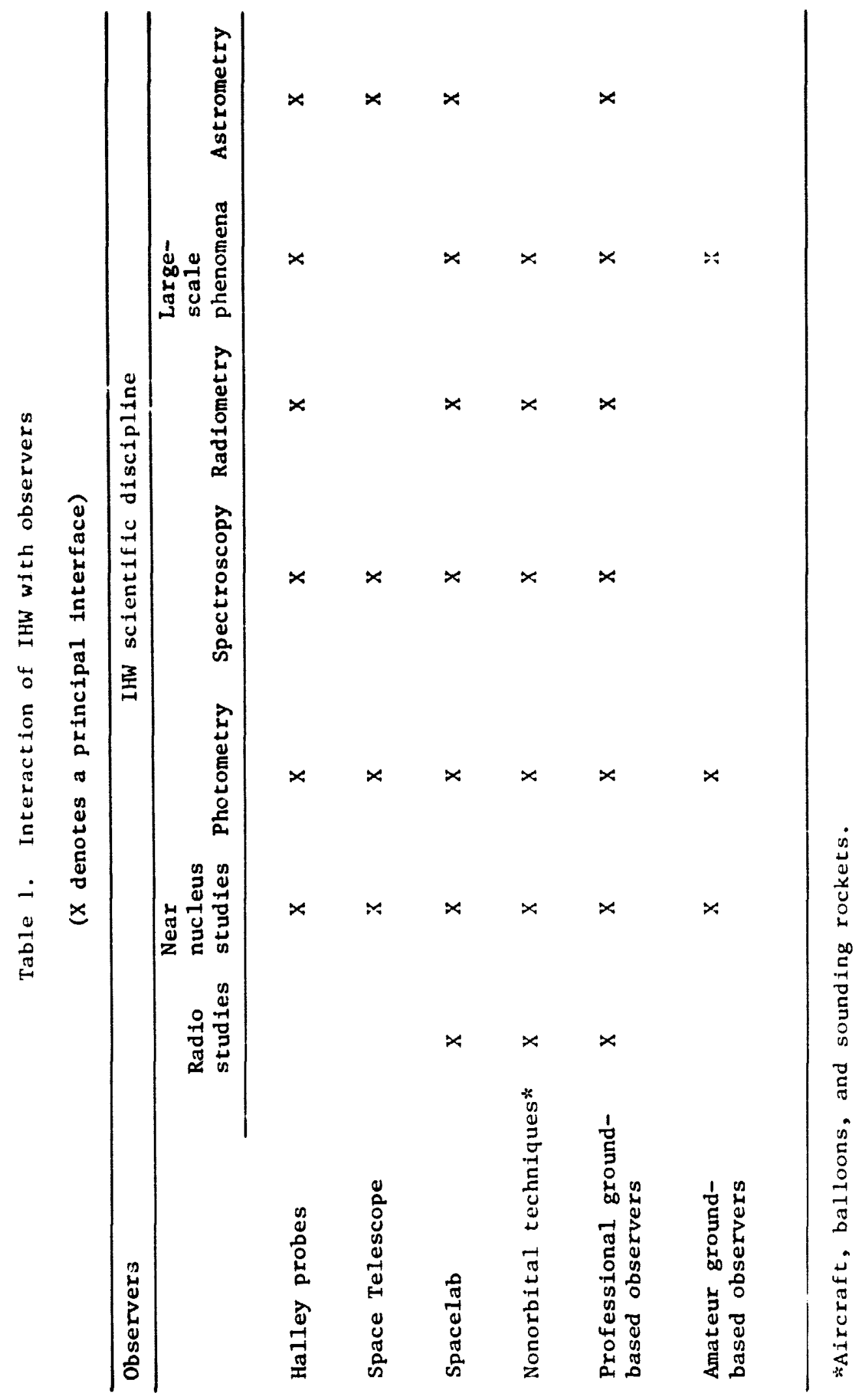


experimenters to observe comet Halley for as long a time period at as many different wavelengths as possible.

The Space Telescope is scheduled for launch in late 1983 or early 1934 and It probably will be the only free-flying spacecraft capable of making conet observations throughout the Halley apparition. The Space Telescope will not be able to point within $50^{\circ}$ of the Sun and its field of view is 1 imited to 2.7 arc minutes. Phenomena such as the evolution of a radical species as a function of radial distance from the comet would have to be studied with several consecutive narrow field observations. Nevertheless, the Space Telescope should make extrenely valuable observations of the comet at large heliocentric distances and into about $0.9 \mathrm{AU}$ when the comet gets within $50^{\circ}$ of the Sun. The wavelength sensitivity of Space Telescope instrumentation from about $1200 \AA$ to $1.1 \mu$ should permit spectroscopic studies of various small-scale phenomena over Iong periods of time both before and after perihelion. The unprecedented sensitivity of the 2.4-meter Space Telescope will allow monitoring of the comet's complete activity cycle. This cycle begins with the comet evolving from a quiescent frozen nucleus to an active comet throwing off enormous quantities of gas and dust; it ends after the impressive cometary phenomena has ceased and the comet has returned to the frigid region whence it came.

Spacelab instrumentation could effectively be used to study cometary evolution during the crucial period around perihelion passage - a period when the comet is unobservable from the Space Telescope. This gap in coverage could be filled by specialized instrumentation designed to observe the comet within a few degrees of the Sun. Shuttle flights dedicated to observing Comet Halloy would be prohibitively expensive. However, the shuttle offers the opportunity of flying modified off-the-shelf instrumentation on speciul pallets aboard scheduled Shuttle flights, sharing expenses with other payloads. - we hardware already approved for Spacelab may be suitable for a Comet Halley package with little or no modification. Some new instrumentation may have to be developed or some existing instruments that have not yet been selected for Spacelab could be added to the Halley pallet. It appears practiral to include such opportunities in the Spacelab schedule. The IHW would act in an advocacy role with NASA Headquarters to ensure that a smet instrumentation package is integrated and scheduled on the Shuttle manifest. An example spacelab pallet payload for comet observations is presented in Table 2 .

In addition, although not an of $f-t h e-s h e l f$ instrument, a millimeter wave spectrometer operating from the Spacelab would offer an excellent way of studying the production of water as a function of time and heliocentric distance. The strong rotational transition of water at $183 \mathrm{GHz}$ is ideal for this purpose. of crucial importance to a conet's evolving energy balance, the water production rate varies with heliocentric distance and responds to brief outburst events. Hence, extended duration observations would be required.

Ir the infrared wavelength region, the Infrared Astronomy Sate11ite (IRAS) and the Shuttle Infrared Telescope Facility (SIRTF) may provide valuable observations. IRAS is scheduled for one year of activity beginning August 1981. However the launch date may slip by six months or more, and the one-year predicted lifetime may actually be extended. Under this combination of circumstances, IRAS 
Table 2. Spacelab - Sample payload for comet observations

\begin{tabular}{|c|c|c|c|c|c|}
\hline Experiment & $\begin{array}{l}\text { Field of } \\
\text { view (deg) }\end{array}$ & $\begin{array}{l}\text { Angular } \\
\text { (spatial) } \\
\text { resolution }\end{array}$ & $\begin{array}{l}\text { Spectral } \\
\text { resolution } \\
(\lambda / \Lambda \lambda)\end{array}$ & $\begin{array}{l}\text { Spectral } \\
\text { range }\end{array}$ & Notes \\
\hline $\begin{array}{l}\text { Wide-field } \\
\text { imag+ng }\end{array}$ & 5 & $\begin{array}{l}22.5 \text { arc-sec } \\
\left(1.6 \times 10^{4} \mathrm{~km}\right. \\
\text { at } 1 \mathrm{AU})\end{array}$ & 10 & $0.115 \mu \mathrm{m}-1.1 \mu \mathrm{m}$ & 1 \\
\hline $\begin{array}{l}\text { High-resolution } \\
\text { imaging }\end{array}$ & 0.5 & $\begin{array}{l}2.25 \text { arc-sec } \\
\left(1.6 \times 10^{3} \mathrm{~km}\right. \\
\text { at } 1 \mathrm{AU})\end{array}$ & 10 & $0.115 \mu \mathrm{m}-1.1 \mu \mathrm{m}$ & 2 \\
\hline $\begin{array}{l}\text { High resolution } \\
\text { spectroscopy }\end{array}$ & 0.5 & $\begin{array}{l}\text { approx. } 10^{3}- \\
10^{4} \mathrm{~km} \text { at } 1 \mathrm{AU}\end{array}$ & $10^{4}$ & $0.115 \mu \mathrm{m}-$ "vistble" & 3 \\
\hline
\end{tabular}

Notes:

1. Baseline detector is assumed to be an $800^{2}$ pixel, coronene-doped CCD. The nearest existing instrument to this specification is the (aallleo CCD camera (FOV $=3.2^{\circ}$ ). Note that the baseline Galileo camera design would reyuire extensive modification to gain UV throughout, 1.e., substitution of UV-transmitting or all reflective optical elements for glass elements in baseline design.

2. The Spacelab UV Imaging Telescope (T. Stecher, Principal. Investigator) should approximately match this angular (spatial) resolution and FOV specificution. However, the baseline design for that instrument uses CsI and CsTe image tubes for h1gh sensitivity in the 0.12-0.28-pm spectral range, with no sensitivity longward of $0.28 \mathrm{\mu m}$.

3. The Spacelab-1 Imaging Spectronetric Observatory (1NS001) covers the spectral range 0.02 to $1.20 \mu \mathrm{m}$ at resolution $\lambda / A \lambda \leqslant 10^{3}$. However, this Instrument's pointing capabilities are limited by the fact that it is hard-mounted to the pallet, and therefore depends on the spacecraft Acs. The Spacelab UV Spectroscopy Experinent (A. Davidson, Principal Investigator) would have better pointing capability, $\lambda / \Lambda \lambda \simeq 10^{2}$ and spectral range 0.11 to $0.19 \mu \mathrm{m}$, with possible extension to $0.3 \mu \mathrm{m}$. 
still could be operating in late 1983 and would be capable of observing Halley then at about 8.5 AU from the Sun. The SIRTF would be an extremely sensitive instrument but its usefulncss would be limited by its late date of first operation, in June 1986 .

The project scientists of all relevant and cooperating projects (f lyby missions, Planet A, Space Telescope, Spacelab, IRAS, SIRTf, etc.) will act as the of icial liaisons between their projects and the IHH.

\section{NONORBITAL FLIGHT TEChNIQUES}

Good use has been made in space research of a number of nonorbital flight techniques involving aircraft, balloons, and sounding rockets, over the years, NASA has used a number of aircraft as observing platforas. the nost advanced of these being the C-141 cype cargo jet outfitted as the Kuiper Airborne Observatery. This has been particularly important in carrying infrared and eicrowave detectors above the troposphere with its high content of water vapor wich absorbs strongly throughout wuch of the spectral region from $0.8 \mathrm{\mu t}$ to $1.35 \mathrm{~cm}$.

In a completely different cechnique. Brownlee has utilized the tigh-altitude U-2 aircraft for the direct collection of wicroweteoroids for geochemical analysis. While very successful, the technique of fers no sure way to separate conetary particles from sporadic incerplanetary material. The ass flux increase during large annual meteor showers is on the order of $10 \%$ or less. Some of this mass will survive as micron-sized welt droplets wich can be collected in tise stratosphere using the existing, routine aircraft collection cechniques. Identification of a Halley component could be atteapted by detectins changes in the population of collected particles. In particular, because of the high entry velocities, all of the shower particles should have been heated to high levels during entry, and most size ranges would have been melted to form spheres. Even though shovers may not originally contain small particles, swall debris will be formed by fragmentation of the larger bodies. A possible example of a particle from Comet Halley is shew in Fig. 21 .

Balloons have heen used in several countries to raise payloads to altitudes near $40 \mathrm{~km}$ in order to overcome atmospheric absorption and turbulence for periods of hours. This is about $15 \mathrm{~km}$ above the altitude possible with aircraft using air-breathing engines, but the balloons pay a penalty in decreased flexibility and reliability and increased cost per flight.

Sounding rockets have been in common use in many countries to carry small payloads to altitudes above $100 \mathrm{~km}$ for periods of five minutes or more. There is no ozone abscrption above $100 \mathrm{~km}$, making ultraviolet studies from $0.1-0.3$ um quite practical during the 1 imited time the rockec is high enough. In 1972 a rockerborne mass spectrometer measured metallic ions in the mesosphere which were attributed to comet Encke (Beta Taurids). Continued experimentation possibly could determine elemental abundance data for the Halley showers.

All of these flight techniques could be used to take useful data on comet Halley. Their actual use will depend in some cases upon the instruments available in orbit and in many cases upon cost-benefit studies not yet carried out. 


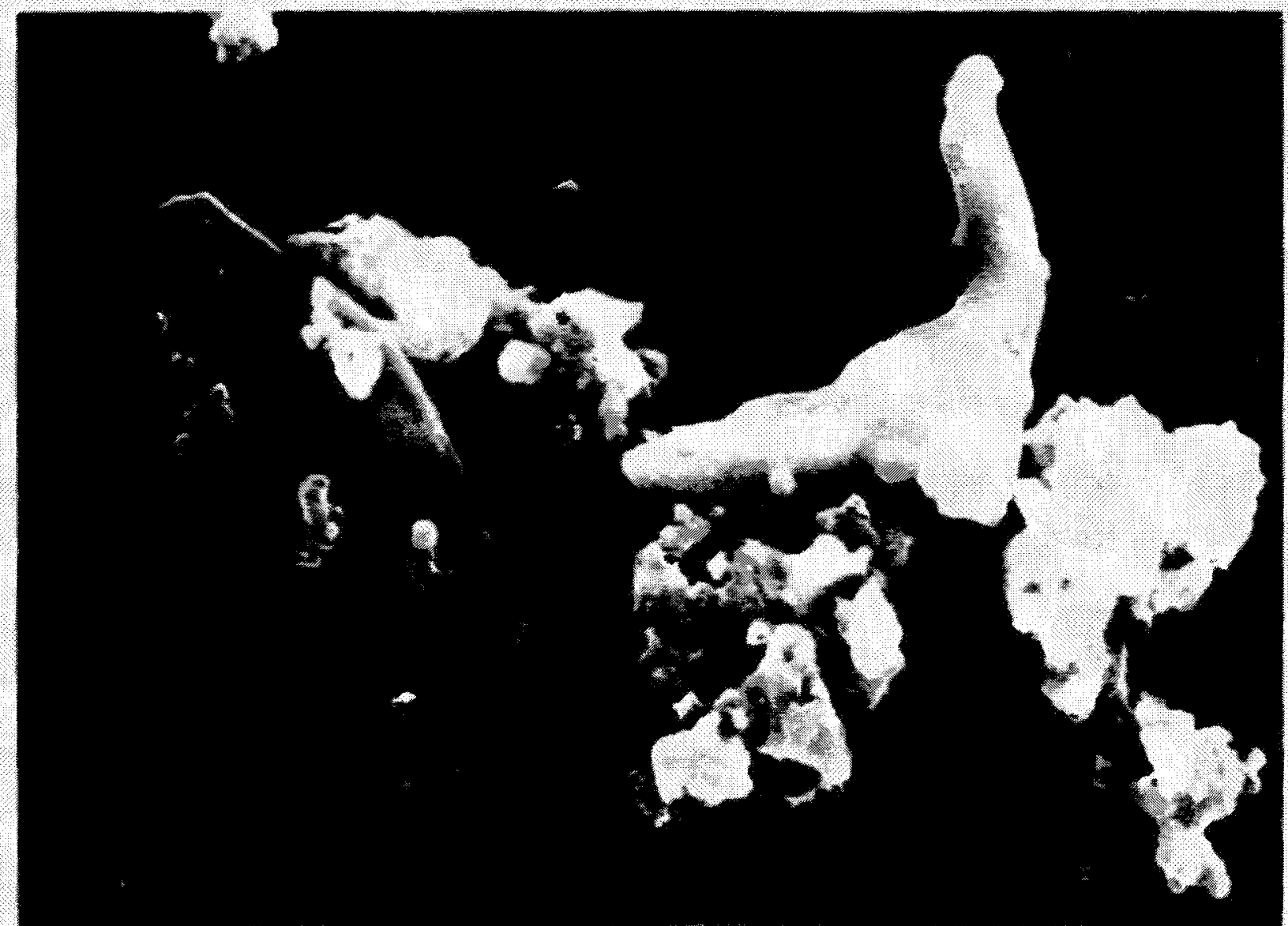

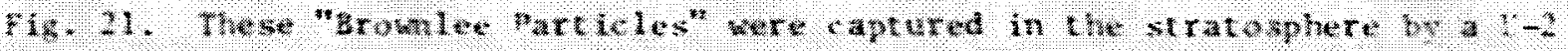

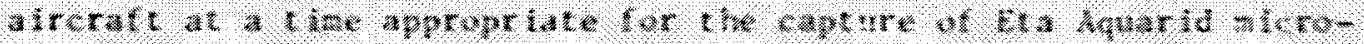

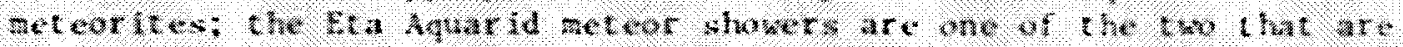

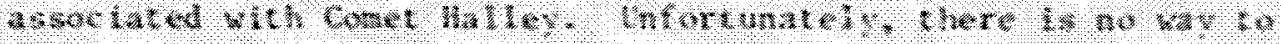

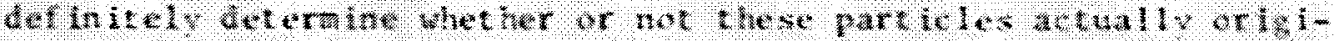

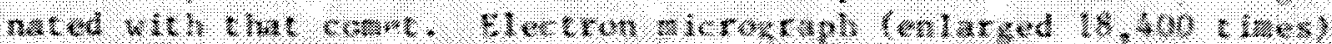

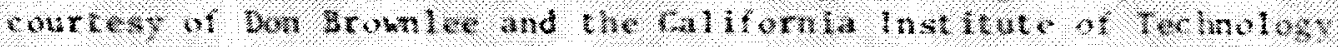

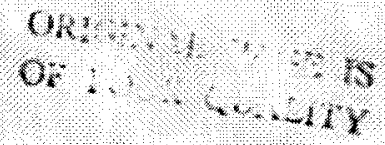




\section{EARTH-BASED OBSERVATIOAS}

A proposed network of ground-based ubservers representing seven separate disciplines is outlined in section IX of this report. One area of study (meteoroids and meteor studies) may require special observation types and techniques and is discussed here.

The orionid and Eta Aquarid meteor showers are believed to be debris from Comet Halley. They probably are samples from Halley released at distinctly different eras in its evolutionary history. Although the showers are annual, Yeomans has pointed out that in October 1985 and May 1986 the Earth will pass close to the conet's orbit and not far from the conet's nucleus. Enhanced activity ay occur at these times, but ouing to the long orbital period of Halley there are few observations to assist in aking predictions. Yeteor observations potentially can provide direct information on the chemistry, density, and size distribution of Halley debris. Infortunately meteor study is an extinct science in the linited states and production of nes data by professional researchers is possible only from the active groups in Canada, Europe, and the soviet inion. However, active amateur observing groups exist world wide.

A valuable aspect of meteor work is the potentiai to determine composition and density. Infortunately, conversion of weteor data to these parameters has never been convincingly deanstrated to desirable levels of accuracy. Iaprovement in this dismal situation will require renewed efforts. Observations which are routine and $f$ it in with the Halley hatch program are visual meteor counts during the showers. Horlduide groups of trained observers could be coordinated to provide accurate meteor rates during the years 1983 to 1987 . This could provide valuable data for the distribution of meteoroids within the two Halley st reams.

Actual collection or direct detection of particles in the Halley meteor streams is possible, in principle, by several techniques. Particles with appropriate release points and ratios of radiation pressure force to gravity can hit a spacecraft in the comet's orbit plane outside of the comet's orbital ei i ipse. In this regard it is at least conceivable that a detector on some interplanetary probe could detect impacts from particle's released during the present Halley apparition. Detection of particles released from come kohoutek was made by the Max Planck Institute micrometeoroid impact sensor on the Hros I! spacecratt. In this case the particles were released at a significant solar distance and had radiation pressure force to gravity ratios of unity.

Near the Earth, several orbital and atmospheric experiments are possible. Nondestructive collection is not practical owing to high impact velocities, but capture cell collectors can be used to obtain vaporized and recondensed Halley macerial for bulk elemental and isotopic analysis. Positive identification of particles within the shower could be accomplished by accurate measurement of impact velocity. Detection of small particles without sample return is possible using micrometeoroid impact sensors of the type routinely used on interplanetary missions. A major uncertainty with orbital detection or collection is the possibility that meteor showers do not contain small particles. Yicron-sized 
particles rapidly change orbits because of Poynting-Robertson drag, and they quickly evolve out of the main showers of visual meteors. Micron-sized particles have not been detected in annual meteor showers by orbiting micrometeoroid sensors, but the claim has been made that showers were detected for $0.1-\mu \mathrm{m}$ particles by the Heteoroid Technology Satellite.

The IHW advocates and can play a limited role in coordinating cometary dust studies frum deep space and earth orbital spacecraft, during stratospheric meteoroid collections by aircraft and mesospheric detection by rocketborne instrumentation. However, the IHW can take a more direct role in organizing and coordinating a global network of visual meteor observers. Worldwide groups of trained observers (both professional and amateur) could be coordinated to provide accurate meteor rates during the 1983-1937 interval. These observations could provide valuable data on the distribution of meteoroids within the two Halley streams. 
IX. GROUND-BASED OBSERVING NETS

\section{A. LARGE-SCALE PHENOMEIA}

\section{Scientific Objectives}

The prime objective is to investigate the comet solar wind interaction and to provide a calibration of solar wind properties deduced from comets. He are beginning to understand the correlation between solar wind properties and cometary phenomena such as tail orientations, disconnection events, brightness fluctuations and other transient events. Thus, we can infer solar wind properties at the comet by a study of wide-angle images obtained simultaneously with spacecraft in situ measurements. The latter include the solar wind speed, density, and magnetic field as well as the same quantities in the mixed cometary/solar vind plasma at the time of closest approach.

Assuming the re are one or more space missions with inaging capability at some distance from the Earth it should be possible to obtain steroscopic views of the comet using ground-based and spacecraft cruise-phase images, and to infer the comet's three-dimensional structure. While a spacecraft is far from Halley's Comet, both before and after flyby, the imaging system could obtain views similar in scale to those obtained by Earth-based, wide-angle cameras. The ground-based imaging network should be spread in latitude and longitude in a manner such that the comet will always be observable from several sites, at least one of which is likely to have satisfactory weather conditions. We would be able to provide a ground-based photograph close in time to each spacecraft image of the comet.

The pairs of images would contain considerable information on the three-dimensional structure of the comet and its temporal behavior. At the time of flyby, a spacecraft would be able to obtain images of the comet from a large number of viewing angles. As a result, it may be possible to construct a three-dimensional model of the comet using techniques similar to computed tomography as developed for medical $X$-ray work and modified by us for this application. The additional wide-angle views from Earth would serve as a control to separate temporal changes from spatial changes.

Also assuming there are space missions to Halley, it will be possible to provide extensive coverage of the large-scale structure of the coma and tail of the comet during flyby. While the spacecraft is carrying out its brief but crucial in situ observations, it will be constrained to studies of fine-scale structure of limited portions of the comet. The ground-based imaging network will ensure simultaneous coverage of the entire comet, thus providing a record of transient phenomena that will greatly assist in the interpretation of the in situ data.

\section{Technique for Achieving the objectives}

In order to achieve the objectives of this phase of the Halley Watch, there must be a network of observatories, with wide-field imaging capabilities, having one dominant goal. At least one participating observatory must be able to obtain a wide angle image of Halley at any and all times during spacecraft encounters and during prime study periods. The latter are primarily the months of November 1985 and April 1986, when Halley is closest to Earth and reasonable spatial scale 
can be obtained. Images should also be obtained on selected days in cooperation with other observing disciplines. To achieve this objective requires a network of observatories as uniformly distributed in longitude as the presence of land masses will permit, as near the equator as possible, and in both Northern and Southern Hemispheres.

Since photographic data will be obtained from many different locations under a variety of observing conditions, it will be necessary to give the utmost attention to the calibration of the individual images. To begin, all images will be collected on Kodak I Ia- 0 photographic plates, or their equivalent. The calibration method to be used will be an outgrowth of the technique proposed by John Kormendy. 2 Use of his method requires obtaining the brightness profile of standard stars made with the same telescope/film combinations that will be used for the Comet Halley observations. The shapes of the standard stars will determine the relative intensity transfer and their known magnitudes, along with photoelectric sky background measures, and will provide the absolute zero point of the intensity scale. Errors of the order of $<0.05 \mathrm{mag}$ are expected. The procedure must be applied to each telescope in the network since the measured shape of the star profiles will depend on the optical condition within that telescope. We will request that each observatory provide us with a sequence of plates of some large, wel1studied, extended objects (for example M31 andior '181) at the time of Comet Halley flyby, so that we may calibrate their telescopes in the conditions that the comet images were obtained. In the event the original plates are not sent to us, the observatory will be asked to make copies on Kodak Fine Grain Positive (7302), or equivalent.

Another problem that must be attacked is the combining of images obtained from telescopes with different plate scales and optical distortions. The choice of digitizing step size must be adequate to provide sufficient digitizing resolution so that the resampling necessary to remove distortion and match plate scales will not degrade the resolution of the final product.

\section{Setting up the Net}

A network of 18 ohservatories was set up in less than a year to observe Comet Kohoutek in 1973-1974. Interesting results were obtained even without the standardization and calibration procedures proposed here.

Preliminary inquiries have found more than 30 observatories willing to participate in a Large Scale Phenomena observing network. It is readily apparent that the required coverage in both longitude and latitude is met rather we 11.

4. The Discipline Specialist and Data Handling

a. Results Expected. The resulting raw data from the network will be a large number of calibrated photographic plates. It is proposed that these plates be copied onto a transfer emulsion for subseaucnt study by the network team, the original plates being returned to the observatories. The data will be used in several different ways by the team: 
(1) Working pictures will be provided to the Halley Watch as quickly as possible to be used by all other investigator teams as needed.

(2) The best time sequences in the data set will be digitized and computer-processed to a uniform intensity level and scale. These images will then be combined into motion picture format for the study of the time history of cometary activity by the network team and others.

(3) The best 1000 frames will be published as an at las of Halley's Comet, with time development taken into consideration.

b. Data Analysis P1ans. In order to obtain effective use of the images from the Comet Halley network, it is necessary to develop a detailed data analysis plan. This plan, like Comet Haliey, must be a dynamic, evolving entity that will lead to the maximum scientific return with the minimum effort and expense.

The data analysis can be divided into two major parts: data reduction/ calibration and scientific analysis. The data reduction/calibration has several steps that mist be accomplished. These are collecting and cataloging the original plates and/or first film copies, selecting and digitizing the necessary images and associated calibration data, converting the photographic density/transmission measures to relative intensity, and matching all images to a common plate scale and background remosal.

The scientific analysis part will include construction of mosaics of several images to obtain the total comet image, time sequences of disruption view angles, and possibly a third view angle in order to provide three-dimensional information, contour mapping of all or part of the comet tail, total emission measurement, etc.

c. Volume of Data. There are currently about 25 observatories with widefield type telescopes located around the world that have given a positive indication toward cooperation in the Comet Halley network. If we can expect a twomonth period before and after perihelion with $60 \%$ clear sky and two good plates per clear night, there will be 25 observatories $\times 4$ months $\times 30$ nights/month $\times 0.6$ clear $\times 2$ plates $/$ night $=3600$ plates with a field of view of $5 \times 5^{\circ}$. If all of these images were digitized with a 7 arc-sec step, there would be $2570 \times 2570=$ $6 \times 10^{6}$ pixels per plate. At a magnetic tape packing density of 6250 BPI it will be possible to store 6 images/tape of the size mentioned above or the complete 3600 images on 180 tapes. We estimate that about one-third of the images will be digitized, thus reducing the raw data 1 ibrary to about 60 tapes or a small number of video discs. With processed images and backup tapes the library may reach 300 tapes.

d. Resources Needed. In order to carry out the data reduction and scientific analysis as described, access to several major hardware and software items will be required. These items include the following: 
(1) Microdensitometer of sufficient speed and resolution to provide a timely and accurate rendition of the photographic density/transmission.

(2) Sufficient computer power and data base storage to catalog the digitized imagery and perform the necessary calibration effort.

(3) Sufficient interactive computer power to permit the astronomers to perform and observe the scientific analysis of the comet imagery as it is carried out.

(4) Sufficient hard copy facilities that can provide both inage playback, graphical analysis, and numerical results.

(5) Sufficient dark room facilities and expertise to produce the many film products required in both the data reduction and scientific analys is phases.

e. Sumary of Output. The output of the network will be (1) working, wide-angle images made during critical times provided rapidly to a Halley mission project; (2) movies of the best sequences of observations, made from calibrated, fully processed images; (3) an atlas of the best quality, calibrated, processed images; and, (4) scientific papers by the scientific team on results of in-depth analysis of the observations.

\section{B. NEAR-NUCLEUS STUDIES \\ 1. Goals and Techniques}

Observations of the near-nucleus region, the inner coma, or the false nucleus as Opik calls it, provide the most important data about the general activity of the comet, about its spin axis, and about the distribution and variation of specific active areas on the nucleus itself. Such observations, coupled with diameters of halos, when observable, lead to the determination of the period of rotation of the nucleus and, over long time intervals, to possible variation in the spin period.

Visual measures of near-nucleus phenomena such as jets, fans, globular extrusions, multiple nuclei, asymmetries in the coma, diameter of the nucleus, and photometry of nucleus brightness and color are all important in reconstructing the structure of the nucleus surface and its activity. Telescopes of 15-cm aperture and greater can be useful to these ends. Drawings are extremely valuable. Long focal-length telescopes used photographically or with image tubes can provide permanent records of these phenomena. In all cases complete records of times, equipment used and sky conditions are vital.

Color filters, polarization devices, and objective dispersion spectrographs have been little used in the analysis of nuclear activity but show great promise of adding extremely important results when theoretical and analytical techniques become more sophisticated in the study of the nucleus itself. The application of high-dispersion spectroscopy in and across the coma is discussed elsewhere. But most phenomena of the coma and some of the tail can be tracked back in time to the observable near-nucleus activity. 
Photographs taken for astrometric purposes always have the shortest exposure possible for a vell-developed image. This minimizes both image motion and any question about the location of the image centroid. Photographs taken to study large-scale phenowena necessarily have large-scale and very long exposure to bring out structures of low surface brightness in the long comet tails. Neither technique serves to inage the relatively small, woderately bright structures of the inner coma.

In principle, wodern imaging detectors such as the CCD (charge coupled device) have sufficient dynamic range to properly image both nucleus and coma. Their fornat is limited in size, however, making the availability of a sufficient number of astroweric reference stars unlikely. Further, these devices are still expensive, somewhat experimental, and available at only a few observatories. Therefore, it seems necessary to create a small network of observers for near-nucleus studies.

Properly exposed photographs would provide an adequate base for a minimal study of the inner coma. Proper use of filters to separate gas and dust will indicate if their mixture within the coma structures is the same as that in the wre uniform background. Use of imaging detectors such as the CCD would be a far better procedure. The exposure times required would be 2 orders of magnitude less. This not only saves telescope time but should result in pictures of far higher resolution, with smear effects due to poor guiding and seeing effects greatly minimized. Further, a quantitative study of any composition differences in the structures would be feasible, to the extent that they have species emitting within the wavelength range of the detector.

\section{The Discipline Specialist and the Net}

For studies requiring continuous coverage, such as measurement of rotation, the near-nucleus network will have to have good coverage in longitude. For simple photographic work, a second, longer exposure by members of the astrometric network would suffice. For imaging detector studies, the discipline specialist will have to prevail upon those observatories which have the detectors. If there are major gaps in the network, it may be necessary to supply equipment to fill then. As noted earlier, visual drawings by experienced amateurs can be of value in the study of the inner coma. These can give the kind of continuous coverage otherwise difficult to obtain. Angular scale and orientation calibration is, of course, critical.

Quanticative results from imaging detectors require considerable computer processing. Most facilities having such detectors will be equipped to reduce their own data, but the discipline specialist may have to process data taken by supplied equipment.

\section{SPECTROSCOPY \\ 1. Goals and Techniques}

The goals of all spectroscopic studies are very basic to understanding Halley and all comets. We wish to know the composition, physical state, origin, and loss of the molecules present in the nucleus, coma, and tail that have spectroscopic signatures available for study. 
Spectrophotometry using the varlous high-speed scanning technique; is usually limited to spectral resolution of no more than about $5 \AA$ throughout the spectral range $0.3-1.0 \mu \mathrm{m}$. For many studies this is inadequate, and in some cases where it is adequate, an observatory may have a classical spectrograph available but not one of the new electronfic detection systems.

Among the many important spectroscopic studies that can be undertaken from the ground on Halley or any bright comet, the following certainly must be included. There are many unidentified features in cometary spectra, and their analysis typically requires a spectral resolution of about $0.1 \mathrm{~A}$. Some features may prove to be new bands of known molecules, but some are probably new molecules (e.g., the feature at $3257 \AA$ in Kohoutek).

The excitation mechanism of most cometary vibronic bands appears to be resonance fluorescence, in which molecules in the ground state and present in low number densities are directly excited by sunlight and then cascade back to the ground state. The solar flux available for excitation depends upon the heliocentric velocity of the comet, wherein the doppler effect shifts the spectral features relative to the Fraunhofer spectrum of the Sun (the Swings effect). In the inner coma the distribution of intensity among rotational lines may be wodified by collisions (the Malaise effect) and further modified by differential velocities within the coma (the Greenstein effect). The study of each of these effects has been carried out on only one or a few comets (obviously not Halley) and only for one or a few bands. Each requires resolution of individual rotational lines in the bands, and the latter two effects especially require good spatial resolution as well. Therefore, study of these effects requires a large telescope.

Another spectroscopic study of importance, requiring high spectral resolution, is that of isotope bands. The $1-0$ band of $c^{13} c^{12}$ has probably been observed in at least two comets, but it is badly blended with a high overtone of $\mathrm{NH}_{2}$. Perhaps a Fabry-Perot interferometer could be used on a large telescope for this study. Thought should be given to the study of other bands as well. A CCD detector used with a high-dispersion spectrograph to observe bands at longer wavelengths of fers another possibility.

A smaller telescope and spectrograph that offers good spatial resolution, but only modest spectral resolution, can be used to study the brightness profiles of the various molecular bands as a function of nucleus distance. The scale lengths for creation and loss of the observed radicals, many of which are still unknown for any comet, can be derfued in this fashion. If the scale lengths are the result of a pure photo process, they should vary directly as the square of heliocentric distance. Otherwise anything is possible. Therefore, Halley needs to be studied all along its orbit.

Absolute production rates can be derived spectroscopically, of course, just as they can from photometry (see Section IX-D). If this is done using photographic detection, there will be the usual loss in precision inherent in the photographic process. Using a photomultiplier or any of the modern detection schemes, there is no loss except possibly in speed, and there may be a gain in precision.

Halley's ion tail will have comparatively low surface brightness but may extend to a length of many degrees even at this unfavorable apparition. Small but fast telescopes or cameras can be used with an objective prism or an objective 
grating to obtain quantitative photometric data in the 1 ight of each tail ion. Most such devices will have only photographic detection available, but perhaps a few can be equipped with modern two-dimensional silicon arrays of various types (CCD, CID, ISIT).

\section{New Operations}

A coordinated program in spectroscopy has to be limited to the times when Comet Halley is brighter than $m=14$ and should be concentrated during the period when it is brighter than $m=10$. High spectral resolution will be limited to $m \leq 6$. These considerations yield the following schedule:

互

$\leq 14$

$\leq 10$

$\leq 6$

$$
\begin{aligned}
& \text { Period } \\
& 8 / 10 / 85-11 / 1 / 86 \text { (16 months) } \\
& 10 / 15 / 85-7 / 10 / 86 \text { (9 months) } \\
& 12 / 1 / C j-5 / 1 / 86 \quad \text { (5 months) }
\end{aligned}
$$

From January 18 through February 26, 1986, Halley will be within $30^{\circ}$ elongation from the Sun and impossible to observe at most telescopes. This will also be the case for two months beginning in mid-August of 1986 .

The intense period of operations will therefore be of less than four months' duration. It will probably take place mostly during the bright of the Moon, since dark time will be demanded by other Halley observers for photometry and photography. However, provision should be made for a rapid shift to spectroscopy on those dark nights when cirrus clouds, etc., make photometry difficult or impossible. There will be great pressure on southern observatories where better weather can be expected during the prime period and where Halley will be tigher in the sky and available for more dark hours for most of that time.

\section{The Discipline Specialist and iata Management}

a. Reduction of Observations. In order to encourage a fast reduction of observations on a homogeneous basis, it is proposed to offer all observers the services of a Reduction Center, where the spectrograms would be put on tapes by using a digital system coupled to a microdensitometer with 2-dimensional scanning. The plates would be sent to the Center and would be promptly returned (2 to 6 weeks). The observer would then receive, at his choice, either a copy of the magnetic tape, or data already printed and reduced. The tape would be available promptly but the reduced data would be delayed by a few weeks or months, depending on the work load of the Center. The observer would of course keep his proprietary rights to the data (for example, for six months after he has received either the tape or the reduced data). The agreement with the discipline specialist might vary from observer to observer. In exchange for its service, the Center would then of fer access of all data to the scientific community after a reasonable delay. In particular, it would publish an Atlas of representative spectra of Comet Halley, with reduced microdensitometer profiles of significance and scale lengths of representative brightness profiles. 
b. Processing Data. The discipline specialist and his spectroscopic data reduction equipment could be located anywhere, but few if any astronomy departments could handle the data load expected without adding some equipment. It is estimated that an upper limit of 1000 spectrograms (probably much less) would be collected. Since the data should be processed in a few months, the existing hardware in most university departments would require modifications and/or augmentation.

\section{PHOTOMETRY, SPECTROPHOTOMETRY AND POLARIMETRY \\ 1. Goals}

The general aim of these disciplines as applied to cometary research is to acquire quantitative information on the composition and physical state of both volatile and non-volatile components of comets and on the astrophysical processes which occur in both heads and tails.

Far more can be learned from the systematic study of the variations in a comet with heliocentric distance, nuclear rotation, solar activity, etc., than from a few random measurements. From the variation in coma abundances with heliocentric distance we can place constraints on the vaporization process of the cometary nucleus and the source of the observed species. By studying the variation of the continuum color, intensity, and polarization with heliocentric distance we can study how the grain component of the coma changes. The concomitant change in scattering angle as the heliocentric distance changes will provide information about the scattering function of the grains and hence will place constraints on their physical nature.

By looking at similar variations on shorter time scales we can determine the nuclear rotation rate, discern spatial variations of the nuclear surface, and with some luck obtain, for the first time, detailed knowledge of the outbursts which many comets undergo.

Observations made simultaneously with those by other disciplines at other wavelengths will enable us to relate the abundances of optically observed species to the abundances of species observed (or not observed!) at other wavelengths and thus obtain insight into possible parent molecules. Another interesting point to be investigated is the relationship between the optical reflection spectrum of the solid particles, the thermal infrared emission spectrum of the solid particles, and the strength of the infrared silicate emission feature.

\section{Proposed Observing Network}

Comet Halley will be south of $-10^{\circ}$ declination from early February until mid-May 1986. The observational coverage necessary for the photometric disciplines will require a number of observatories in both hemispheres, well spaced in longitude in order to obtain the continuous observations needed for studies of rotation and outbursts. Having observatories in both hemispheres will help obviate weather problems as well as the necessity of observing at large air masses near the horizon. Because of the substantial differences between the techniques of photometry, spectrophotometry, and polarimetry, it is necessary to considered separately the questions of observing networks and procedures. 
a. Filter Photometry. Narrow-band filter photometry is an advantageous cechnique for cometary observations for the following reasons: (1) it requires only relatively simple equipment available at many observatorles; (2) it allows observation of the whole cona, or at least a large fraction of it, at once; and (3) It is relatively sensitive and can be used for faint come:s, i.e., at large neliucentric distances and for on small telescopes.

In order to achieve all of the science goals, it will be necessary to have a substantial network, all observers in the network being equipped with identical sets of filters. Experience with photometry of past comets indicates that narrow band photometry can, without too much effort, be carried out to visual magnitude 13 or 14 on a $75-\mathrm{cm}$ telescope. Telescopes of $60-$ to $90-\mathrm{cm}$ aperture are quite widespread so that it is realistic to set up a complete network for observations to magnitude 14.* Ideally we would like to end up with a total network of 25 to 30 observatories with at least half of these in the southern hemisphere. For observations fainter than $14^{\mathrm{m}}$, we propose a much smaller network of perhaps a half-dozen large telescopes $(\geq 150 \mathrm{~cm}$ ).

The equipment for this effort is modest and we can presurs that all of the observatories are already equipped with photoelectric photometers. It wi'l be necessary, however, to provide filter sets for most of these observatories. The IAU Commission 15 filter sets will provide a good start but this will not be adequate for a comprehensive Halley Watch. Although the IAU filters committee is planning to retain 10 to 15 sets of filters for loan on special projects (presumably the Halley Watch might be such a project), this number of sets would be marginal for a really thorough Halley Watch. For this reason, it would be advisable for the Halley Watch to purchase additional sets of filters simultaneously with the purchase by the IAU committee, i.e., in early FY-81. The Halley Watch discipline specialist should also investigate the purchase of other filters to isolate features not covered by the IAU set (such as $\mathrm{CO}^{+}, \mathrm{OH}$ and $\mathrm{NH}$ ).

Although the observing schedule must be coordinated with the other disciplines, a good mode of operation would be to designate groups of two or three days spaced at intervals of 0.1 in $\log r_{H}$ for $r_{H} \gtrsim 2.0 \mathrm{AU}$ and at intervals of 0.05 in $\log r_{H}$ for $\mathrm{rH}_{\mathrm{H}} \succsim 2.0 \mathrm{AU}$. For $r_{\mathrm{H}} \succsim 2.0 \mathrm{AU}$, the half-dozen large telescopes would be asked to attempt one set of observations in each of the designated periods. For $r_{H} \lesssim 2.0$ $\mathrm{AU}$, all the small telescopes (total of 25 to 30 ) would be asked to obtain as much data as possible in the designated periods, thus enabling studies at high time resolution and better coincidences ith observers at other wavelengths.

Because of the 1 imited period during which Halley would be visible from a single site, a typical "night" of observing would only last 4 or 5 hours, including observations of standard stars and extinction stars. Observing procedures would probably be adapted from those expected to be produced by the IAJ filters committee. The biggest problem is likely to be changing the observing habits of those volunteers who are prisarily stellar photometrists.

b. Spectrophotometry. This technique provides an important complement to filter photometry. It wili be much more difficult to implement because there

*Halley can be expected to be brighter than 14 th magnitude for a full year. 
are reiatively fewer observatories equipped for this sort of observation and the requisite telescope time is more difficult to obtain. Proper advance warning and "consciousness raising" of observatory directors way help to solve this problem.

We would aim for a rather small network of about 5 to 10 telescopes with gocd spectrophotometric equipment capable of scanning the entire cometary spectrum, from the atmospheric cutoff in the ultraviolet to about 1 micron. We would also aim for equipment yielding a spectral resolution of $10 \AA$ or betcer. In terms of observing procedures, we would leave this largely up to the individual observers although we would provide them with the lists of standard stars being used in the filter photometry programs with the suggestion that they at least observe these stars even if they did not use them as standards for flux calibration.

He would ask each member of this network to attempt to obtain one goud spactral scan (complete wavelength range) of the comet in each of the periods during which the photometry network was active. The observer could thus work the observations of Comet Halley into other observing programs, making it possible to obtain large telescon, time at more frequent intervals than would otherwise be the case.

c. Polarimetry. Although nearly anv telescope can be used i.r polarimetric measurements utilizing a sheet polarizer and a photographic plate, the results are likely to be of relatively little value. Several groups, however, have modern polarimetric apparatus which could be used to study comets. These groups should all be requested to attempt observations during some or all of the designated observing periods. Because the number of groups are small, the polarimetry effort must be small. A key role of the discipline specialist will be to suggest standa dized bandpasses to isolate the cometery continuum.

\section{The Discipline Specialist and Dara Management}

In most instances, a portion of the data analysis would be carried out by the individual cbservers, because they are the ones most familiar with the details of local instrumentation. Typically a photometrist would supply apparent magnitudes (instrumental system) outside the atmosphere, a spectrophotometrist would supply spectral scans corrected to outside the atmosphere (and possibly on an absolute scale), and a polarimetrist would supply percent polarizations and position angles. In some instances, the observers would only be able to provide raw data and the discipline specialist must be prepared to reduce raw data in these relatively few instances. In most cases, however, the data reduction demands should not be major, consisting primarily of the conversion from the various instrumental systems to an absolute system. The discipline speciaiist would also be expected to determine the quality of all data submitted (e.g., by looking at the precision of extinction fits and the interagreement among standard stars).

The discipline chief should have access to some computing facility with a reasonably large mass storage device such as discs, but the demands are not large and most institulions would already have adequate, although perhaps not optimal, facilities. An interactive terminal, for example, would be desirable but not 
essential. The wost difficult requirement would be an ability to read the various media in wich data vill arrive.

Standardization of data formats and transmission of the data to the of fice of the IHW leader vould also be the responsibility of the discipline specialist. This portion of the job would be simplified by providing direct access to a central computer at the Ith leader's institution, but the data analysis phases would be better carried out on a computer at the discipline specialist's institution.

\section{E. RADIO rCIENCES \\ 1. Coals}

Thiee genelal radio science techniques have been applied to cosets, of which only one has achieved any significant success. This successful technique is the study of discrete wicrowave molecular emission lines. For example, in many conets the OH moiecule has been seen in absorption (presumably against the galactic background rather than any cometary contiruum) and in emission at $18 \mathrm{~cm}$. Searches for a microwave continuum have shown mixed, marginal resulrs. Radar studies have had uniforaly negative results to date. The radio spectroscopic goals should include:

OH studies for understanding pump mechanisas and for obtaining line widths.

CH and CN studies for optical comparisons.

Detection of unidentified lines which may point to important, unknown species not observed in interstellar clouds.

Detection of $\mathrm{H}_{2} \mathrm{O}, \mathrm{NH}_{3}, \mathrm{HCN}$ and other large molecules which may be parent molecules of cometary radicals.

Detection of cometary amino acids.

\section{Techniques}

Molecular observations of comets with radio telescopes have several advantages:

(1) With radomes and sun-screens, one can observe quite close to the Sun.

(2) Microwave observations have the resolution necessary to determine comet coma line widths (OH lines are typically $2.5 \mathrm{~km} \mathrm{~s}^{-1}$ ). Yoreover, the high resolution allows accurate frequency determinations which can facilitate identification of any new spicies.

(3) Microwave spectra are simple rotational transitions and the region is not cluttered by a superposition of electronic, vibrational, and rotational transitions.

(4) Microwave receiver system: are tunable over a wide range of frequencies which allow a particular telescope to probe many suspected molecular constituents of comets. 
(5) Hicrowave observations allow probing of the nucleus of dusty conets (Halley is one); In fact, one ay need a hot, dusty conet for production of radio species if past observations are any indication.

On the other hand, radiv observations have some disadvantages toc:

(1) The radio antenna temperatures detected in conets (HCN, OH, $\mathrm{CH}_{3} \mathrm{CA}$, $\mathrm{H}_{2}$ ) ) are generally near the detection liats of the equipant. For example, one needs a production rate Qspecies $>10^{28} s^{-1}$ to be able to detect most moiecular species.

(2) Bean dilution for comets can be severe. For exaple, if one assumes a species scale length of $5 \times 10^{4} \mathrm{~km}$, then the distance at wich the source vould just fill the bean of the antenna for a given telescope observing a particular species is indicated in the following:

\begin{tabular}{|c|c|c|c|c|}
\hline Telescope & $f(\mathrm{CHz})$ & HPBW & Delta (AU) & Species \\
\hline nRAO $140^{\circ}$ & 4.8 & $6.2^{\prime}$ & 0.37 & $\mathrm{OH}, \mathrm{H}_{2} \mathrm{CO}$ \\
\hline haystack $120^{\circ}$ & 22.0 & $1.4^{\circ}$ & 1.64 & $\mathrm{H}_{2} \mathrm{O}, \mathrm{NH}_{3}$ \\
\hline MRAO $36^{\prime}$ & 90.0 & $1.3^{\prime}$ & 1.77 & $\mathrm{HCN}, \mathrm{HCO}^{+}$ \\
\hline
\end{tabular}

In view of the above, it will be importarit to accurately match available radio receivers and spectrometers to the conetary position in order to optinize the detection possibilities.

\section{Net Operation}

A major problem will be acquisition of sufficient observing tire. We need to have radio observatories devote a large amount of telescope tine for comet Halley throughout much of its apparition. Observatories gear up for targets of opportunity to varying degrees. Radio observations need to be keyed to pre- and post-perihelion distances to better understand the physical processes in conets. For example, escept for $\mathrm{OH}$, no radio molecules have been seen in comets that have had perihelia $\geq 1$ AU. The radio network should be operated at appropriate points in the OH pumping cycle and near perihelion for molecules and continuum searches. Horeover, radic mapping of nolecular species as a function of distance from the comet will require telescope time near the closest approach to Earth in order to minimize beam dilution problems.

We need to get the directors of observatories involved as soon as possible. Direc-ars need to know that a definite comitment of telescope time and observatory resources (computers, in particular) is needed. When radio observatories commit themselves to support the IHH, proposals should be solicited by the major radio observatories in order to notify the astronomical community that the observatory backs the IHW concept and in order to allow enough time for the discipline specialist to check against duplication of effort and holes in coverage. 


\section{Discipline Specialist and Data Management}

The discipline specialist will be responsible for reviewing all radio data. All data subnitted to hin shall be fully reduced with appropriate telescopic and atmospheric corrections applied. Because of the esoteric nature of radio data, the discipline specialist should be responsible for screening all archival data. For exanple, an appropriate standard for Inw acceptance would be publication of the data by a recognized journal. In arginal cases, the discipline specialist ay want to consu't with other experienced radio observers and thereby assign a statistical weipitt to the dacz.

The discipline specialist must provide a satisfactory solution to the ephemeris software problea. Each radio telescope system is unique in its tracking facilities and its capability to observe a solar syster object (fast woving source). The Ind softuare analyst could distribute a well-documented copy of a FORTRAr comet ephemeris progran to radio observatories. This standard progran should have the format and corrections included that the planets have in the Arerican Ephereris and Nautical Almanac. Most observatories have adapted co this in the past. Having the IHW ephemeris program will allow the observatory to adapt it to a particular telescope's unique needs. Hoveover, any unexpected orbital perturbation can be accomodated quickly if observatories have their om progran version.

Finally, the discipline specialist will monitor planned radio observations and available equipment. He vill coordinate equipment needs with available funding before the network is activated and coordinate ongoing radio observations with observations in other parts of the spectrum after the network is activated.

\section{F. RADIOAETRY \\ 1. Goals and Techniques}

Experience with observations of six comets in the last decade has shown that coma, tail, and antitail measurements with fixed diaphragms and infrared beam switching techniques lead to reproducible results which can be obtained by a number of observatories. These allow quantitative determination of the solar energy reflected in the visual wavelengths and the energy and temperature of the thermal radiation. When small silicate particles are present, the 10- and 20-micron silicate features are in evidence.

Diaphragm photometry (with different sized diaphragms) of the coma can be corrected for the Earth-comet distance. In a given diaphragm, the flux is inversely proportional to the Earth-comet distance. When the diaphragm size is varied the flux is proportional to the diaphragm diameter (as would be expected from the continuity equation for dust ejected from the nucleus and traveling with constant speed). The relation holds for diaphragm diameters of a few seconds to a few minutes of arc.

Previous comets have show a number of interesting features including:

(1) The presence of silicate grains of small diameter (less than one micron) in the comae and tails of dusty comets. 
(2) The presence of large grains in the antitail of comet Kohoutek (the silicate signature disappeared).

(3) A change in the strength of the silicate signature and its complete absence in the comae of some comets with predoninately ion tails.

(4) The strongly forward scattering optical phase function characteristic of dielectric particles (Comet West).

(5) The increase in the radiated pover when the nucleus fragments and produces wore grains for the same nuclear mass.

(6) Differing comet albedos associated with the strength of the silicate feature and therefcre presumably the mean grain size.

Many observatories equipped with wobbling secondary mirrors can make the required easurements. Telescopes of aperture greater than $60 \mathrm{~cm}$ are adequate. For exampie, with a 90-cm telescope of $\mathrm{f} / 11$, the projected beam on the coma is 26 arc seconds for a 1-millimeter diaphragm. Telescopes with longer focal lengths project corespondingly smaller beams.

Of particular importance in the case of Halley is the capability of these observations to produce data at small elongations. Previous experience has shown that it is possible to obtain excellent data at all radiometric wavelengths at elongations of a few degrees from the Sun, so Halley could be completely covered. In these observations, the beam-switching capability is required in order to cancel the surface brightness of the coronal aureale.

\section{Proposed Observational Net}

More than a dozen observatories over a wide geographic area have previously undertaken cometary radiometric studies or have the necessary facilities to make such observations. If all of these facilities (and perhaps others expected to beccme operative) could be persuaded to make observations, excellent geographical and temporal coverage could be obtained.

In addition to the ground-based observatories, the NASA C141 Kuiper Observatory could perform infrared photometry and spectrophotometry covering many of the regions between the windows for the ground-based observations. This would be of great importance in determining the exact shape of the 10- and 20-micron emission features. Special atcention to the wavelength regions inaccessible from the ground might lead to the discovery of other diagnostic spectral signatures in the infrared.

\section{The Discipline Specialist and Net Cperations}

The coordination of data acquisition is important, but the Halley Watch must recognize the proprietary rights of the individual investigators to analyze their own observations. It should be possible after individuals have studied their results and published in the normal way to assemble all the data and produce a cooperative, chronological publication and overall analysis. 


\section{G. ASTROMETRY}

1. Goais

The astrometry network will provide the accurate astrometric and nucleus observations of Comet halley that are required for orbit and ephemeris computations, for dynamical modeling of the comet's outgassing rocket effect, and for estimates of the dianeter of the comet's nucleus.

The astrometric observations of the comet will be made throughout the cometary apparition and will be employed to continually update the orbit and ephemeris of the comet - a function that is important for supporting observations within the other disciplines and critically important for supporting a Halley flythrough mission. Astrometric observations will also allow refinements in the existing model for this comet's nongravitational forces and the characterization of the comet's spin axis orientation and spin direction.

Attempts will be made to observe the bare nucleus of Comet Halley when the comet is at large heliocentric distances and hence inactive. Assuming that the light from Comet Halley at large heliocentric distances is due only to reflected sunlight, the comet's photometric cross section (AS) can be determined. Using the technique of Delseme and $\mathrm{Kud}^{3}$. One can compute the vaporization cross section (1-A)S from the water production rate. Hence the bond aibedo (A) and cross sectional area (S) are determined and the comet's diameter can be inferred.

The astrometry network's observations will also be used to define the time scale and amplitude of the comet's brightness at large heliocentric distances.

\section{Techniques}

a. Astrometric Positions. The astrometry network would be staffed by experienced observers who have access to astrographic telescopes, on-site measuring engines and appropriate star catalogs. Observations of the comet should guide on the comet's motion to obtain nearly stellar images, hence minimizing centerof-light/center-of-mass offsets. Each observer should take at least two plates per evening to identify weak images and proper'y identify the comet by comparing the observed and computed motion of the image. To facilitate plate rejuctions, each observer should be equipped with the appropriate star catalogs for each section of the comet's path through the constellations. At least 5 reference stars should be used in the reduction procedure and the astrometric cometary positions should be referred to the mean equator and equinox of 1950.0. Astrometric positions should be transmitted to the orbit determination center by telegram or TELEX using tiec standard IAU circular code or by phone.

b. Nucleus Observations. Observations made to observe the bare nucleus of Comet Halley should be made at the greatest possible heliocentric distance both pre- and post-perihelion. The observational biases due to aperture and $f$ ratio effects should be minimized by using a single instrument for long series of observations. Each separate observation series should overlap the other series 
for proper calibration between series. Each observer should plan to use the standardized techniques agreed upon by members of the astrometry network. The overwhelming concern for this type of observation is the spurious light introduced by dust surrounding the comet and any light originating with the comet. Both effects would lead to an estimate of the comet's diameter that was too large. To insure that bare nucleus observations are made and that the measured magnitudes are due only to reflected sunlight, the measured apparent magnitudes should:

(1) Follow a $\mathrm{r}^{-2}$ law.

(2) Be symbetrical with respect to perihelion.

(3) Display a phase lau (this may be difficult to detect because the change in phase angle at large $r$ is not great).

(4) Have stellar images that are independent of exposure $t$ ime.

Photometric data taken at large heliocentric distances where there is no evidence of emission should be subjected to the same four criteria as photographic data taken for astrometric purposes. Results either from filter photometry or spectrophotometry (as discussed in section A) that meet the criteria can firnish precision values for the photometric cross section (AS) as discussed previously.

other opportunities to investigate the nucleus from the ground would occur if the comet occults a star or if the comet is large enough and close enough to return a radar signal. Although the event would be improbable, a search should be made to identify stars that may be occulted. Even if the star passed close to the apparent location of the nucleus (without an actual occultation) the passage of a bright star behind halley's inner coma might allow optical depth measurements or the observation of absorption spectra. The possibility of detecting a returned radar doppler signal from Halley should be investigated. The optimum opportunities for this type of measurement would occur in late November 1985 and early April 1986, when the comet-Earth separation distances reach a minimum of 0.62 and $0.42 \mathrm{AU}$ respectively.

\section{Net Operations}

Assuming the astrometry network will be used to support a Halley flyby mission, the network should consist of at least 6 Northern and 6 Souchern Hemisphere observatories spaced in longitude to avoid weather problems. Each observer should supply a pair of observations per week for the period January - May 1985 and August - September 1985. During periods of Earth-comet close approaches, each observer should supply a pair of observations every 3 days during October 1985 - January 1986 and March 1986.

If the astrometry netwurk will not be required to support a Halley flyby mission, the number of observatories couid easily be cut to 3 northern and 3 southern locations with astrometric positions provided at on $1 \mathrm{y} 20 \%$ of the rate required to support a mission. 
Astrometry net stations could publish their reduced positions as they see fit, but if they are to be of any value in mission support the plates would have to be reduced as soon as they are dry and the positions transmitted immediately to the discipline specialist. The discipline specialist should be an orbital mechanics specialist prepared to imediately update the orbital predictions and cransmit them to the mission control center. This kind of activity may require considerable direct financial support of the astrometric observers, who are not used to working in real-time.

\section{REFERENCES}

1. Bobrovnikoff, N. T., Publ. of the Lick Observatory, 17, 1931.

2. Kormendy, J., Astron. J., 78, 255, 1973.

3. Delseme, A. H., and Rud, D. A., Istron. Astrophys., 28, 1-6, 1973. 
APPENDIX A. EPHEMERIS FOR COMET HALLEY
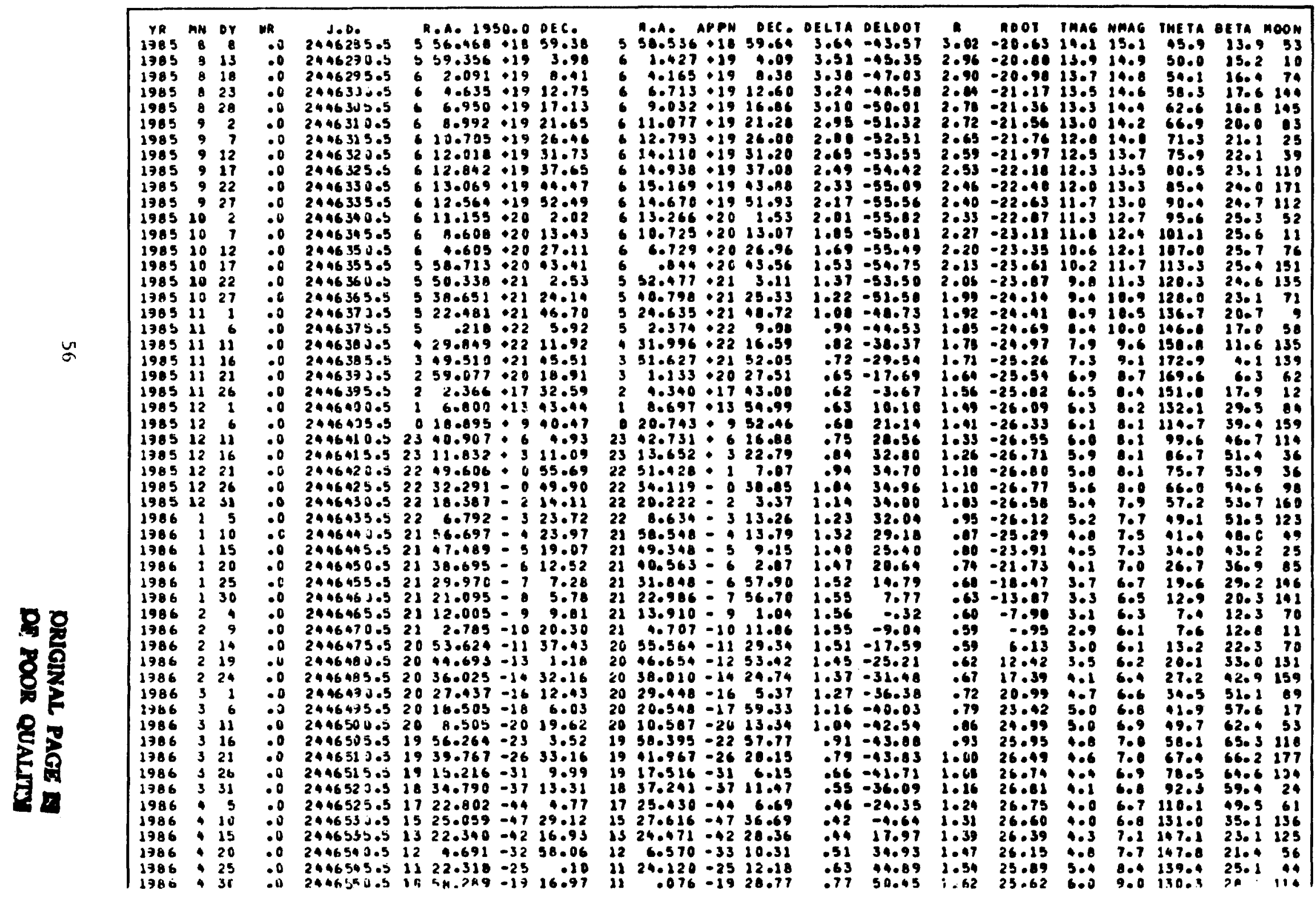


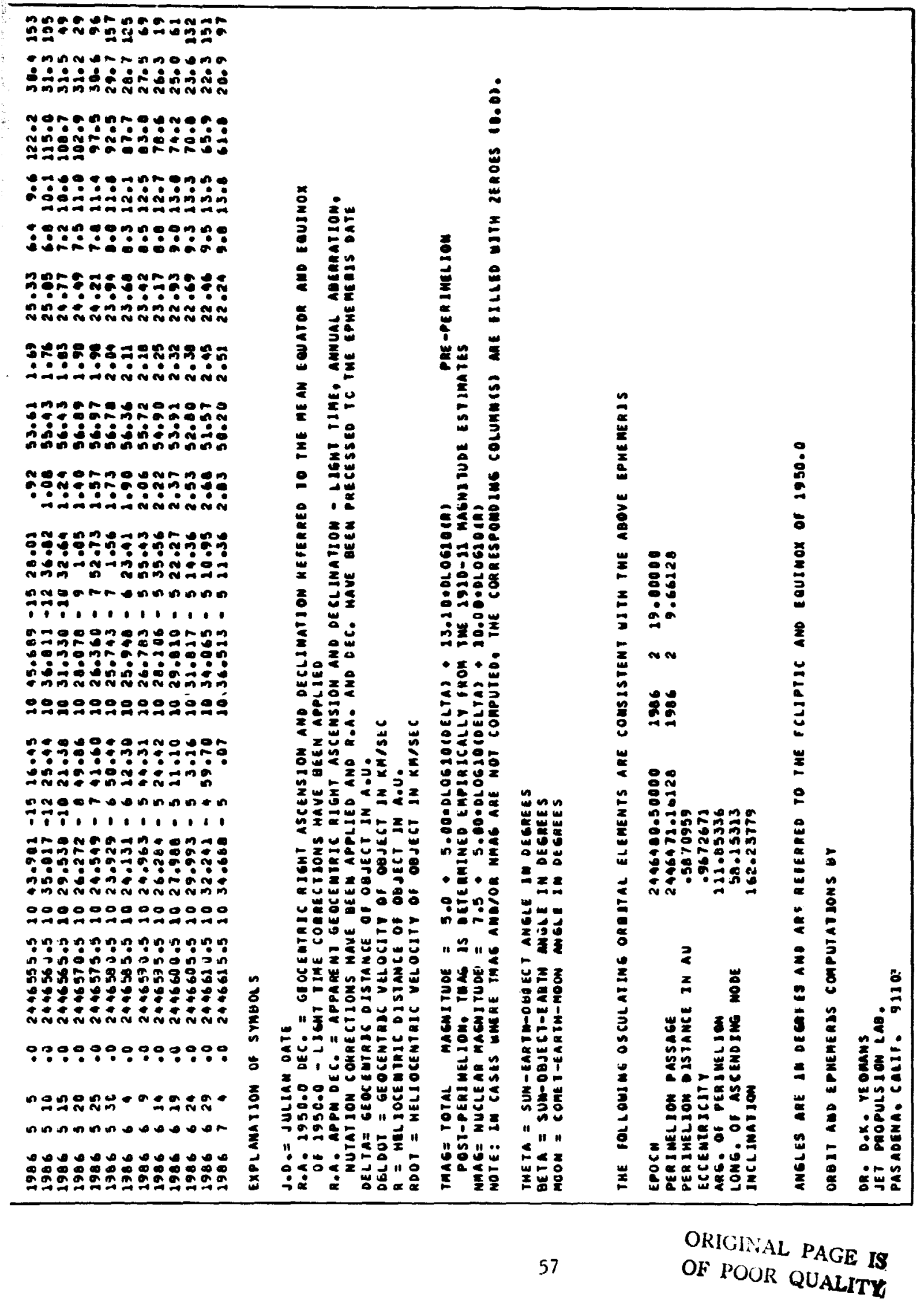


APPENDIX B. OBSERVING CONDITIONS FOR COMET HALLEY

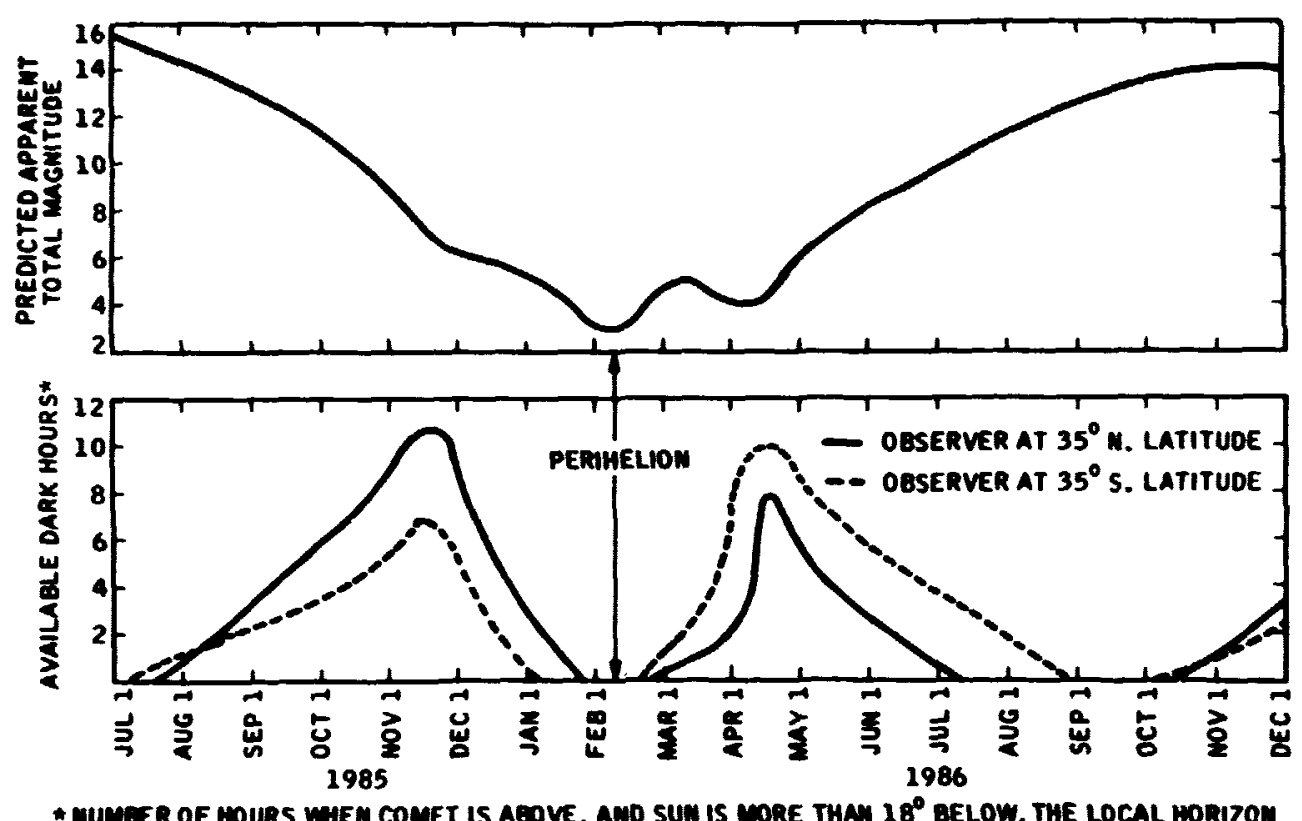


APPENDIX C. CIRCULAR RESPECTING OBSERVATION OF HALLEY'S COMET - 1910

(Courtesy American Astronomical Society) 


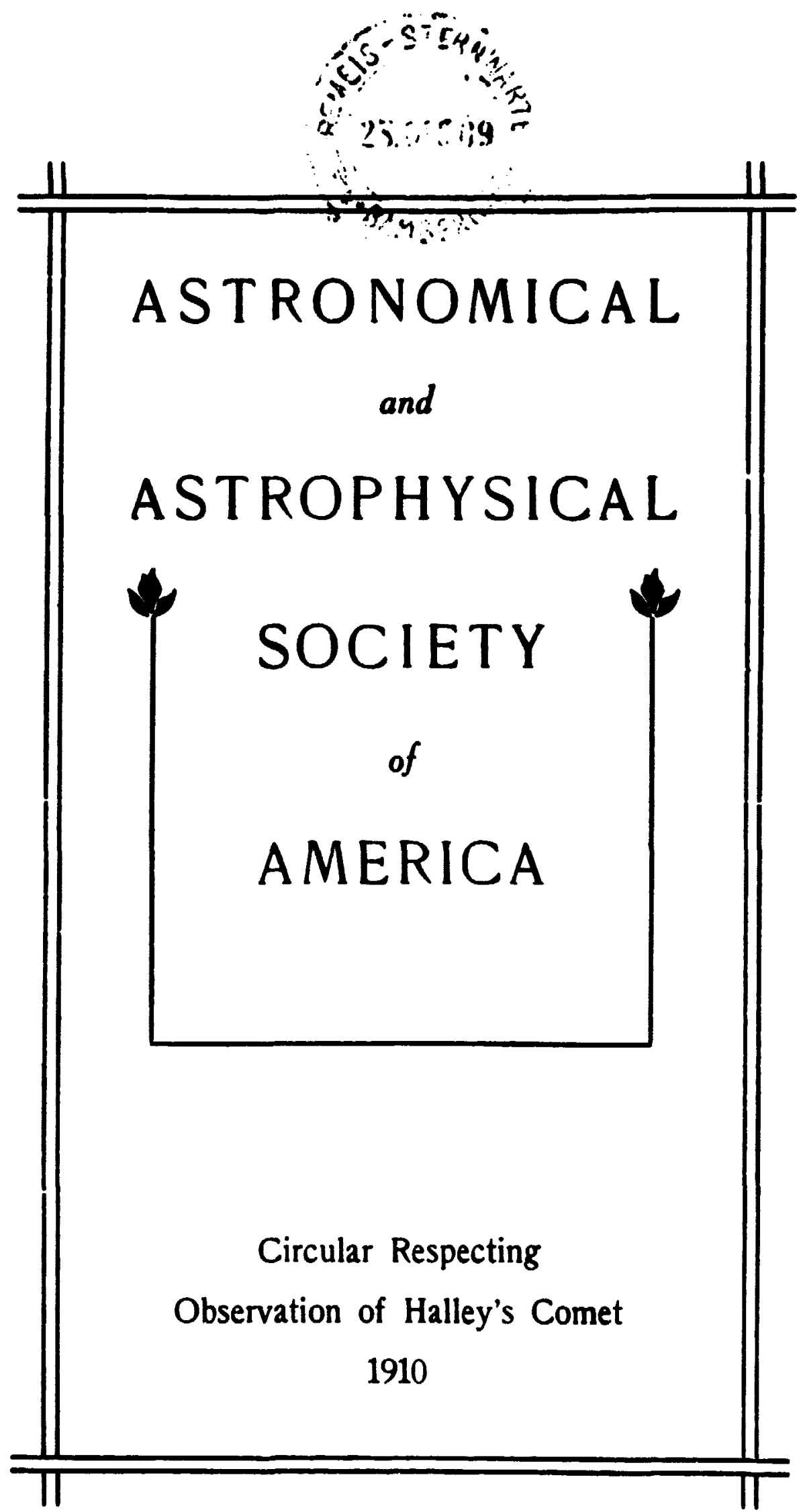




\title{
The Astronomical and Astrophysical Society of America
}

\author{
COMMITTEE ON COMETS \\ QEOROE C. COMSTOCK EDWARD E. BARNARD EDWIN B. FROST \\ CHARLE D. PERRINE EDWARD C. PICKERING
}

Dear Sir:

Madison, Wis., Nov. 10, 1909.

The Comet Committee of the Astronomical and Astrophysical Society of America invites your co-operation in observing $\mathrm{Hal}$ ley's Comet at its impending return, and suhmits for your $\mathrm{c}$.. sideration the following statement with respect to such obser ... tions.

It is desirable that the position of the comet be well obse. i during the entire period of its visibility and it seems probable that extra-meridian observatious will be secured in sufficient number without especial solicitation. In view, however, of possible large perturbations arising from the close approach of the comet to Venus on May 1, and to the earth on May 18, meridian observations are especially desired during the period in which the comet is sufficiently bright for that purpose. An examination of the amount and character of these comet perturbations and their adaptability to a determination of the mass of the planets producing them has been undertaken by Professors Leuschner and Crawford and in case the conditions prove favorable, the meridian determinalions may well be su. plemented by heliometer observations of the positions of the inner planets with a view to a possible determination of the mass of the comet itself.

The close approach of the comet to the earth promises unusual opportunity for a study of the physical conditions that obtain in such a body and, as an indispensabie basis for such study, the Committee recommends a photographic campaign as long and as nearly continuous as possible. The comet's close proximity to the sun's direction at the time of maximum brilliance imposes serious limitations upon this programme and 
widely extended co-operation will be required throughout the whole circuit of the earth, if this ideal of a continuous photographic record is to be even remotely realized.

About one-third of the earth's circumference in longitude is covered by the Pacific Ocean, within which there is known to exist no observatory with proper facilities for celestial photography. To fill this gap, at least partially, the Committee aided by a grant from the National Academy of Seiences, plans to send to the Hawaiian Islands an expadition to photograph the comet during the period of its greatest brilliance.

The ends to be served by these photographs and similar ones obtained elsewhere are conceived by the Committee as follows:

To give a permanent record, as continuous as possible, of the phenomena and changes, (1) in the tail of the comet, with special reference to outgoing masses; (2) in the head and nucleus of the comet, particula:iy as to the formation of envelopes and jets.

The following suggestions as to procedure and precaution in making the photographs have been formulated by Professor Barnard.

\section{Photography of Halley's Comet}

One of the greatest difficulties in photographing the average bright comet is its proximity to the horizon and consequent projection. on a more or less dawn or twilight sky. The effect of chis illuminated background with any considerable exposure is to fog the piate to such an extent as either to ruin it or to prevent a proper development of the image of the comet. A difference of three or four minutes in the duration of exposure when the sky is brightest may make a success or a failure of the piciure. It is impossible to establish fixed rules as to when the exposure should stop or begin, so much will depend upon the condition of the sky, the position of the comet, the kind of lens, the rapidity of the plates, etc. The best rule is that of the judgment of the observer at the time, and this can only be derived from actual experience in the work.

I have examined a large number of negatives of comets taken nnder the above conditions and find that with a quick plate and a 6-inch portrait, lens, on a good sky, the probable result is indicated in the following iable: 


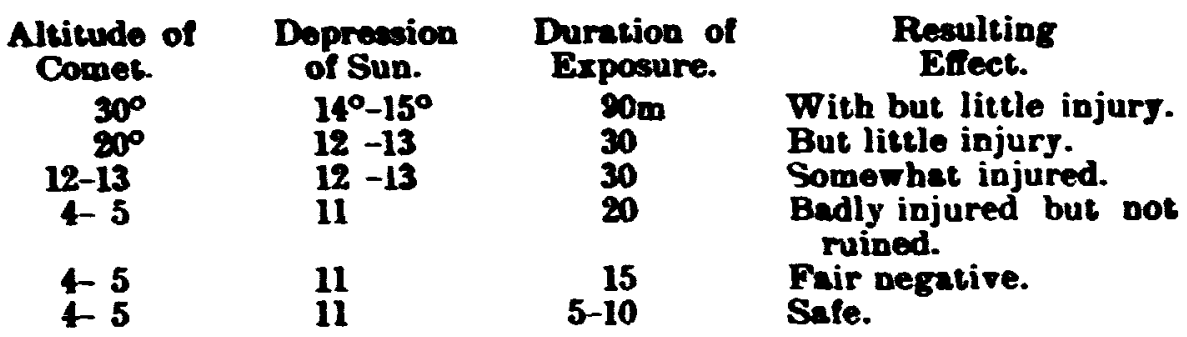

The altitude of the comet and depression of the sun in the above table refer to the beginning of the exposure in the evening and the close in the morning sky. The greatest injury occurs during the first few minutes or the last few as above.

When only moonlight is considered, a half hour's exposure can be made with the moon full, if the comet is not too near it, but the plate will not develop properly. In exceptional cases-a very pure sky-an hour's expcsure will not ruin the plate. With a half moon, not too near the comet, upwards of an hour may be given but with more or less injury to the plate.

A "dew cap" extending as far as will ot cut down the field is very important, not only as a protection from dew, but also to cut off the illumination from the other parts of the sky. It inust be remembered that, on account of its quickness, the small lantern lens will fog a plate much sooner than a larger lens.

Tho plates should be backed with the following to prevent halation. Cook two pounds of white sugar in a saucepan without water nntil nearly in the caramel stage, then add one pound of burnt sienna. Cook a little more (but not to the candy stage) stirring well. Finally, add about, one-haif an ounce of alcohol to each pint of backing as a dryer. This backing will keep indefinite1y. When it is too hard, moisten it with a little water. This is to be applied to the back of the plate as a stiff paste with a broad camel's hair brush, and should be applied just before using. A piece of old newspaper pressed upon this will prevent its being rabbed. The face of the plate should be very carefully dusted with a broad camel's hair brush after it has been placed in the plate-holder. The camera tube should also be frequently wiped out with a damp cloth to avoid dust. Before developing, remcre the backing with moist absorbent cotton. If a little remains on the plate, it will not injure the developer. In removing the backing, be carefui to shield the plate from the dark ro $\mathrm{m}$ light. Do not wet the surface of the plate before pouring 
on the developer as it may canse air bubbles on the film; swab it carefully with absorbent cotton at the beginning of dovelopment. Develop until the plate is almost opaque to the ordinary developing light. Fix for twenty minutes or more, in the ordinary fxing bath (frequently made new), to which has been added a teaspoonful of sodium bisulphite to prevent discoloration.

Lumiere Sigma dry plates are recommended because of their rapidity. Seed 27 Gilt Fdge and Cramer Crown are both beautiful plates, but are not now so rapid as the Sigma.

Hydrochinon developer gives a good strong negative, and for astronomical work is excellent. Rodinal in a weakened formsay 1:60 or 1:70 of water with a longer development will give a soft and more transparent negative, especially suited for showing the details of the head of the comet on large scale photographs.

The doublet, or portrait lens, such as is made in America by the Brashenr Optical Company, and the Alvan Clark Corporation, on account of its wide field, is the best form of instrument for showing the general features of the comet and its tail, and especially for following any outgoing massses that may appear in the tail. One of about six inches $(15 \mathrm{~cm}$.) aperture will be the most generally used because of the expense of such instruments. It should be supplemented by several smaller lenses. A "lantern" lens of one and one-half inches $(4 \mathrm{~cm}$.) aperture and about six inches (15 cm.) focus, made by MeAllister, of New York, is recommended for showing the extent of the tail. The cost of one of these lantern lenses is seven dollars. It gives a good field of twonty to thirty degrees-especially when diaphragmed down to one inch. It is extremely quick for comet work. Its focus should be carefully determined by star trails.

In comet work it is important in all these lenses that the camera should be so adjusted on the mounting with respect to the guiding telescope that the head of the comet can be displaced to one side of the field to secure a greater extension of the tail. $T$ Fo of the small lenses may be so arranged by a mutual adjustment as to cover the full length of the tail, even though it should be fifty degrees long, or more. Although it would thus be in two sections-the head and part of the tail on one plate, and the rest of the tail on the other-there would be no serious 
objection if the whole tail could thns be secured. The large reflectors will be of the utmost importance in dealing with the detail and structure of the head and envelopes as has been rosently shown at Greenwich.

Until something further is known of the spectrum of the comet, it would be unwise to attempt to give any specific directions as to the duration of exposure required with any telescope. Daniel's Comet of 1907, and Morehouse's of 1908 were very different in respect to their photographic activity. The latter was relatively many times more actinic in its light and hence roquired much shorter exposures to show the same strength of tail. This information must come from actual experience with the comet. It would seem, however, that the circumstances of the comet's visibility when brightest will make short exposures necessary. In photographs where a guiding telescope is used, it is best, when possible, to guide with wires on the head or nncleus of the comet itself. In the case of large reflectors, or when the double-slide plate-holder is used, or where the guiding telescope is insufficient to show the comet fairly well, the method of puiding on a star, as used by Dr. Joel Metcalf, would be necessary, and with the proper precautions, will give excellont results. It will be understood that this method requires that the guiding wires be set to move in the path of the comet and be given a motion at short intervals, contrary in directiou, but equal in amount, to that of the comet. When guiding on the head of the comet a careful watch should be kept for any pulsations that may occur in its light (Astrophysical Journal, Dec., 1908, p. 385). If there should be considerable changes the exact time of their occurrence should be recorded.

For a more detailed presentation of the above matter reference may be made to a paper by Professor Barnard, published in Popular Astronomy for December, 1909.

The Committee will be pleased to receive from every astronomer who may co-operate in the matter, copies (glass positives) of his negatives of Halley's Comet, and it will nndertake the comparison and discussion of the material thus collected. 


\section{Spectrosoopic Obervatione of Halley'o Comet}

For spectroscopic observations of the comet the Committeo makes the following suggestions, formulated by Professor Frost: While it may bo possible to make vieual observations of the comet's spectrum with ocular spectroscopes attached to large telescopes, it is likely that most of the photographic records of the spectrum will be obtained by the objective-prism or the slitspectrograph; and reference will made in what follows to the use of these two types of instrument.

These methods of observation are mutually complementary; for the accurate measurement of wave-length, effect of motion in the line of sight, and analysis of structure of lines or bands (if sufficiently sharp) the slit-spectograph has all the advantages; but for study of distribution of elements in different parts of the comet. and for reaching faint details, the prismaticcamera, or objective-prism, with its much greater light-power, is essential. The prismatic-camera may be employed, with a fair possibility of success, when the comet's brightness is ejuivalent to that of a ninth or tenth magnitude star; the slit-spectrograph cannot be hopefully applied before the comet is iwo or three magnitudes brighter. The size and kind of telescoye employed, of course, makes such statements relative rather than absolute, and uncertain at best. Too much here depends upon the comet; if its light is chiefis reflected rather than intrinsic, and the continuous spectrum is predominant, then the comet will have to be much brighter for satisfactory sijectroscopic analysis than if the light is largely intrinsic and concentrated at half a dozen points in the spectrum. Comets showing sudden and marked fluctuation in size or brightness are likely to exhibit changes in the bright band spectrum.

(1) Prismatic-Camera or'Objective-Prism.

The camera should be a doubiet of large angular aperture, 1:4 or 1:5. Usefui observations could be secured if the linear aperture is as small as 4 or 5 inches (10 or $12 \mathrm{~cm}$.). The objective-prism should be of small angle, perhaps $10^{\circ}$ or $15^{\circ}$; if an additional prism is available for the period of the comet's greatest brightness, its angle should be about three times that of the smaller prisin. If the doublet is of comparstively short fccus, as is likely to be the case, it will be found to be quite 
sensitive to focus, and separate exposures will be needed for the blue-violet region and the yellow-green region. Optical parts transparent to ultra-violet would be useful, as there may be some important bands of shorter wave-length than $\lambda 3883$.

A guiding telescope will be necessary to follow the comet's motion, but it need not be of large aperture; it should be adjustable through several degrees, so that different parts of the tail can be made central on the plate. It will be quite a convenience to have the reticle or one wire movable with a divided bead.

It is very desirable to photograph the spectrum of a star before or after the comet, placing the star at such a point on the reticle of the guiding telescope that the stellar lines may serve for comparison.

Professor Pickering suggests that an interesting observation would be to photograph the spectrum of a star when seen through the bright portion of the tail, to see if dark absorption lines conld be detected.

(2) Slit-Spectrograph.

A small spectrograph will be a very useful attachinent to a photographic doubtlet as described above. It necd not be complicated and its disparsion may be small. Apparatus for producing a comparison spectrum is not essential, for a neighboring star of the first type may be brought upon the slit, and its spectrum impressed above and below that of the comet. With one thread of the guiding telescope, or finder, movable, the star can be placed so that its spectrum will fall on the slit as desired. The identity of the cometary band can be thus established better than with the objective-prism. After spectrograms of the comet have thus been obtained it will also be desirable to obtain plates with the slit as narrow as feasible, in order to detect duplicity or complexity of the lines or bands.

Observations with powerful stellar spectographs of the types in use for determining radial velocities will doubtless be made as socn as the comet's brightness permits, but this is likely to be disappointingly late on account of the heavy loss of light in such instruments. The fixed equipment of these instruments will determine their operation by their regular observers. 


\section{Photometry of Halley's Comet}

Photometric and polariscopic obserrations of the comet should certainly be made although they will doubtless occupy a position of subordinate importance. The suggestions of the Committeo in this respect are formulated oy Professor Pickering, as follows:

A great variety of methods may be employed for measuring the light or amount of polarization of the comet. It is suggested that astronomers undertaking this problem should correspond with the Chairman of the Committee, in order that uniform methods may be employed throughout by different observers. The plans proposed below may require modification, according to the instruments availabie.

\section{Light}

A direct estimate, by Argeiander's method, of the entire light of the comet, as seen by the naked eye, or in the smallest telescope with which it is visible, may have a certain value to observers in the future, although large systematic errors are to be expected in such estimates.

It is doubtful if photometric measures of the nucleus of the comet wili have much value, as the results will probably be greatly affected by the coma, and will differ with different photometers and telescopes. If the nucleus be distinctly stellar it may be compared directly with an adjacent star, by means of a double image photometer. The effect of brckground would thus be eliminated. Direct measures with a Zöllner photometer, or similar instrument, would probably have but little value, owing to the effect of the coma. Any series by the same observer with the same instrument would be valuable by itself, and the observations by different persons and different instruments might be subsequently adjusted for systematic differences.

The measures described in Harvard Circular 68 showed that the absorption of light by the tail of Comet $1902 \mathrm{~b}$ was certainly less than a tenth of a magnitude. Similar measures should be made of Halley's Comet. A double image photometer is indispensable for these measures also, to eliminate the effect of background.

The light of different portions of the tril of the conet may 
best be measured by the following method: Take two photographs at the same time with oimilar instruments, using the same kind of plate and developer, and giving equal exposures, taking one in focus, and the other out of focus so that the images of the stars shall appear as circles two or three millimeters in diameter. Make similar enlargements of the two plates, interposing screens of perforated brass. Measures of the opacity of the resulting circular images of different portions of the comet, on one plate, may be compared with the images of stars whose magnitudes are known, as photographed on the other plate. The effect of the light of the sky, or of twilight, may thus be eliminated, and the light of the comet compared with that of a star of known magnitude, spread over a standard area. The two photographs may also be compared directly with a suitable photometer.

Messrs. Barnard and Frost, having also the beuefit of Mr. Parkhurst's opinion, suggest as an alternative aud possibly better method the extra focal use of a single camera. The intensity of the extra-focal cometary image could be reduced to the focal plane as accurately as for the star-images. The relative yalues on different nights would always be correct.

Useful suggestions for the photonetric observations of the comet may be derived from a paper by Dr. Roseniverg upon photometric observations of the Morehouse Comet contained in the Astrophysical Journal, for November, 1909.

\section{Polarization}

The polarization, if any, of the comet's taii may best be studied by photographs taken with a camera having a double image prism placed over the lens. The prism should be turned so that the two images, are perpendicular to the direction of the sun. The two images of an unpolarized object should be alike if the correction for color is the the same for both, otherwise it will be necessary to take a second photograph, turning the prism $180^{\circ}$. It the light is polarized, one image may be fainter than the other, as in in similar photographs of the solar corona. Measures may be made as described in the preceding paragraph.

Bands will doubtless be seen if the comet is examined by means of a Savart's polariscope, or similar instrument, but it 
is in that ease, difficult to distinguish between slight polarization of the comet aud the strong polarization of faint sky light.

\section{Minor Notes}

The following titles may be specified as minor matters not included above but which may under some circumstances become of importance, viz.:

The head of the comet should be carefully examined for traces of phase. Possible disturbances may be found in the comet due to its close approach to Venus on May 1, and to the earth on May 18. A transit of the earth through the comet's tail is possible at or near the latter date and, if such should occur, a meteoric shower should be looked for and observed with reference to a determination of the meteoric particles, their frequence, size, etc. Resultant disturbances of the electric potentiai of the earth's atmosphere are possible and the cooperation of meteorological observers and especially of national weather bureaus is earnestly desired in this connection.

Although the amount of refraction experienced by light in transit through a comet is known to be very small, it seems desirable to make investigation of the matter photographically with long focus telescopes. The position of a sufficiently bright star near the nuclens, or in the brightest part of the edge of the tail, should be referred to a group of more distant ones, and the resulting position of the star compared with that resulting from another plate exposed after the comet has left the star.

The Committee will be pleased to receive suggestions from astronomers as to any of the above lines of investigation, as well as others not here enumerated, and solicits widespread co-operation in utilizing the opportunities presented by the present return of Halley's Comet.

On behalf of the Committee,

GEORGE C. COMSTOCK, Chairman. 


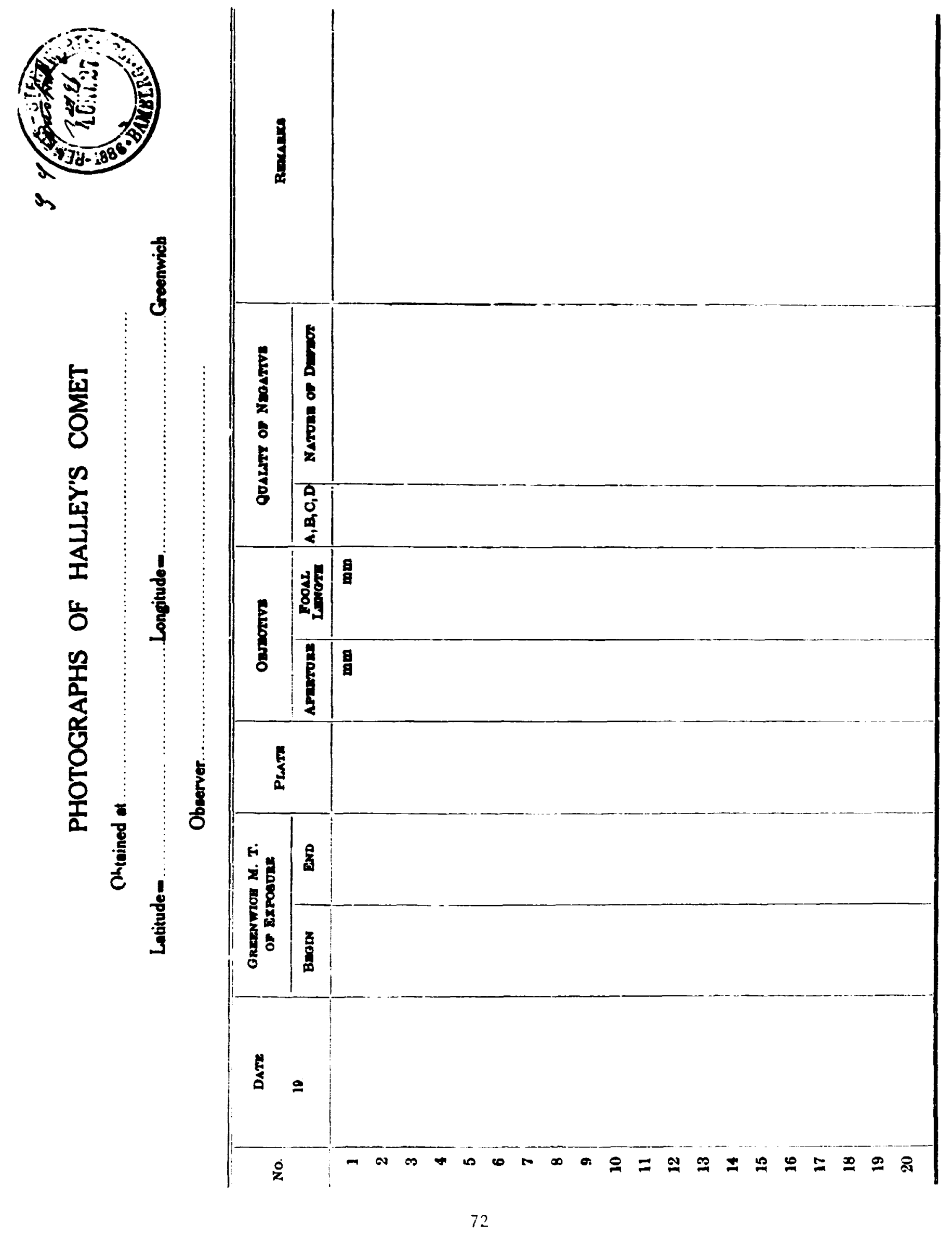

